\title{
Stochastic Reaction-diffusion Equations Driven by Jump Processes
}

\author{
Zdzisław Brzeźniak ${ }^{1}$ - Erika Hausenblas ${ }^{2}$. \\ Paul André Razafimandimby ${ }^{3}$
}

Received: 6 January 2014 / Accepted: 4 September 2017 / Published online: 21 September 2017

(C) The Author(s) 2017. This article is an open access publication

\begin{abstract}
We establish the existence of weak martingale solutions to a class of second order parabolic stochastic partial differential equations. The equations are driven by multiplicative jump type noise, with a non-Lipschitz multiplicative functional. The drift in the equations contains a dissipative nonlinearity of polynomial growth.
\end{abstract}

Keywords Itô integral driven by a Poisson random measure · Stochastic partial differential equations · Lévy processes · Reaction diffusion equations

Mathematics Subject Classification (2010) 60H15 • 60J75 • 60G51

This work was supported by the FWF-Project P17273-N12

Zdzisław Brzeźniak

zdzislaw.brzezniak@york.ac.uk

Erika Hausenblas

erika.hausenblas@unileoben.ac.at

Paul André Razafimandimby

paul.razafimandimby@up.ac.za

1 Department of Mathematics, University of York, Heslington, York YO10 5DD, UK

2 Department of Mathematics and Information Technology, Montanuniversität Leoben, Franz Josef Strasse 18, 8700 Leoben, Austria

3 Department of Mathematics and Applied Mathematics, University of Pretoria, Lynwood Road, Pretoria 0083, South Africa 


\section{Introduction}

The aim of this paper is to study a class of stochastic reaction-diffusion equations driven by a Lévy noise. A motivating example is the following stochastic partial differential equation with a Dirichlet boundary condition

$$
\left\{\begin{aligned}
d u(t, \xi) & =\Delta u(t, \xi) d t+\left[u(t, \xi)-u(t, \xi)^{3}\right] d t \\
& +\frac{\sqrt{|u(t, \xi)|}}{1+\sqrt{|u(t, \xi)|}} d L(t), \quad t>0, \\
u(t, \xi) & =0, \quad \xi \in \partial \mathcal{O}, \\
u(0, \xi) & =u_{0}(\xi), \quad \xi \in \mathcal{O},
\end{aligned}\right.
$$

where $L=\{L(t): t \geq 0\}$ is a real valued Lévy process whose Lévy measure $v$ has finite $p$-moment for some $p \in(1,2]$ and $\mathcal{O} \subset \mathbb{R}^{d}$ is a bounded domain with smooth boundary.

One consequence of our main results is that for every $u_{0} \in C_{0}(\overline{\mathcal{O}})$ there exists a $C_{0}(\overline{\mathcal{O}})$ valued process $u=\{u(t): t \geq 0\}$, which is a martingale solution to problem (1.1). The diffusion coefficient in Eq. 1.1, i.e., the function $g(u)=\sqrt{|u|} /(1+\sqrt{|u|})$, is just a simple example of a bounded and continuous function, the drift term $f(u)=u-u^{3}$ is an example of a dissipative function $f: \mathbb{R} \rightarrow \mathbb{R}$ of polynomial growth, and the Laplace operator $\Delta$ is a special case of a second order operator. Our results allow us to treat equations with more general coefficients than those in the model problem (1.1). In fact we can study stochastic partial differential equations with second order uniformly elliptic dissipative operators with non-constant coefficients and an unbounded nonlinear map as drift term. Moreover, our results are applicable to equations with infinite-dimensional Lévy processes as well as systems with more general initial data, for instance, elements of the Lebesgue or Sobolev spaces $L^{q}(\mathcal{O})$ or $W_{0}^{\gamma, q}(\mathcal{O})$. The details are presented in Sections 4, 5 and 6.

In our paper we adopt the approach used in [10] by the first named author and Gąarek, in which a similar problem but driven by a Wiener process was treated. The major differences of the current work with respect to [10] are as follows. Firstly, the approach [10] heavily depends on the use of the theory of Itô integral in martingale type 2 Banach spaces with respect to a cylindrical Wiener process, while we use an approach which relies on stochastic integration in martingale type $p$ Banach spaces with respect to a Poisson random measure, see [13]. Secondly, the compactness argument in [10] relies on the Hölder continuity of the trajectories of the corresponding stochastic convolution process. However, the trajectories of the stochastic convolution process driven by a Lévy process are not continuous, then the use of the counterpart of the Hölder continuity, i.e. the càdlàg property of the trajectories seems natural to be used. Unfortunately, as many counterexamples have shown, see for instance a recent monograph [65] as well as the recent papers [19] and [11], the trajectories of the stochastic convolution processes driven by a Lévy process may not even be càdlàg in the space in which the Lévy process lives. Hence, this issue has to be handled with special care. Thirdly, we do not use martingale representation theorem, unlike the authors in [10] who used a result by Dettweiler [27]. Instead we use an ad hoc method based on a generalisation of the Skorohod representation theorem, see Theorem C.1. Finally, in order to control certain norms of the approximate solutions, instead of using stopping times as in [10], we apply interpolation methods. Summarizing, this paper contains general results on the existence of martingale solutions to stochastic reaction-diffusion equations with dissipative type coefficients of polynomial growth and with multiplicative Lévy noise. We should also point out that, as a by-product of our results, we are able to fill a gap in the proof of the main result in the article [43]. The gap is related to the Step I, see page 8, in the current paper. 
Our paper confirms an observation that has already been made in earlier papers [13, 42] that the theory of stochastic integration with respect to a Poisson random measure in martingale type $p$ Banach spaces is, to a large extend, analogous to the theory of stochastic integration with respect to a cylindrical Wiener process in martingale type 2 Banach spaces provided the space of $\gamma$-radonifying operators is replaced by the space $L^{p}(v)$, where $v$ is the intensity measure of the Poisson random measure in question. We would also like to point out that the theory of Banach space valued stochastic integrals with respect to a Poisson random measure is richer than the corresponding Gaussian theory, see the recent paper [30] by S. Dirksen. It would be of great interest to develop a theory of inequalities for stochastic convolutions and generalise the paper [13] in the framework of [30].

It is worth mentioning that although SPDEs with unbounded nonlinearity driven by a Lévy process have not been as extensively studied as their Gaussian counterparts, there exists a number of interesting recent publications on the subject. For instance, Truman and $\mathrm{Wu}$ [74, 75], Jacob et al. [61], and Giri and Hausenblas [41] studied equations with Burgers type nonlinearities driven by a Lévy noise. In addition, Dong and Xu in [31] considered the Burgers equation with the compound Poisson noise, thus in fact dealing with a deterministic Burgers' equation on random intervals and random jumps. Some discussion of stochastic Burgers' equations with additive Lévy noise is contained in [19], where it was shown how integrability properties of trajectories of the corresponding Ornstein-Uhlenbeck process play an important role in the existence and uniqueness of solutions. In the recent paper [32] Dong and Xie studied the stochastic Navier-Stokes equations (NSEs) driven by a Poisson random measure with finite intensity measure. Fernando and Sritharan [37] and the first two named authours together with Zhu [17] studied the 2-D stochastic NSEs by means of a local-monotonicity method of Barbu [4]. This method seems to be restricted to the 2-D NSEs and does not require the use of compactness results. In their beautiful monograph [65] Peszat and Zabczyk studied classes of reaction-diffusion equations driven by an additive Lévy process. In this case, the stochastic evolution equations can be transformed into an evolution equation with random coefficients, a method which usually does not work with multiplicative noise. In another recent paper [55] Marinelli and Röckner investigated a certain class of generalized solutions to problems similar to ours. Röckner and Zhang in [67] established the existence and uniqueness of solution to and a large deviation principles for a class of stochastic evolution equations driven by jump processes. Finally, Debussche et al. in $[25,26]$ considered a stochastic Chafee-Infante equation driven by an additive Lévy noise and investigated the dynamics of the equation, for instance, the first exit times from domains of attraction of the stationary solutions of the deterministic equation.

The stochastic PDEs driven by Lévy processes in Banach spaces have not been intensively studied, apart from a few papers by the second named author, like [42, 43], a very recent paper [13] by the first two named authors and [54] by Mandrekar and Rüdiger (who actually studied ordinary stochastic differential equations in martingale type 2 Banach spaces). Martingale solution to SPDEs driven by Lévy processes in Hilbert spaces are not often treated in the literature. Mytnik [60] constructed a weak solution to SPDEs with non-Lipschitz coefficients driven by space-time stable Lévy noise. In [58] Mueller studied non-Lipschitz SPDEs driven by nonnegative stable Lévy noise of index $\alpha \in(0,1)$. Mueller, Mytnik and Stan [59] investigated the heat equation with one-sided time independent stable Lévy noise. One should add that the noise in the paper [58] does not satisfy the hypothesis of the current work.

The current paper is organized as follows. In Section 2 we introduce the notations used later on in the paper and we present the standing hypotheses and essential preliminary facts. Our two main results, i.e Theorems 3.2 and 3.4 are stated in Section 3. In that section we also 
present Theorem 3.5 which could be seen as the reformulation of some of our main results in terms of a Lévy process and not a Poisson random measure. Three examples illustrating the applicability of our results are presented in Sections 4, 5 and 6. To be more precise, SPDEs with dissipative polynomial drift driven by a real-valued $\alpha$-stable tempered Lévy process are treated in Section 4, SPDEs with a bounded drift driven by a space-time Lévy white noise are treated in Section 5 and finally, stochastic reaction-diffusion equations with dissipative polynomial growth drift, driven by a space-time Lévy white noise, are treated in Section 6. In Section 7 we state and prove several preliminary results about the stochastic convolution processes which we believe are interesting in themselves. Sections 8 and 9 are devoted to the proofs of our results. Unfortunately, these proofs are very technical and long and hence their brief outline is presented just after the statement of Theorem 3.5, see page 22. In the appendices we recall some definitions and well-known results in analysis and probability theory. We also prove new results, amongst them a modified version of the Skorohod representation theorem, see Theorem C.1, which are interesting in themselves.

We finish the Introduction by pointing out that the approach presented in this paper (or rather it's earlier arXiv version) has already been taken up and used for the proof of the existence of solutions to Stochastic Navier-Stokes equations and second grade fluids driven by Lévy noise, see [57] and [44], respectively.

Notation 1 By $\mathbb{N}$ we denote the set of natural numbers, i.e., $\mathbb{N}=\{0,1,2, \cdots\}$ and by $\overline{\mathbb{N}}$, respectively $\mathbb{N}^{*}$, we denote the set $\mathbb{N} \cup\{+\infty\}$, respectively $\mathbb{N} \backslash\{0\}$. Whenever we speak about $\mathbb{N}$, respectively $\overline{\mathbb{N}}$-valued measurable functions, we implicitly assume that the set $\mathbb{N}$, respectively $\overline{\mathbb{N}}$, is equipped with the full $\sigma$-field $2^{\mathbb{N}}$, resp. $2^{\overline{\mathbb{N}}}$. By $\mathbb{R}_{+}$we denote the set $[0, \infty)$ of nonnegative real numbers and by $\mathbb{R}_{*}$ the set $\mathbb{R} \backslash\{0\}$. If $X$ is a topological space, then by $\mathcal{B}(X)$ we denote the Borel $\sigma$-field on $X$. By Leb we denote the Lebesgue measure on $\left(\mathbb{R}^{d}, \mathcal{B}\left(\mathbb{R}^{d}\right)\right)$ or $(\mathbb{R}, \mathcal{B}(\mathbb{R}))$. The space of bounded linear operators from a Banach space $Y_{1}$ to a Banach space $Y_{2}$ is denoted by $\mathcal{L}\left(Y_{1}, Y_{2}\right)$. The norm of $A \in \mathcal{L}\left(Y_{1}, Y_{2}\right)$ is denoted by $\|A\|_{\mathcal{L}\left(Y_{1}, Y_{2}\right)}$. If $\mathcal{O} \subset \mathbb{R}^{d}$ is a bounded domain with smooth boundary $\partial \mathcal{O}$, by $C_{0}(\overline{\mathcal{O}})$ we denote the space of real continuous functions on $\overline{\mathcal{O}}$ which vanish on the boundary $\partial \mathcal{O}$.

Suppose that $(Z, \mathcal{Z})$ is a measurable space. By $M(Z)$, respectively $M_{+}(Z)$, we will denote the set of all $\mathbb{R}$, respectively $[0, \infty]$-valued measures on $(Z, \mathcal{Z})$. By $\mathcal{M}(Z)$, respectively $\mathcal{M}_{+}(Z)$, we will denote the $\sigma$-field on $M(Z)$, respectively $M_{+}(Z)$, generated by functions

$$
i_{B}: M(Z) \ni \mu \mapsto \mu(B) \in \mathbb{R},
$$

respectively by functions

$$
i_{B}: M_{+}(Z) \ni \mu \mapsto \mu(B) \in[0, \infty],
$$

for all $B \in \mathcal{Z}$. Similarly, by $M_{I}(Z)$ we will denote the family of all $\overline{\mathbb{N}}$-valued measures on $(Z, \mathcal{Z})$, and by $\mathcal{M}_{I}(Z)$ the $\sigma$-field on $M_{I}(Z)$ generated by functions $i_{B}: M(Z) \ni \mu \mapsto$ $\mu(B) \in \overline{\mathbb{N}}, B \in \mathcal{Z}$.

Finally, by $\mathcal{Z} \otimes \mathcal{B}\left(\mathbb{R}_{+}\right)$we denote the product $\sigma$-field on $Z \times \mathbb{R}_{+}$and by $v \otimes$ Leb we denote the product measure of $v$ and the Lebesgue measure Leb.

For a Banach space $Y$ by $\mathbb{D}([0, T], Y)$ we denote the space of all càdlàg functions $u$ : $[0, T] \rightarrow Y$ which we equip with the $J_{1}$-Skorohod topology, i.e., the finest among all Skorohod topologies. 


\section{Hypotheses, Notations and Preliminaries}

In this section we introduce the notation and hypotheses used throughout the whole paper. Moreover, we present some preliminary results that will be frequently used later on.

\subsection{Analytic Assumptions and Hypotheses}

Let us begin with a list of assumptions. Whenever we use any of them this will be clearly indicated.

Let $E$ be a Banach space and let $A$ be a closed linear densely defined map in $E$. The norm in the space $E$ is denoted by $|\cdot|$ and the norm of any other Banach space $Y$ is denoted by $|\cdot|_{Y}$.

In what follows we will frequently use the following assumptions about the Banach space $E$ and the linear map $A$.

Assumption 1 1(i) Assume that $E$ is a separable UMD and type $p$, for a certain $p \in$ $(1,2]$, Banach space. ${ }^{1}$

1(ii) $A$ is a positive operator in $E$, i.e, a densely defined and closed operator for which there exists $M>0$ such that for $\lambda>0$

$$
\left\|(A+\lambda)^{-1}\right\|_{\mathcal{L}(E)} \leq \frac{M}{1+\lambda} .
$$

1(iii) $-A$ is the infinitesimal generator of an analytic semigroup denoted by $\left(e^{-t A}\right)_{t \geq 0}$ on $E$. We also assume that $A$ has compact resolvent.

1(iv) The semigroup $\left(e^{-t A}\right)_{t \geq 0}$ on $E$ is of contraction type.

1(v) $A$ has the bounded imaginary power (briefly BIP) property, i.e., there exist constants $K>0$ and $\vartheta \in\left[0, \frac{\pi}{2}\right)$ such that

$$
\left\|A^{i s}\right\|_{\mathcal{L}(E)} \leq K e^{\vartheta|s|}, s \in \mathbb{R} .
$$

2(i) There exists a separable Banach space $X$ such that the embedding $E \subset X$ is dense and continuous.

2(ii) The linear map $A$ has a unique extension to $X$. This extension map is still denoted by $A$ and satisfies Assumptions 1(ii), 1(iii) and 1(iv).

Notation 2 For any $\gamma>0$, the completion of $E$ with respect to the norm $\left|A^{-\gamma} \cdot\right|$ will be denoted by $D\left(A^{-\gamma}\right)$. For any $\gamma>0$, the domain of the fractional power operator $A^{\gamma}$ (in $E$ ) will be denoted by $D\left(A^{\gamma}\right)$. With few exceptions we will only speak about fractional powers of the operator $A$ with respect to the space $E$ and not $X$ nor $B$ and hence the notation $A^{\gamma}$ and $D\left(A^{\gamma}\right)$ should be unambiguous. Those few exceptions are when we use notation $D\left(A_{Y}^{\theta}\right)$, for instance in Theorem 3.2. Finally, let us note that since by assumption $A^{-1}$ exists and is bounded (on $E$ ), the fractional powers $A^{-\gamma}, \gamma \geq 0$, are bounded (on $E$ ) too.

Before we proceed further we make the following useful remark.

Remark 2.1 Since $E$ is a separable, UMD and martingale type $p$ Banach space, we infer from [9, Remark 4.2, also Theorem A.4] that for every $\beta \in \mathbb{R}$, the space $B_{0}=D\left(A^{\beta}\right)$ is also

\footnotetext{
${ }^{1}$ It is known that if $E$ has the UMD property and of type $p$ then it is a martingale type $p$ Banach space (see, for instance, [9]).
} 
a UMD, martingale type $p$ Banach space. The linear map $A$ has an extension (or restriction depending on whether $\beta$ is smaller or larger than 0 ) $A_{0}$ (usually denoted by $A$ ) to $B_{0}$ which satisfies Assumptions 1(ii), 1(iii) and 1(v). The operator $-A_{0}$ generates a contraction type semigroup which will still be denoted by $\left\{e^{-t A}\right\}_{t>0}$ on $B_{0}$. Moreover, if $A$ has the BIP property then so has $A_{0}$.

One of the consequences of the BIP property in Assumption 1(v) is that the fractional domains of the operator $A$ are equal to the complex interpolation space of an appropriate order between $D(A)$ and $E$, see e.g. [72].

Finally, if a linear operator $A$ on a Banach space $E$ is positive, then $-A$ is the infinitesimal generator of a $C_{0}$-semigroup in $E$, see for instance [10, Remark 2.1].

If $E$ is a separable Banach space and $q \in[1, \infty)$, we denote by $L^{q}(0, T ; E)$ (see, for instance, [29]) the Lebesgue space consisting of (equivalence classes of) Lebesgue measurable functions $u:[0, T] \rightarrow E$ such that

$$
\|u\|_{L^{q}(0, T ; E)}:=\left(\int_{0}^{T}|u(s)|^{q} d s\right)^{\frac{1}{q}}
$$

is finite. The Besov-Slobodetskii space $W^{\alpha, q}(0, T ; E)$, where $\alpha \in(0,1)$, consists of all $u \in L^{q}(0, T ; E)$ such that the seminorm

$$
|u|_{W^{\alpha, q}(0, T ; E)}:=\left(\int_{0}^{T} \int_{0}^{T} \frac{|u(t)-u(s)|^{q}}{|t-s|^{1+\alpha q}} d s d t\right)^{\frac{1}{q}}
$$

is finite. The space $L^{q}(0, T ; E)$ and $W^{\alpha, q}(0, T ; E)$ equipped with the norms (2.2) and, respectively,

$$
\|u\|_{W^{\alpha, q}(0, T ; E)}:=\left(\int_{0}^{T}|u(s)|^{q} d s+\int_{0}^{T} \int_{0}^{T} \frac{|u(t)-u(s)|^{q}}{|t-s|^{1+\alpha q}} d s d t\right)^{\frac{1}{q}}
$$

are separable Banach spaces.

We will denote by $C([0, T] ; E)$ the Banach space of all $E$-valued continuous functions defined on the interval $[0, T]$ equipped with the supremum norm. Similarly, if $k \in \mathbb{N}^{*}$, then by $C^{k}([0, T] ; E)$ we will denote the Banach space of all $E$-valued functions of $C^{k}$-class. We will denote by $C^{\beta}([0, T] ; E)$, for $\beta \in(0,1)$, a (non-separable) Banach space of all functions $u \in C([0, T] ; E)$ such that

$$
\|u\|_{C^{\beta}([0, T] ; E)}:=\sup _{0 \leq t \leq T}|u(t)|+\sup _{0 \leq s<t \leq T} \frac{|u(t)-u(s)|}{|t-s|^{\beta}}<\infty .
$$

We will also denote by $H^{1, q}(0, T ; E)$ the space of functions $u \in L^{q}(0, T ; E)$ with weak derivatives $B u:=u^{\prime} \in L^{q}(0, T ; E)$. Endowed with the graph norm of $B$, the space $H^{1, q}(0, T ; E)$ is a Banach space. By $H_{0}^{1, q}(0, T ; E)$ we denote the subspace of $H^{1, q}(0, T ; E)$ consisting of all $u \in H^{1, q}(0, T ; E)$ such that $u(0)=0$.

Now we introduce an important operator $\Lambda=\Lambda_{T}$ which will play a crucial role in our analysis. We start by setting

$$
\begin{aligned}
B u & =u^{\prime}, \quad u \in D(B), \\
D(B) & =H_{0}^{1, q}(0, T ; E) .
\end{aligned}
$$

The space $D(B)$ is a Banach space when equipped with the graph norm

$$
|u|_{D(B)}:=|u|_{L^{q}(0, T ; E)}+\left|u^{\prime}\right|_{L^{q}(0, T ; E)}, u \in D(B) .
$$


Define also a linear operator $\mathcal{A}$ by the formula

$$
\begin{aligned}
D(\mathcal{A}) & =\left\{u \in L^{q}(0, T ; E): A u(\cdot) \in L^{q}(0, T ; E)\right\}, \\
\mathcal{A} u & :=\{[0, T] \ni t \mapsto A(u(t)) \in E\} .
\end{aligned}
$$

The domain $D(\mathcal{A})$ of $\mathcal{A}$ is a Banach space with norm

$$
|u|_{D(\mathcal{A})}:=\left|u^{\prime}\right|_{L^{q}(0, T ; E)}+|A u|_{L^{q}(0, T ; E)}, u \in D(\mathcal{A}) .
$$

Let us note that if $A+\kappa I, \kappa \geq 0$, satisfies parts 1(ii), 1(iii) and 1(v) of Assumption 1, then $\mathcal{A}+\kappa I$ satisfies them as well, see Dore and Venni [33].

Finally, we define the operator $\Lambda$ by

$$
\begin{aligned}
\Lambda & :=B+\mathcal{A}, \\
D(\Lambda) & :=D(B) \cap D(\mathcal{A}) .
\end{aligned}
$$

The domain $D(\Lambda)$ is endowed with the graph norm., i.e,

$$
|u|_{D(\Lambda)}=|u|_{D(B)}+|u|_{D(\mathcal{A})}, u \in D(\Lambda) .
$$

We recall that although $\Lambda$ is the sum of the closed operators $\mathcal{A}$ and $B$, it is not necessarily a closed operator. However, if $E$ is an UMD Banach space and $A+\kappa I$, for some $\kappa \geq 0$, satisfies parts 1(ii), 1(iii) and 1(v) of Assumption 1 then, since $\Lambda=B-\kappa I+\mathcal{A}+\kappa I$, by Dore and Venni [33], see also Giga and Sohr [39], $\Lambda$ is a positive operator. In particular, $\Lambda$ has a bounded inverse. Consequently, one can define the fractional powers $\Lambda^{-\alpha}, \alpha \geq 0$. In particular, for $\alpha \in[0,1], \Lambda^{-\alpha}$ is a bounded linear map in $L^{q}(0, T ; E)$, and for $\alpha \in(0,1)$,

$$
\left(\Lambda^{-\alpha} f\right)(t)=\frac{1}{\Gamma(\alpha)} \int_{0}^{t}(t-s)^{\alpha-1} e^{-(t-s) A} f(s) d s,
$$

for any $t \in(0, T), f \in L^{q}(0, T ; E)$. Under the parts 1(i)-1(v) of Assumption 1, the parabolic operator $\Lambda$ and its fractional powers $\Lambda^{-\alpha}, \alpha \in[0,1]$, enjoy several nice properties for which we refer the reader to [10]. The properties of $\Lambda^{-\alpha}$ which are the most relevant to our study are summarized in the following lemma whose proof can be found in [10, Theorem 2.6 and Corollary 2.8].

Lemma 2.2 Assume that $E$ is an UMD Banach space and an operator A satisfying Assumptions 1(ii)-1(iii) is such that $A+\kappa I$, for some $\kappa \geq 0$, satisfies Assumption 1(v). We also suppose that $(A+\kappa I)^{-1}$ is a compact operator in $E$. Let $\alpha, \beta, \delta$ be nonnegative numbers satisfying

$$
0 \leq \beta+\delta<\alpha-\frac{1}{q}
$$

Then, $\Lambda^{-\alpha}$ is a compact linear map from $L^{q}(0, T ; E)$ into $C^{\beta}\left([0, T] ; D\left(A^{\delta}\right)\right)$. In particular, if $\alpha>\frac{1}{q}$, then $\Lambda^{-\alpha}$ is a compact map from $L^{q}(0, T ; E)$ into $C([0, T] ; E)$.

\subsection{Stochastic Preliminaries}

The aim of this subsection is to introduce some additional probabilistic notation. We also present some basic results about stochastic integration with respect to compensated Poisson random measure.

Assumption 2 Let us assume that $(Z, \mathcal{Z})$ is a measurable space, $v \in M_{+}(Z)$, i.e., $v$ is a nonnegative measure on $(Z, \mathcal{Z})$. 
We assume that $\mathfrak{P}=(\Omega, \mathcal{F}, \mathbb{F}, \mathbb{P})$, where $\mathbb{F}=\left(\mathcal{F}_{t}\right)_{t \geq 0}$, is a filtered probability space satisfying the so called usual conditions, i.e.,

(i) $\mathbb{P}$ is complete on $(\Omega, \mathcal{F})$,

(ii) for each $t \in \mathbb{R}_{+}, \mathcal{F}_{t}$ contains all $(\mathcal{F}, \mathbb{P})$-null sets,

(iii) the filtration $\mathbb{F}$ is right-continuous.

Let us start by recalling the following definition which is taken from [45, Definition I.8.1].

Definition 2.3 In the framework of Assumption 2, a time-homogeneous Poisson random measure on $(Z, \mathcal{Z})$ over $\mathfrak{P}$ with the intensity measure $v \otimes$ Leb, is a random variable

$$
\eta:(\Omega, \mathcal{F}) \rightarrow\left(M_{I}\left(Z \times \mathbb{R}_{+}\right), \mathcal{M}_{I}\left(Z \times \mathbb{R}_{+}\right)\right)
$$

satisfying the following conditions

(a) for each $U \in \mathcal{Z} \otimes \mathcal{B}\left(\mathbb{R}_{+}\right), \eta(U):=i_{U} \circ \eta: \Omega \rightarrow \overline{\mathbb{N}}$ is a Poisson random variable with parameter ${ }^{2} \mathbb{E} \eta(U)$;

(b) $\quad \eta$ is independently scattered, i.e., if the sets $U_{j} \in \mathcal{Z} \otimes \mathcal{B}\left(\mathbb{R}_{+}\right), j=1, \cdots, n$ are pairwise disjoint, then the random variables $\eta\left(U_{j}\right), j=1, \cdots, n$ are pairwise independent;

(c) for all $U \in \mathcal{Z}$ and $I \in \mathcal{B}\left(\mathbb{R}_{+}\right)$,

$$
\mathbb{E}[\eta(U \times I)]=v \otimes \operatorname{Leb}(U \times I)=v(U) \operatorname{Leb}(I) ;
$$

(d) for each $U \in \mathcal{Z}$, the $\overline{\mathbb{N}}$-valued process

$$
(0, \infty) \times \Omega \ni(t, \omega) \mapsto \eta(\omega)(U \times(0, t])
$$

is $\mathbb{F}$-adapted and its increments are independent of the past, i.e., the increment between times $t$ and $s, t>s>0$, are independent form the $\sigma$-field $\mathcal{F}_{s}$.

If $\eta$ is a time-homogenous Poisson random measure as above, then by $\tilde{\eta}$ we will denote the corresponding compensated Poisson random measure defined by

$\tilde{\eta}(U \times I)=\eta(U \times I)-\mathbb{E}(\eta(U \times I))=\eta(U \times I)-v(U) \operatorname{Leb}(I), \quad U \in \mathcal{Z}, I \in \mathcal{B}\left(\mathbb{R}_{+}\right)$, with the convention that $\infty-\infty=0$.

We proceed to the definition of functional spaces that we need throughout the paper. Suppose that $Y$ is a separable Banach space. We denote by $L^{q}(Z, v ; Y), q \in[1, \infty)$, the space of all (equivalence classes of) measurable functions $\xi:(Z, \mathcal{Z}) \rightarrow(Y, \mathcal{B}(Y))$ such that

$$
|\xi|_{L^{q}(Z, v ; Y)}:=\left(\int_{Z}|\xi|_{Y}^{q} v(d z)\right)^{\frac{1}{q}}<\infty .
$$

Similarly, we define the space $L^{p}(\Omega ; Y)$ and $L^{q}\left(\Omega_{T} ; Y\right)$, where $\Omega_{T}=[0, T] \times \Omega$, see [29]. In the latter case, we consider the product $\sigma$-field $\mathcal{B}([0, T]) \times \mathcal{F}$. By $L^{0}(\Omega ; Y)$ we denote the set of measurable functions from $(\Omega, \mathcal{F})$ to $Y$.

For $T \in(0, \infty]$ let $\mathcal{N}(0, T ; Y)$ be the space of (equivalence classes of) progressively measurable processes $\xi:[0, T) \times \Omega \rightarrow Y$.

\footnotetext{
${ }^{2}$ If $\mathbb{E} \eta(U)=\infty$, then obviously $\eta(U)=\infty$ a.s..
} 
For $q \in(1, \infty)$ we set

$$
\begin{aligned}
& \mathcal{N}^{q}(0, T ; Y)=\left\{\xi \in \mathcal{N}(0, T ; Y): \int_{0}^{T}|\xi(t)|_{Y}^{q} d t<\infty \mathbb{P} \text {-a.s. }\right\}, \\
& \mathcal{M}^{q}(0, T ; Y)=\left\{\xi \in \mathcal{N}(0, T ; Y): \mathbb{E} \int_{0}^{T}|\xi(t)|_{Y}^{q} d t<\infty\right\} .
\end{aligned}
$$

Let $\mathcal{N}_{\text {step }}(0, T ; Y)$ be the space of all $\xi \in \mathcal{N}(0, T ; Y)$ for which there exists a partition $0=t_{0}<t_{1}<\cdots<t_{n}<T$ such that for $k \in\{1, \cdots, n\}$, for $t \in\left(t_{k-1}, t_{k}\right], \xi(t)=$ $\xi\left(t_{k}\right)$ is $\mathcal{F}_{t_{k-1}}$-measurable and $\xi(t)=0$ for $t \in\left(t_{n}, b\right)$. We put $\mathcal{M}_{\text {step }}^{q}=\mathcal{M}^{q} \cap \mathcal{N}_{\text {step. }}$. It can be easily shown that $\mathcal{M}^{q}(0, T ; Y)$ is a closed subspace of $L^{q}([0, T) \times \Omega ; Y) \cong$ $L^{q}\left([0, T) ; L^{q}(\Omega ; Y)\right)$.

Now for $\xi \in \mathcal{M}_{\text {step }}^{p}\left(0, T ; L^{p}(Z, \nu ; E)\right)$ we set

$$
\tilde{I}(\xi)=\sum_{j=1}^{n} \int_{Z} \xi\left(t_{j}, z\right) \tilde{\eta}\left(d z,\left(t_{j-1}, t_{j}\right]\right) .
$$

It is shown in [13] that if $E$ is a Banach space of martingale type $p \in(1,2]$, then $\tilde{I}$ is a bounded linear map from $\mathcal{M}_{\text {step }}^{p}\left(0, T ; L^{p}(Z, v ; E)\right.$ ) (with respect to the norm inherited from $\left.\mathcal{M}^{p}\left(0, T ; L^{p}(Z, v ; E)\right)\right)$ to $L^{p}(\Omega, E)$. In particular, there exists a positive constant $C>0$ which depends only on $p$ and $E$ such that

$$
\mathbb{E}|\tilde{I}(\xi)|^{p} \leq C \int_{0}^{T} \mathbb{E} \int_{Z}|\xi(t, z)|^{p} v(d z) d t
$$

and $\mathbb{E} \tilde{I}(\xi)=0$ for any $\xi \in \mathcal{M}_{\text {step }}^{p}\left(0, T ; L^{p}(Z, v ; E)\right)$. From these facts, we can define by Eq. 2.12 the stochastic integral of a process $\xi \in \mathcal{M}_{\text {step }}^{p}\left(0, T ; L^{p}(Z, v ; E)\right)$ with respect to the compound random Poisson measure $\tilde{\eta}$. The extension of this integral to $\mathcal{M}^{p}\left(0, T, L^{p}(Z, v, H)\right)$ is possible, thanks to the density of $\mathcal{M}_{\text {step }}^{p}\left(0, T, L^{p}(Z, v ; E)\right)$ in the space $\mathcal{M}^{p}\left(0, T, L^{p}(Z, v ; E)\right)$. More precisely, we recall the following result whose proof can be found in [13, Theorem C.1].

Theorem 2.4 Assume that $p \in(1,2]$ and $E$ is a martingale type $p$ Banach space. Then there exists a unique bounded linear operator

$$
I: \mathcal{M}^{p}\left(0, T, L^{p}(Z, v ; E)\right) \rightarrow L^{p}(\Omega, \mathcal{F}, E),
$$

such that for $\xi \in \mathcal{M}_{\text {step }}^{p}\left(0, T, L^{p}(Z, v ; E)\right)$ we have $I(\xi)=\tilde{I}(\xi)$. In particular, there exists a positive constant $C$ which depends only on $E$ and $p$ such that

$$
\mathbb{E}|I(\xi)|^{p} \leq C \mathbb{E} \int_{0}^{\infty} \int_{Z}|\xi(t, x)|^{p} v(d x) d t,
$$

for every $\xi \in \mathcal{M}^{p}\left(0, T, L^{p}(Z, v ; E)\right)$. Moreover, if $\xi \in \mathcal{M}^{p}\left(0, T, L^{p}(Z, v ; E)\right)$, then the process $I\left(1_{[0, t]} \xi\right), t \geq 0$, where

$$
\left[1_{[0, t]} \xi\right](r, x ; \omega):=1_{[0, t]}(r) \xi(r, x, \omega), t \geq 0, r \in \mathbb{R}_{+}, x \in Z \text { and } \omega \in \Omega,
$$

is an E-valued p-integrable martingale.

The above construction is done in the spirit of Métivier, see [56, Exercise 9, p. 195], see also [13] for further details. As pointed out to the authours by the referee, the construction 
of the Itô stochastic integral can also be done by defining the stochastic integral first for predictable integrands, and then extending the stochastic integral to progressively measurable integrands using the fact that the dual predictable projection of a Poisson random measure, see Theorem II.1.8 in [46], is absolutely continuous with respect to the Lebesgue measure (with respect to the "time" variable). See also [3] for a recent use of this classical notion.

As usual, we put

$$
\int_{0}^{t} \int_{Z} \xi(s, z) \tilde{\eta}(d z, d s):=I\left(1_{[0, t]} \xi\right), \quad t \in[0, T] .
$$

Let us state the following useful result which can be proved using the argument of [15, Proof of Theorem 3.4].

Proposition 2.5 Assume that $(Z, \mathcal{Z})$ is a measurable space, $v$ is a nonnegative measure on $(Z, \mathcal{Z})$ and $\mathfrak{P}=\left(\Omega, \mathcal{F},\left(\mathcal{F}_{t}\right)_{t \geq 0}, \mathbb{P}\right)$ is a filtered probability space. Assume also that $\eta_{1}$ and $\eta_{2}$ are two time-homogeneous Poisson random measures on $(Z, \mathcal{Z})$ over $\mathfrak{P}$, both with the same intensity measures $v \otimes$ Leb. Assume that $p \in(1,2], E$ is a martingale type $p$ Banach space and $\xi \in \mathcal{M}^{p}\left(0, T, L^{p}(Z, v ; E)\right)$.

If $\mathbb{P}$-a.s. $\eta_{1}=\eta_{2}$ on $M_{I}(Z \times[0, T])$, then for any $t \in[0, T], \mathbb{P}$-a.s.

$$
\int_{0}^{t} \xi(s, z) \tilde{\eta}_{1}(d z, d s)=\int_{0}^{t} \xi(s, z) \tilde{\eta}_{2}(d z, d s) .
$$

We close this section with the following maximal inequality whose proof can be found in [13, Corollary C2].

Theorem 2.6 Assume that $1 \leq q \leq p<2$ and $E$ is a martingale type $p$ Banach space. Then, there exists a constant $C>0$ such that for any $T>0$ and any process $\xi \in \mathcal{M}^{p}\left(0, T, L^{p}(S, v ; E)\right)$ we have

$$
\mathbb{E} \sup _{t \in[0, T]}\left|\int_{0}^{t} \int_{S} \xi(r, x) \tilde{\eta}(d x, d r)\right|^{q} \leq C \mathbb{E}\left(\int_{0}^{T} \int_{S}|\xi(r, x)|^{p} v(d x) d r\right)^{q / p} .
$$

\subsection{Lévy Processes and Poisson Random Measures}

The subject of this section is to give a short account on the correspondence between Lévy processes and Poisson random measures. Namely, given a Lévy process on a Banach space, one can construct a corresponding Poisson random measure. Conversely, given a Poisson random measure on a Banach space, one may find the corresponding Lévy process. To illustrate this fact, let us first recall the definition of a Lévy process.

Definition 2.7 Let $Z$ be a Banach space. An $Z$-valued stochastic process $L=\{L(t): t \geq$ $0\}$ over a probability space $(\Omega, \mathcal{F}, \mathbb{P})$ is called an $Z$-valued Lévy process iff the following conditions are satisfied.

(i) $L_{0}=0$ a.s.;

(ii) for all $n \in \mathbb{N}^{*}$ and $0 \leq t_{0}<t_{1}<\cdots t_{n}$, the random variables $L\left(t_{0}\right), L\left(t_{1}\right)-L\left(t_{0}\right)$, $\ldots, L\left(t_{n}\right)-L\left(t_{n-1}\right)$ are independent;

(iii) for all $0 \leq s, t$, the laws of $L(t+s)-L(s)$ and $L(t)$ are equal;

(iv) the process $L$ is stochastically continuous;

(v) the trajectories of $L$ are a.s. $Z$-valued cádlág. 
If $\mathbb{F}=\left\{\mathcal{F}_{t}\right\}_{t \geq 0}$ is a filtration on $\mathcal{F}$, we say that an $Z$-valued stochastic process $L=\{L(t)$ : $t \geq 0\}$ is a Lévy process over a filtered probability space $(\Omega, \mathcal{F}, \mathbb{F}, \mathbb{P})$ iff it is $\mathbb{F}$-adapted, satisfies conditions (i), (iii-v), and

(ii)' for all $0 \leq s<t$, the increment $L(t)-L(s)$ is independent of $\mathcal{F}_{s}$.

In order to discuss Lévy processes in more detail we need to recall a definition of a Lévy measure.

Definition 2.8 (Linde [51, section 5.4]) A $\sigma$-finite Borel measure $\lambda$ on a separable Banach space $Z$ is called a Lévy measure on $Z$ iff its symmetric part $\lambda+\lambda^{-}$, where $\lambda^{-}(A):=$ $\lambda(-A), A \in \mathcal{B}(Z)$, is a symmetric Lévy measure, i.e.,

(i) $\lambda+\lambda^{-}(\{0\})=0$, and

(ii) the function, with $Z^{\prime}$ being the dual of $Z$,

$$
Z^{\prime} \ni a \mapsto \exp \left(\int_{Z}(\cos \langle x, a\rangle-1) \mu(d x)\right)
$$

is a characteristic function of a Borel $^{3}$ probability measure on $Z$.

The class of all Lévy measures on $Z$ is denoted by $\mathscr{L}(Z)$.

For the readers convenience let us now list a few basic properties of Lévy measures, see [51], Theorem 5.4 .8 (i,ii) and Proposition 5.4 .5 (i,ii,iv). First let us recall a useful notation $[51$, p. 68]:

$$
K(x, a):=e^{i\langle x, a\rangle}-1-i\langle x, a\rangle 1_{U_{1}}(x), \quad x \in Z, a \in Z^{\prime},
$$

where $\langle x, a\rangle$ is a shortcut notation for the duality pairing ${ }_{Z}\langle x, a\rangle_{Z^{\prime}}$ and, for $r>0, U_{r}$ denotes the closed ball with radius $r$ and centered at 0 in $Z$.

Proposition 2.9 Suppose that $\lambda$ is a $\sigma$-finite Borel measure on a separable Banach space Z. Consider the following conditions.

(i') $\lambda(\{0\})=0$;

(i) $\lambda$ is a Lévy measure on $Z$,

(ii) For every $a \in Z^{\prime}, \int_{Z}|K(x, a)| d \lambda(x)<\infty$ and the map

$$
Z^{\prime} \ni a \mapsto e^{\int_{Z} K(x, a) d \lambda(x)} \in \mathbb{C}
$$

is a characteristic function of a Borel probability measure, denoted by $e_{s}(\lambda)$, on $Z$;

(ii') for every $\delta>0, \lambda\left(Z \backslash U_{\delta}\right)<\infty$;

(iv') $\sup \left\{\left.\left.\int_{U_{1}}\right|_{Z}\langle z, a\rangle_{Z^{\prime}}\right|^{2} v(d z): a \in Z^{\prime},|a| \leq 1\right\}<\infty$.

Then we have the following implication: $(i) \Longrightarrow\left(i^{\prime}\right) \vee\left(i i^{\prime}\right) \vee\left(i v^{\prime}\right)$. Moreover, if $\left(i^{\prime}\right)$ holds, then $(i) \Longleftrightarrow(i i)$.

Suppose now that $L=\{L(t): t \geq 0\}$ is an $Z$-valued Lévy process over a probability space $(\Omega, \mathcal{F}, \mathbb{P})$, where $Z$ is a separable Banach space. Then for each $t \geq 0$ the measure $\mu_{t}$ being the law of the $Z$-valued random variable $L(t)$ is infinitely divisible and hence, see

\footnotetext{
${ }^{3}$ Since we assume that $Z$ is a separable Banach space, then every Borel probability measure on $Z$ is Radon, see [62, Theorem II.3.2].
} 
[51, Theorem 5.7.3], there exist a Lévy measure $v_{t}$ on $Z$, a Gaussian measure $\rho_{t}$ on $Z$ and a vector $x_{t} \in Z$ such that

$$
\mu_{t}=e_{s}\left(v_{t}\right) * \rho_{t} * \delta_{x_{t}} .
$$

A proof of a finite dimensional version of this result can be found in [70, Theorem 8.1].

From now on we will assume that the process $L$ is purely non-Gaussian, i.e., that $\rho_{t}=\delta_{0}$ for all $t \geq 0$, see [70, Definition 8.2]. We also assume that $x_{t}=0$ for all $t \geq 0$. Thus, see [51, Remark p.84],

$$
\hat{\mu}_{t}(a)=e^{\int_{Z} K(x, a) d v_{t}(x)}, \quad a \in Z^{\prime},
$$

i.e.,

$$
\mathbb{E} e^{i\langle L(t), a\rangle}=e^{\int_{Z} K(x, a) d v_{t}(x)}, \quad a \in Z^{\prime} .
$$

Because $L$ is a Lévy process, and not simply an additive process, the measures $\left(\mu_{t}\right)_{t \geq 0}$ form a convolution semigroup and therefore, the measure $v_{t}$ is equal to $t v$, where $v=v_{1}$. A purely non-Gaussian $Z$-valued Lévy process with $x_{t}=0$ for all $t \geq 0$ satisfying (2.18) will be called a Lévy process with generating triplets $\{0, t v, 0\}$.

The following theorem is a generalisation of (a version of) Theorem 19.2 from [70]. The result below is an infinite dimensional generalisation of a summary of the first two steps of the proof of [70, Theorem 19.2]. This generalisation is possible because of three important inequalities used by Sato are also true in general separable Banach spaces. Firstly, Lemma 20.2 from [70] is true in Banach spaces, see for instance Prop 1.1.1 on p. 15 in the monograph [50] by Kwapień and Woyczyński. Secondly, Remark 20.3 in [70] is just Remark 1.1.1 p. 17 in [50]. Finally, Lemma 20.5 in [70] is Theorem 1 on p. 29 in the Kahane's book [49].

Theorem 2.10 Assume that $L=\{L(t): t \geq 0\}$ is an Z-valued Lévy process defined on a probability space $(\Omega, \mathcal{F}, \mathbb{P})$ with the system of generating triplets $\left\{\delta_{0}, t v, 0\right\}$. Let us define a measure $\tilde{v}$ on $(0, \infty) \times(Z \backslash\{0\})$ by

$$
\tilde{v}((0, t] \times B)=v_{t}(B)=t v(B), \quad B \in \mathcal{B}(Z \backslash\{0\}) .
$$

Let $\Omega_{0} \in \mathcal{F}$ be such that for every $\omega \in \Omega_{0}$, the function

$$
[0, \infty) \ni t \mapsto L(t, \omega) \in Z
$$

is càdlàg. Define a map $N$ by

$$
N(B, \omega):= \begin{cases}\#\{s:(s, L(s, \omega)-L(s-, \omega)) \in B\}, & \text { if } \omega \in \Omega_{0}, \\ 0 & \text { if } \omega \in \Omega \backslash \Omega_{0} .\end{cases}
$$

Then

- $\quad$ the function $N$ is a time-homogenous Poisson random measure on $(0, \infty) \times(Z \backslash\{0\})$ with intensity measure $\tilde{v}=$ Leb $\otimes v$,

- there exists a set $\Omega_{1} \in \mathcal{F}$ with $\mathbb{P}\left(\Omega_{1}\right)=1$ such that for every $\omega \in \Omega_{1}$ the following hold:

(1) for every $\varepsilon>0$ and $t \in(0, \infty)$ the measure $N(\cdot, \omega)$ restricted to $(0, t] \times\left(Z \backslash U_{\varepsilon}\right)$ is supported on a finite number of points, each of which has $N(\cdot, \omega)$-measure 1 ;

(2) for every $s \in(0, \infty), N(\{s\} \times(Z \backslash\{0\}), \omega)$ is equal to 0 or 1 ,

- $\quad$ there exists a set $\Omega_{3} \in \mathcal{F}$ with $\mathbb{P}\left(\Omega_{3}\right)=1$ such that for every $\omega \in \Omega_{3}$,

$$
S_{\varepsilon}(t, \omega):=\int_{(0, t] \times\left(U_{1} \backslash U_{\varepsilon}\right)}[x N(d(s, x), \omega)-x \tilde{v}(d(s, x))]
$$


converges, as $\varepsilon \searrow 0$, to an element of the Skorohod space $D([0, \infty), Z)$, locally uniformly, i.e., uniformly on any bounded interval $\subset[0, \infty)$,

- the $Z$-valued process $Y=\left\{Y_{t}: t \geq 0\right\}$ defined, for $\omega \in \Omega_{3}^{1}:=\Omega_{3} \cap \Omega_{1}$ by

$$
Y_{t}(\omega):=\lim _{\varepsilon \searrow 0} S_{\varepsilon}(t, \omega)+\int_{(0, t] \times\left(Z \backslash U_{1}\right)} x N(d(s, x), \omega)
$$

is a Lévy process with generating triplets $\{0, t v, 0\}$. In particular, the process $Y$ is identical in law with the process $L$.

Remark 2.11 We have seen that since $L$ is a Lévy process, $v_{t}=t v$. This implies as above that $N$ is a time-homogenous Poisson random measure. Moreover, the equality (2.20) can be written as,

$$
S_{\varepsilon}(t, \omega):=\int_{(0, t] \times\left(U_{1} \backslash U_{\varepsilon}\right)}[x N(d(s, x), \omega)-x d s v(d x)] .
$$

The above theorem enables us to define an Itô integral with respect to the Lévy process $L$ in terms of the corresponding compensated Poisson random measure $\tilde{N}$,

$$
\begin{aligned}
\int_{0}^{t} G(s) d L(s) & :=\int_{(0, t] \times U_{1}} G(s) x \tilde{N}(d(s, x)) \\
& +\int_{(0, t] \times\left(Z \backslash U_{1}\right)} G(s) x N(d(s, x)),
\end{aligned}
$$

where $G$ is an appropriate process taking values in the space $\mathcal{L}(Z, V)$, where $V$ is an appropriate Banach space.

From now we will consider only such processes that $N$ restricted to $(0, \infty) \times\left(Z \backslash U_{1}\right)$ is equal to 0 .

Given a process $G$ as above, we can define a process $\xi$

$$
\xi:[0, \infty) \times \Omega \ni(t, \omega) \mapsto\left\{Z \ni x \mapsto 1_{U_{1}}(x) G(s) x \in V\right\} \in L^{0}(Z, v, V)
$$

In view of Section 2.2, the integral $\int_{0}^{t} G(s) d L(s)$ is well defined for all $t \in[0, T]$, provided $V$ is a martingale type $p$ Banach space for some $p \in(1,2]$ and the process $\xi$ defined above takes values in $L^{0}(Z, v, V)$ and belongs to the class $\mathcal{M}^{p}\left(0, T, L^{p}(Z, v ; V)\right)$. Moreover, for every $q \in(1, p]$, there exists a constant $C>0$ independent of $G$ such that

$$
\begin{aligned}
\mathbb{E}\left|\sup _{t \in[0, T]} \int_{0}^{t} G(s) d L(s)\right|_{V}^{q} & \leq C \mathbb{E}\left(\int_{0}^{T} \int_{Z}|\xi(r, x)|_{V}^{p} v(d x) d r\right)^{q / p} \\
& =C \mathbb{E}\left(\int_{0}^{T} \int_{U_{1}}|G(r) x|_{V}^{p} v(d x) d r\right)^{q / p} .
\end{aligned}
$$

In fact, using the above approach, we can extend the definition of the Itô integral with respect to the Lévy process to the integrands belonging to the whole class $\mathcal{M}^{p}\left(0, T, L^{p}(Z, v ; V)\right)$ by

$$
\int_{0}^{t} \xi(s) d L(s):=\int_{(0, t] \times U_{1}} \xi(s) \tilde{N}(d(s, x)) .
$$

We finish this section with a result somehow converse to Theorem 2.10 but whose proof, in the finite-dimensional case, can be traced to the Proof of Theorem 19.2 in [70, pp.132134]. As we have observed earlier, this proof generalises to an infinite dimensional setting. 
Theorem 2.12 Assume that on a probability space $(\Omega, \mathcal{F}, \mathbb{P}), N$ is a time-homogenous Poisson random measure on $(0, \infty) \times(Z \backslash\{0\})$, where $Z$ is a separable Banach space, with intensity measure Leb $\otimes v$. Then

- there exists a set $\Omega_{1} \in \mathcal{F}$ with $\mathbb{P}\left(\Omega_{1}\right)=1$ such that for every $\omega \in \Omega_{1}$ the following hold:

(1) for every $\varepsilon>0$ and $t \in(0, \infty)$ the measure $N(\cdot, \omega)$ restricted to $(0, t] \times\left(Z \backslash U_{\varepsilon}\right)$ is supported on a finite number of points, each of which has $N(\cdot, \omega)$-measure 1 ;

(2) for every $s \in(0, \infty), N(\{s\} \times(Z \backslash\{0\}), \omega)$ is equal to 0 or 1 ,

- $\quad$ there exists a set $\Omega_{3} \in \mathcal{F}$ with $\mathbb{P}\left(\Omega_{3}\right)=1$ such that for every $\omega \in \Omega_{3}$, as $\varepsilon \searrow 0$,

$$
S_{\varepsilon}(t, \omega):=\int_{(0, t] \times\left(U_{1} \backslash U_{\varepsilon}\right)}[x N(d(s, x), \omega)-x d s v(d x)]
$$

converges to an element of the Skorohod space $D([0, \infty), Z)$, locally uniformly, i.e., uniformly on any bounded interval $\subset[0, \infty)$,

- $\quad$ an $Z$-valued process $Y=\{Y(t): t \geq 0\}$ defined, for $\omega \in \Omega_{3}^{1}:=\Omega_{1} \cap \Omega_{3}$ by

$$
Y(t, \omega):=\lim _{\varepsilon \searrow 0} S_{\varepsilon}(t, \omega)+\int_{(0, t] \times\left(Z \backslash U_{1}\right)} x N(d(s, x), \omega)
$$

is a Lévy process with generating triplets $\{0, t v, 0\}$.

The details of the results presented in this subsection will be dealt in a separate publication [16].

\section{Martingale Solutions of Stochastic Reaction-diffusion Equations}

\subsection{Statements of the Main Results}

In this section we will state our main results. For this purpose, we will introduce the problem, the concept of a martingale solution, and the main assumptions.

Let the Banach spaces $E, X$ and the linear operator $A$ be as in Assumption 1. We also assume that we have a probability space $(\Omega, \mathcal{F}, \mathbb{P})$ and Poisson random measure $\eta$ as in Assumption 2 and Definition 2.3. Throughout we fix $T>0$.

We consider the following stochastic evolution equation:

$$
\left\{\begin{array}{l}
d u(t)+A u(t) d t=F(t, u(t)) d t+\int_{Z} G(t, u(t) ; z) \tilde{\eta}(d z, d t), \quad t \in(0, T], \\
u(0)=u_{0} \in X .
\end{array}\right.
$$

where $\tilde{\eta}$ is the compensated Poisson random measure corresponding to $\eta$, see Definition 2.3.

Using the notation introduced in Notation 2, the assumptions on the nonlinear map $G$ read as follows.

Assumption 3 There exists $\rho \in\left(0, \frac{1}{p}\right)$ and a bounded and separately continuous map

$$
G:[0, T] \times X \rightarrow L^{p}\left(Z, v, D\left(A^{\rho-\frac{1}{p}}\right)\right) .
$$

Let us observe that this implies that the map $A^{\rho-\frac{1}{p}} G:[0, T] \times X \rightarrow L^{p}(Z, v, E)$ is well defined. In what follows, we will use the latter instead of $G$. 
Next we will present assumptions on the drift operator $F$. For this purpose we first recall the notion of the subdifferential of the norm $\varphi$, for more detail see [23].

Given $x, y \in X$ the map $\varphi: \mathbb{R} \ni s \mapsto|x+s y| \in \mathbb{R}$ is convex and therefore is right and left differentiable. Let us denote by $D_{ \pm}|x| y$ the right/left derivative of $\varphi$ at 0 . Then the subdifferential $\partial|x|$ of $|x|, x \in X$, is defined by

$$
\partial|x|:=\left\{x^{*} \in X^{*}: D_{-}|x| y \leq\left\langle y, x^{*}\right\rangle \leq D_{+}|x| y, \quad y \in X\right\},
$$

where $X^{*}$ is the dual space to $X$. One can show that not only $\partial|x|$ is a nonempty, closed and convex set, but also

$$
\partial|x|=\left\{x^{*} \in X^{*}:\left\langle x, x^{*}\right\rangle=|x| \text { and }\left|x^{*}\right| \leq 1\right\} .
$$

In particular, $\partial|0|$ is the unit ball in $X^{*}$.

Assumption 4 (i) The map $F:[0, T] \times X \rightarrow X$ is separately continuous.

(ii) There exist numbers $k_{0}>0, q>1$ and $k \geq 0$ such that with

$$
a(r)=k_{0}\left(1+r^{q}\right), r \geq 0,
$$

the following condition holds for $t \in[0, T]$,

$$
\langle-A x+F(t, x+y), z\rangle \leq a\left(|y|_{X}\right)-k|x|_{X}, \quad x \in D(A), y \in X, z \in \partial|x| .
$$

(iii) There exists a sequence $\left(F_{n}\right)_{n \in \mathbb{N}}$ of bounded separately continuous maps from $[0, T] \times X$ to $X$ such that

(a) $F_{n}$ satisfies condition (ii) above uniformly in $n$,

(b) $F_{n}$ converges in $X$ pointwise on $[0, T] \times X$ to $F$.

With all the notations and concepts presented above we are ready to define a martingale solution to the problem (3.1). Let us add a remark that is surely obvious to many readers while leaving it out could lead to a confusion for some other readers. Although in order to present the problem we have used a probability space and a PRM, these two objects are part of the solution. The only given objects are the space $(Z, \mathcal{Z})$ and measure $v \in M_{+}(Z)$.

Definition 3.1 Let us assume that $E$ and $X$ are Banach spaces satisfying parts 1(i), 2(i) and 2(ii) of Assumption 1. Let us also assume that $v$ is a $\sigma$-finite nonnegative measure on a measurable space $(Z, \mathcal{Z})$, i.e., $v \in M_{+}(Z)$. Let $p \in(1,2]$ a real number as in part 1(i) of Assumption 1 .

An $X$-valued martingale solution to the problem (3.1) is a system

$$
(\Omega, \mathcal{F}, \mathbb{P}, \mathbb{F}, \eta, u)
$$

such that

(i) $(\Omega, \mathcal{F}, \mathbb{F}, \mathbb{P})$ is a complete filtered probability space with a filtration $\mathbb{F}=\left\{\mathcal{F}_{t}: t \in\right.$ $[0, T]\}$ satisfying the usual conditions,

(ii) $\quad \eta$ is a time-homogeneous Poisson random measure on $(Z, \mathcal{B}(Z))$ with intensity measure $v \otimes \operatorname{Leb}$ over $(\Omega, \mathcal{F}, \mathbb{F}, \mathbb{P})$, 
(iii) $u:[0, T] \times \Omega \rightarrow X$ is an $\mathbb{F}$-progressively measurable process such that for any $t \in[0, T], \mathbb{P}$ a.s.

$$
\begin{array}{r}
\int_{0}^{t}\left|e^{-(t-r) A} F(r, u(r))\right|_{X} d r<\infty, \\
\int_{0}^{t} \int_{Z}\left|e^{-(t-r) A} G(r, u(r) ; z)\right|_{E}^{p} v(d z) d r<\infty,
\end{array}
$$

and for any $t \in[0, T], \mathbb{P}$ a.s.,

$$
\begin{aligned}
u(t)= & e^{-t A} u(0)+\int_{0}^{t} e^{-(t-r) A} F(r, u(r)) d r \\
& +\int_{0}^{t} \int_{Z} e^{-(t-r) A} G(r, u(r) ; z) \tilde{\eta}(d z, d r) .
\end{aligned}
$$

If in addition there exists a separable Banach space $\mathbf{B}$ such that

$$
u \in \mathbb{D}([0, T] ; \mathbf{B}), \quad \mathbb{P} \text {-a.s. }
$$

then the system (3.3) will be called an $X$-valued martingale solution to problem (3.1) with càdlàg paths in $\mathbf{B}$.

We will say that the $X$-valued martingale solution to problem (3.1) with càdlàg paths in $\mathbf{B}$ is unique iff for any other martingale solution to Eq. 3.1 with càdlàg paths in $\mathbf{B}$

$$
\left(\Omega^{\prime}, \mathcal{F}^{\prime}, \mathbb{P}^{\prime}, \mathbb{F}^{\prime}, \eta^{\prime}, u^{\prime}\right),
$$

the laws of the processes $u$ and $u^{\prime}$ on the space $\mathbb{D}([0, T] ; \mathbf{B})$ are equal.

We refer to a recent paper [15] where the uniqueness in law of processes defined by stochastic convolutions with respect to PRM's are discussed.

We we formulate our main theorem.

Theorem 3.2 Let $p \in(1,2], E, X$ and $A$ be as in Assumption 1. Let $v$ be a $\sigma$-finite nonnegative measure on a measurable space $(Z, \mathcal{Z})$, i.e., $v \in M_{+}(Z)$. Let the nonlinear maps $G$ and $F$ satisfy Assumption 3 and Assumption 4, respectively. Assume that $X \subset$ $D\left(A^{\rho-\frac{1}{p}}\right)$ and that

$$
\text { the embedding } X \hookrightarrow D\left(A^{\rho-\frac{1}{p}}\right) \text { is continuous. }
$$

Let $q$ be the number from Assumption 4(ii). Assume that $q<q_{\max }$, where

$$
q_{\max }=\left\{\begin{array}{l}
\frac{p}{1-\rho p} \text { if }- \text { Agenerates a contraction type semigroup on } D\left(A^{\rho-\frac{1}{p}}\right), \\
\frac{p}{1-\rho} \text { otherwise. }
\end{array}\right.
$$

Assume also that there exists a separable UMD Banach space $Y$ such that

(1) $X \subset Y$,

(2) A has an extension $A_{Y}$ which satisfies the parts 1(iii)-1(v) of Assumption 1 on $Y$,

(3) and $D\left(A_{Y}^{\theta}\right) \subset X$ for some $\theta \leq 1-\frac{q}{q_{\max }}$.

Then, for any $u_{0} \in X$ problem (3.1) has an $X$-valued martingale solution with càdlàg paths in $D\left(A^{\rho-\frac{1}{p}}\right)$, see Definition 3.1. 
Moreover, for any $\tilde{q} \in\left(q, q_{\max }\right)$ and $r \in(1, p)$ the stochastic process $u$ satisfies

$$
\mathbb{E}\left(\int_{0}^{T}|u(t)|_{X}^{\tilde{q}} d t\right)^{\frac{r}{\tilde{q}^{2}}}<\infty .
$$

Remark 3.3 If the maps $F(t, \cdot)$ and $A^{\rho-\frac{1}{p}} G(t, \cdot)$ are Lipschitz continuous uniformly with respect to $t \in[0, T]$, i.e., there exists $K>0$ such that for all $t \in[0, T]$ and all $u_{1}, u_{2} \in X$,

$$
\begin{aligned}
\left|F\left(t, u_{2}\right)-F\left(t, u_{1}\right)\right|_{X} & \leq K\left|u_{2}-u_{1}\right|_{X}, \\
\int_{Z}\left|A^{\rho-\frac{1}{p}} G\left(t, u_{2} ; z\right)-A^{\rho-\frac{1}{p}} G\left(t, u_{1} ; z\right)\right|_{E}^{p} v(d z) & \leq K\left|u_{2}-u_{1}\right|_{X}^{p},
\end{aligned}
$$

then the SPDEs (3.1) has a unique strong solution. In our work we are interested in the case when both these conditions are relaxed.

In order to prove Theorem 3.2 we will consider an auxiliary problem for which we will prove an auxiliary existence result (see Theorem 3.4) which holds under more restrictive conditions than the ones stated above. More precisely, we require that the nonlinear maps $F$ and $G$ satisfy the following set of conditions.

Assumption 5 There exists $\rho \in\left[0, \frac{1}{p}\right.$ ) such that the (nonlinear) maps

$$
\begin{aligned}
& F:[0, T] \times X \rightarrow D\left(A^{\rho-1}\right), \\
& G:[0, T] \times X \rightarrow L^{p}\left(Z, \nu, D\left(A^{\rho-\frac{1}{p}}\right)\right),
\end{aligned}
$$

are bounded and separately continuous.

Remark The above assumption can also be stated in a more precise way, see Assumption 3 . To be precise, we could request that there exists $\rho \in\left[0, \frac{1}{p}\right)$ such that the maps

$$
\begin{aligned}
& A^{\rho-1} F:[0, T] \times X \rightarrow E, \\
& A^{\rho-\frac{1}{p}} G:[0, T] \times X \rightarrow L^{p}(Z, v, E),
\end{aligned}
$$

are bounded and separately continuous.

We state the following theorem whose proof will be given in Section 8. Although it is only an auxiliary result, it is still important as it is the main tool for the proof of Theorem 3.2 and we are not aware of a related result in the existing literature.

Theorem 3.4 Let $E$ and $A$ be as in Assumption 1 and $(Z, \mathcal{Z}, v)$ be a measure space with $v \in M_{+}(Z)$. Let the hypothesis in Assumption 5 be satisfied. Then, for every $u_{0} \in D\left(A^{\rho-\frac{1}{p}}\right)$ problem (3.1) has an E-valued martingale solution

$$
(\Omega, \mathcal{F}, \mathbb{P}, \mathbb{F}, \eta, u)
$$

with càdlàg trajectories in $D\left(A^{\rho-\frac{1}{p}}\right)$.

Moreover, the stochastic process $u$ satisfies

$$
\int_{0}^{T} \mathbb{E}|u(t)|_{E}^{p} d t<\infty .
$$

In view of Section 2.3, Theorem 3.4 can be written in terms of a Lévy process as follows. 


\subsection{Formulation of our Results in Terms of Lévy Processes}

Let $Z$ be a separable Banach space with the Borel $\sigma$-field $\mathcal{Z}=\mathcal{B}(Z)$. Assume that $L=$ $\{L(t): t \geq 0\}$ is a $Z$-valued Lévy process with generating triple $\left(\delta_{0}, t v, 0\right)$, see Theorem 2.10 , such that for some fixed $p \in(1,2]$,

$$
\int_{U_{1}}|z|_{Z}^{p} v(d z)<\infty
$$

where as in Section 2.3, $U_{1}$ is the closed unit ball in $Z$. Note that if supp $v \subset U_{1}$, then the Poisson random measure $N$ corresponding to $L$ restricted to $(0, \infty) \times\left(Z \backslash U_{1}\right)$ is equal to 0 .

Here, instead of Assumption 3 we assume the following set of hypotheses.

Assumption 6 There exists $\rho \in\left(0, \frac{1}{p}\right)$ such that the diffusion coefficient $\mathbb{G}$ is bounded and separately continuous map

$$
A^{\rho-\frac{1}{p}} \mathbb{G}:[0, T] \times X \rightarrow \mathcal{L}(Z, E) .
$$

Remark Let us notice that this framework is less general than the one of Poisson random measures. In particular, if the map $\mathbb{G}$ satisfies Assumption 6 then the map $G$ defined by

$$
G:[0, T] \times E \ni(t, u) \mapsto\left\{Z \ni z \mapsto 1_{U_{1}}(z) \mathbb{G}(t, u) z\right\} \in L^{p}(Z, v ; E) .
$$

satisfies, in view of Eq. 3.13 and the continuous embedding $E \subset X$, Assumption 3 .

We consider the following stochastic evolution equation

$$
\left\{\begin{array}{l}
d u(t)+A u(t) d t=F(t, u(t)) d t+\mathbb{G}(t, u(t)) d L(t), \\
u(0)=u_{0} .
\end{array}\right.
$$

In view of above remark and Section 2.3 we get the following result which is crucial in our reformulation of Theorem 3.4 in terms of Lévy processes.

Theorem 3.5 Assume that the Banach space E, the linear map A and the map $F$ satisfy the assumptions of Theorem 3.4. Let us assume that $Z$ is a separable Banach space and $Y=$ $\{Y(t): t \geq 0\}$ is an $Z$-valued Lévy process defined on a probability space $\left(\Omega^{0}, \mathcal{F}^{0}, \mathbb{P}^{0}\right)$ with the system of generating triplets $\left\{\delta_{0}, t v, 0\right\}$ such that supp $v \subset U_{1}$ and, for some $p \in(1,2]$, the condition (3.13) is satisfied. Assume that the map $\mathbb{G}$ satisfies Assumption 6. Then, for every $u_{0} \in D\left(A^{\rho-\frac{1}{p}}\right)$ there exists a system

$$
(\Omega, \mathcal{F}, \mathbb{P}, \mathbb{F}, L, u)
$$

such that

(i) $(\Omega, \mathcal{F}, \mathbb{F}, \mathbb{P})$ is a complete filtered probability space with filtration $\mathbb{F}=\left\{\mathcal{F}_{t}\right\}_{t \in[0, T]}$ satisfying the usual conditions;

(ii) $L=\{L(t): t \in[0, T]\}$ is a $Z$-valued Lévy process with Lévy measure $v$ over $(\Omega, \mathcal{F}, \mathbb{F}, \mathbb{P})$;

(iii) $u=\{u(t): t \in[0, T]\}$ is an E-valued and adapted process, with $D\left(A^{\rho-\frac{1}{p}}\right)$-valued càdlàg paths, such that

$$
\int_{0}^{T} \mathbb{E}|u(t)|_{E}^{p} d t<\infty
$$


$(F(t, u(t)): t \in[0, T])$ and $(\mathbb{G}(t, u(t)): t \in[0, T])$ are well defined $D\left(A^{\rho-\frac{1}{p}}\right)$ valued, resp. $L^{p}\left(Z, v ; D\left(A^{\rho-\frac{1}{p}}\right)\right)$-valued and progressively measurable processes, and for all $t \in[0, T], \mathbb{P}$-a.s.

$$
\begin{array}{r}
u(t)=e^{-t A} u_{0}+\int_{0}^{t} e^{-(t-r) A} F(r, u(r)) d r \\
+\int_{0}^{t} e^{-(t-r) A} \mathbb{G}(r, u(r)) d L(r) .
\end{array}
$$

Proof of Theorem 3.5 This result readily follows from Theorem 3.4 because of the following argument.

Let us consider a separable Banach space $Z$ and an $Z$-valued Lévy process $Y=\{Y(t)$ : $t \geq 0\}$, defined on a probability space $\left(\Omega^{0}, \mathcal{F}^{0}, \mathbb{P}^{0}\right)$ with the system of generating triplets $\left\{\delta_{0}, t v, 0\right\}$ such that $\operatorname{supp} v \subset U_{1}$, and condition (3.13) is satisfied for some $p \in(1,2]$. Let $N$ be the corrsponding Poisson random measure given by Theorem 2.10.

Let us fix $u_{0} \in D\left(A^{\rho-\frac{1}{p}}\right)$. Since the map $\mathbb{G}$ satisfies Assumption 6, by Theorem 3.4, there exists a system

$$
(\Omega, \mathcal{F}, \mathbb{P}, \mathbb{F}, \eta, u)
$$

which is an $E$-valued martingale solution problem (3.1) (with càdlàg trajectories in $D\left(A^{\rho-\frac{1}{p}}\right)$ ). In particular, $\eta$ is a time-homogeneous Poisson random measure on a Banach space $Z$ with the intensity measure $v \otimes$ Leb such that condition (3.13) is satisfied. Applying Theorem 2.12 we can find a $Z$-valued Lévy process $L=\{L(t): t \geq 0\}$ with the generating triplets $\left\{\delta_{0}, t v, 0\right\}$. By the results discussed in Section 2.3 we infer that the system

$$
(\Omega, \mathcal{F}, \mathbb{P}, \mathbb{F}, \eta, L)
$$

is a martingale solution to problem (3.15).

\subsection{Outline of the Proof of Theorems 3.2 and 3.4}

The detail of the proofs of the theorems 3.2 and 3.4 are given in Sections 9 and 8, respectively. These proofs are very technical and to make the reading of the paper easy, we outline the proofs of Theorem 3.4 and Theorem 3.2 in this subsection.

Outline of the proof of Theorem 3.4 The proof relies on a combination of approximation and compactness methods. Namely, we approximate the initial condition $u_{0}$ by a sequence $\left(x_{n}\right)_{n \in \mathbb{N}} \subset E$ satisfying

$$
x_{n} \rightarrow u_{0} \text { strongly in } D\left(A^{\rho-\frac{1}{p}}\right),
$$

as $n \rightarrow \infty$. We also define a sequence $\left(u_{n}\right)_{n \in \mathbb{N}}$ of adapted $E$-valued processes by

$$
\begin{aligned}
u_{n}(t)= & e^{-t A} x_{n}+\int_{0}^{t} e^{-(t-s) A} F\left(s, \hat{u}_{n}(s)\right) d s \\
& +\int_{0}^{t} \int_{Z} e^{-(t-s) A} G\left(s, \hat{u}_{n}(s) ; z\right) \tilde{\eta}(d z ; d s), \quad t \in[0, T],
\end{aligned}
$$

where $\hat{u}_{n}$ is defined by

$$
\hat{u}_{n}(s):= \begin{cases}x_{n}, & \text { if } s \in\left[0,2^{-n}\right), \\ f_{\phi_{n}(s)-2^{-n}}^{\phi_{n}(s)} u_{n}(r) d r, & \text { if } s \geq 2^{-n},\end{cases}
$$


and $\phi_{n}:[0, \infty) \rightarrow[0, \infty)$ is a function defined by $\phi_{n}(s)=\frac{k}{2^{n}}$, if $k \in \mathbb{N}$ and $\frac{k}{2^{n}} \leq s<\frac{k+1}{2^{n}}$, i.e., $\phi_{n}(s)=2^{-n}\left[2^{n} s\right], s \geq 0$, where $[t]$ is the integer part of $t \in \mathbb{R}$. Here we have used the following shortcut notation

$$
f_{A} f(t) d t:=\frac{1}{\operatorname{Leb}(A)} \int_{A} f(t) d t, \quad A \in \mathcal{B}([0, T]),
$$

where Leb denotes the Lebesgue measure. Let us point out that between the grid points, Eq. 3.18 is linear, therefore, $u_{n}$ is well defined for all $n \in \mathbb{N}$.

Secondly, we proved that for any $\alpha \in(0, \rho)$ and $\rho^{\prime} \in(0, \rho)$ there exists a constant $C$ such that the following inequalities hold

$$
\begin{aligned}
\sup _{n \in \mathbb{N}} \mathbb{E}\left\|A^{\rho^{\prime}} u_{n}\right\|_{L^{p}(0, T ; E)}^{p} \leq C, \\
\sup _{n \in \mathbb{N}} \mathbb{E}\left\|A^{\rho^{\prime}} \hat{u}_{n}\right\|_{L^{p}(0, T ; E)}^{p} \leq C, \\
\sup _{n \in \mathbb{N}} \mathbb{E}\left\|u_{n}\right\|_{W^{\alpha, p}(0, T ; E)}^{p} \leq C .
\end{aligned}
$$

The proofs of these uniform estimates are non-trivial and rely on Lemmata 7.2 and 7.4, Proposition E.1 and the maximal regularity for deterministic parabolic equations.

Thirdly, by defining a sequence of Poisson random measures $\left(\eta_{n}\right)_{n \in \mathbb{N}}$ by putting $\eta_{n}=\eta$ for all $n \in \mathbb{N}$, we will prove that for any $\rho^{\prime} \in(0, \rho)$ the family of the laws of random variables $\left(\left(u_{n}, \eta_{n}\right)\right)_{n \in \mathbb{N}}$ is tight on the cartesian product space $\left[L^{p}(0, T ; E) \cap\right.$ $\left.\mathbb{D}\left([0, T] ; D\left(A^{\rho^{\prime}-1}\right)\right)\right] \times M_{I}(Z \times[0, T])$. Because a cartesian product of two compact sets is compact, it is sufficient to consider the tightness of the components of the sequence $\left(\left(u_{n}, \eta_{n}\right)\right)_{n \in \mathbb{N}}$.

For this aim let us define two auxiliary sequences of stochastic processes by

$$
\begin{aligned}
& f_{n}(t)=F\left(t, \hat{u}_{n}(t)\right), \quad t \in[0, T], \\
& v_{n}(t)=\int_{0}^{t} \int_{Z} e^{-(t-s) A} G\left(s, \hat{u}_{n}(s) ; z\right) \tilde{\eta}(d z ; d s), \quad t \in[0, T] .
\end{aligned}
$$

The tightness of laws of the processes $\left(u_{n}\right)_{n \in \mathbb{N}}$ on $L^{p}(0, T ; E) \cap \mathbb{D}\left([0, T] ; D\left(A^{\rho^{\prime}-1}\right)\right)$ follows by observing that

$$
u_{n}=v_{n}+\Lambda^{-1} f_{n}+e^{-\cdot A} x_{n}, \quad n \in \mathbb{N},
$$

and using Lemma 2.2 (for $\Lambda^{-1} f_{n}$ ) and the Lemmata 7.6 and 7.7 (for $v_{n}$ ). The tightness of the laws family of $\left(\eta_{n}\right)_{n \in \mathbb{N}}$ follows from [62, Theorem 3.2].

This tightness result along with the Prokhorov theorem and the modified Skorohod Representation Theorem, see Theorem C.1, implies that there exist a probability space $(\hat{\Omega}, \hat{\mathcal{F}}, \hat{\mathbb{P}})$ and $L^{p}(0, T ; E) \cap \mathbb{D}\left([0, T] ; D\left(A^{\rho^{\prime}-1}\right)\right) \times M_{I}(Z \times[0, T])$ - valued random variables $\left(u_{*}, \eta_{*}\right),\left(\hat{u}_{n}, \hat{\eta}_{n}\right), n \in \mathbb{N}$, such that $\hat{\mathbb{P}}$-a.s.

$$
\left(\hat{u}_{n}, \hat{\eta}_{n}\right) \rightarrow\left(u_{*}, \eta_{*}\right)
$$

in $L^{p}(0, T, E) \cap \mathbb{D}\left([0, T], D\left(A^{\rho^{\prime}-1}\right)\right) \times M_{I}(Z \times[0, T]), \hat{\eta}_{n}=\eta_{*}$, and

$$
\mathcal{L}\left(\left(\hat{u}_{n}, \hat{\eta}_{n}\right)\right)=\mathcal{L}\left(\left(u_{n}, \eta_{n}\right)\right),
$$

for all $n \in \mathbb{N}$. Taking the new filtration $\hat{\mathbb{F}}$ as the natural filtration of $\left(\hat{u}_{n}, \hat{\eta}_{n}, u_{*}, \eta_{*}\right)$ we prove that over the filtered probability space $(\hat{\Omega}, \hat{\mathcal{F}}, \hat{\mathbb{F}}, \hat{\mathbb{P}})$ the objects $\hat{\eta}_{n}$ and $\eta_{*}$ are 
time-homogeneous Poisson random measure with intensity measure $v \otimes$ Leb. We also prove that in appropriate topology

$$
\hat{u}_{n}-e^{-\cdot A} x_{n}-\Lambda^{-1} F_{n}\left(\cdot, \hat{u}_{n}(\cdot)\right) d s-\hat{v}_{n}=0,
$$

where a process $\hat{v}_{n}$ is defined analogously to the way we have defined the process $v_{n}$ by replacing $u_{n}$ with $\hat{u}_{n}$. Using (3.20) and the uniform a priori estimates we obtain earlier we can pass to the limit and derive that $\hat{\mathbb{P}}$-a.s.

$$
u_{*}(\cdot)=e^{-\cdot A} u_{0}+\int_{0}^{\cdot} e^{-(t-s) A} F\left(s, u_{*}(s)\right) d s+\int_{0}^{\cdot} \int_{Z} e^{-(t-s) A} G\left(s, u_{*}(s)\right) \tilde{\eta}_{*}(d z, d s) .
$$

This ends the proof of Theorem 3.4.

The scheme of the proof of Theorem 3.2 is very similar to the above idea, but it is longer and more complicated. In the next paragraph we will simply outline the main ideas of the proof and refer the reader to Section 9 for more details.

Outline of the proof of Theorem 3.2 The proof of Theorem 3.2 also relies on approximation and compactness methods. We mainly exploit the Assumption 4(iii) to set in the Banach space $E$ an approximating problem with bounded coefficients. This approximating (auxiliary) problem takes the form

$$
\left\{\begin{array}{l}
d u_{n}(t)+\left(A u_{n}(t)+F_{n}\left(t, u_{n}(t)\right) d t=\int_{Z} G\left(t, u_{n}(t)\right) \tilde{\eta}(d z, d t),\right. \\
u_{n}(0)=u_{0},
\end{array}\right.
$$

which, thanks to Theorem 3.4, has an $E$-valued martingale solution with càdlàg paths in $D\left(A^{\rho-\frac{1}{p}}\right)$. We denote this martingale solution by

$$
\left(\Omega_{n}, \mathcal{F}_{n}, \mathbb{F}_{n}, \mathbb{P}_{n}, \eta_{n}, u_{n}\right)
$$

The stochastic process $u_{n}$ can be written in the form

$$
\begin{aligned}
u_{n}(t)=e^{-t A} u_{0}+ & \int_{0}^{t} e^{-(t-s) A} F_{n}\left(s, u_{n}(s)\right) d s \\
& +\int_{0}^{t} \int_{Z} e^{-(t-s) A} G\left(s, u_{n}(s)\right) \tilde{\eta}_{n}(d z, d s) \\
= & e^{-t A} u_{0}+z_{n}(t)+v_{n}(t) .
\end{aligned}
$$

The first step of the proof is to derive uniform a priori estimates concerning the convolution processes $z_{n}$ and $v_{n}$. In this step the results obtained in Section 7, especially Lemmata 7.9 and 10, will play an important role. In fact, thanks to parts 1(i)-1(v) of Assumption 1, we can apply these results and deduce that for any $\tilde{q} \in\left(q, q_{\max }\right)$ and $r \in(1, p)$,

$$
\sup _{n \in \mathbb{N}} \mathbb{E}_{n}\left[\left\|v_{n}\right\|_{L^{\tilde{q}}(0, T ; E)}^{r}+\sup _{t \in[0, T]}\left|z_{n}(t)\right|_{X}^{\frac{r}{\tilde{q}}}\right]<\infty .
$$

Let us fix $\tilde{q} \in\left(q, q_{\max }\right)$ and $\rho^{\prime} \in(0, \rho)$. We set $\mathbf{B}_{0}=D\left(A^{\rho^{\prime}-1}\right)$ and put

$$
\mathfrak{X}_{T}=C([0, T] ; X) \times L^{\tilde{q}}(0, T ; E) \cap \mathbb{D}\left([0, T] ; \mathbf{B}_{0}\right) \times M_{I}(Z \times[0, T]) .
$$

In the following step we will prove that the family of laws of $\left(z_{n}, v_{n}, \eta_{n}\right)_{n \in \mathbb{N}}$ is tight on $\mathfrak{X}_{T}$. For this aim we will prove that the laws of the sequence $\left(v_{n}\right)_{n \in \mathbb{N}}$, respectively $\left(\eta_{n}\right)_{n \in \mathbb{N}}$, are tight on $L^{\tilde{q}}(0, T ; E) \cap \mathbb{D}\left([0, T] ; \mathbf{B}_{0}\right)$, respectively $\left.M_{I}(Z \times[0, T])\right)$. The tightness of laws of the sequence $\left(v_{n}\right)_{n \in \mathbb{N}}$ is a consequence of Lemmata 7.6 and 7.7. The tightness of the family of laws of $\left(\eta_{n}\right)_{n \in \mathbb{N}}$ is a consequence of [62, Theorem 3.2]. The tightness of family of laws of the sequence $\left(z_{n}\right)_{n \in \mathbb{N}}$ on $C([0, T] ; X)$ is difficult to prove. A proof of this fact 
relies very much on the hypotheses (3.2)-(3.2) in Theorem 3.2. Firstly we will need to use Lemma 9.1, see [23] for a proof, as well as the previous uniform estimates, to prove that for some $\tilde{p} \in\left(1, \frac{q_{\max }}{q}\right) \sup _{n \in \mathbb{N}}\left|F_{n}\left(\cdot, u_{n}(\cdot)\right)\right|_{L \tilde{p}(0, T ; Y)}$ is bounded in probability. Observing that $z_{n}=\Lambda^{-1} F_{n}\left(\cdot, u_{n}(\cdot)\right)$ and using Lemma 2.2, which is applicable thanks to parts $1(\mathrm{i})-1(\mathrm{v})$ of Assumption 1, we will deduce that for some $\theta \leq 1-\frac{1}{\tilde{p}}$ the family of laws of $\left(z_{n}\right)_{n \in \mathbb{N}}$ is tight on $C\left([0, T] ; D\left(A_{Y}^{\theta}\right)\right)$ and hence on $C([0, T] ; X)$.

This along with the Prokhorov Theorem and the modified Skorohod Representation Theorem, see Theorem C.1, imply that there exist a probability space $(\hat{\Omega}, \hat{\mathcal{F}}, \hat{\mathbb{P}})$ and $\mathfrak{X}_{T}$ - valued random variables $\left(z_{*}, v_{*}, \eta_{*}\right),\left(\hat{z}_{n}, \hat{v}_{n}, \hat{\eta}_{n}\right), n \in \mathbb{N}$, such that $\hat{\mathbb{P}}_{\text {-a.s. }}$

$$
\left(\hat{z}_{n}, \hat{v}_{n}, \hat{\eta}_{n}\right) \rightarrow\left(z_{*}, v_{*}, \eta_{*}\right) \text { in } \mathfrak{X}_{T},
$$

and, for all $n \in \mathbb{N}, \hat{\eta}_{n}=\eta_{*}$ and

$$
\mathcal{L}\left(\left(\hat{z}_{n}, \hat{v}_{n}, \hat{\eta}_{n}\right)\right)=\mathcal{L}\left(\left(z_{n}, v_{n}, \eta_{n}\right)\right) .
$$

Next we will carefully construct a new filtration $\hat{\mathbb{F}}$ and prove that over the new filtered probability space $(\hat{\Omega}, \hat{\mathcal{F}}, \hat{\mathbb{F}}, \hat{\mathbb{P}})$ the objects $\hat{\eta}_{n}$ and $\eta_{*}$ are time-homogeneous Poisson random measure with intensity measure $v \otimes$ Leb. We also prove that in appropriate topology

$$
\hat{u}_{n}-e^{-\cdot A} u_{0}-\int_{0}^{\cdot} e^{-(\cdot-s) A} F_{n}\left(s, \hat{u}_{n}(s)\right) d s-\int_{0}^{\cdot} e^{-(\cdot-s) A} G\left(s, \hat{u}_{n}(s)\right) \tilde{\hat{\eta}}(d z, d s)=0,
$$

where $\hat{u}_{n}=e^{-\cdot A} u_{0}+\hat{z}_{n}+\hat{v}_{n}$. Putting $u_{*}:=e^{-\cdot A} u_{0}+z_{*}+v_{*}$ and using (3.23) and the uniform a priori estimates we obtained earlier we can take the limit and deduce that $\hat{\mathbb{P}}$-a.s.

$$
u_{*}(\cdot)=e^{-\cdot A} u_{0}+\int_{0}^{\cdot} e^{-(t-s) A} F\left(s, u_{*}(s)\right) d s+\int_{0}^{\cdot} \int_{Z} e^{-(t-s) A} G\left(s, u_{*}(s)\right) \tilde{\eta}_{*}(d z, d s) .
$$

This ends the hardest part of the proof of Theorem 3.2. The scheme of the proof of Theorem 3.4 is very similar to the above idea and simpler. We refer the reader to Sections 9 and 8 for the omitted details.

\section{Application I: Reaction-diffusion Equations with Lévy Noise of the Spectral Type}

Throughout this section, we assume that $\mathcal{O}$ is a bounded open domain in $\mathbb{R}^{d}, d \in \mathbb{N}^{*}$, with $C^{\infty}$ boundary. We also fix real numbers $T>0, \alpha \in(0,2), p \in(1,2]$ and $q \in(1, \infty)$. Finally we assume that $L=\{L(t): t \in[0, \infty)\}$ is a real-valued tempered $\alpha$-stable Lévy process, i.e., a Lévy process with the Lévy measure $v_{\alpha}$ given by

$$
v_{\alpha}(d z)=1_{|z|<1}|z|^{-\alpha-1} e^{-|z|} d z, \quad z \in \mathbb{R} .
$$

Our aim in this section is to study an equation of the following type,

$$
\left\{\begin{aligned}
d u(t, \xi) & =\Delta u(t, \xi) d t-|u(t, \xi)|^{q-1} \operatorname{sgn}(u(t, \xi))+u(t, \xi) d t \\
& +\sqrt{|u(t, \xi)|} /(1+\sqrt{|u(t, \xi)|}) d L(t), \quad t \in(0, T], \xi \in \mathcal{O}, \\
u(t, \xi) & =0, \quad \xi \in \partial \mathcal{O}, t \in(0, T] \\
u(0, \xi) & =u_{0}(\xi), \quad \xi \in \mathcal{O},
\end{aligned}\right.
$$


We will achieve our aim by finding the conditions on the coefficients so that Theorem 3.2 is applicable. For this purpose we will reformulate problem (4.2) using a more general setting and the language of the Poisson random measures.

Firstly, we denote by $X=C_{0}(\overline{\mathcal{O}})$ the space of real continuous functions on $\overline{\mathcal{O}}$ which vanish on the boundary $\partial \mathcal{O}$. For $\gamma \in \mathbb{R}$ and $r \in(1, \infty)$ by the symbol $H^{\gamma, r}(\mathcal{O})$ we will denote the fractional order Sobolev space defined by mean of the complex interpolation method, see [73, Definition 1, page 301]. By $H_{0}^{\gamma, r}(\mathcal{O})$ we will denote the closure of the space $C_{0}(\mathcal{O})$ in $H^{\gamma, r}(\mathcal{O})$, see also [73, Definition 2, page 301]. We will simply write $H^{\gamma, r}$ and $H_{0}^{\gamma, r}$ when there is no risk of ambiguity.

Let us briefly recall the definitions of these spaces. If $k$ is a natural number and $p \in$ $[1, \infty)$ is a real number, we denote by $H^{k, p}(\mathcal{O})$, see [38, section I.6], the space of all functions $u \in L^{p}(\mathcal{O})$ whose weak derivatives $D^{\alpha} u$ of degree $|\alpha| \leq k$ exist and belong to $L^{p}(\mathcal{O})$. Endowed with a natural norm $\|\cdot\|_{k, p}$

$$
\|u\|_{k, p}^{p}:=\sum_{|\gamma| \leq k}\left|D^{\gamma} u\right|_{L^{p}}^{p}, \quad u \in H^{k, p}(\mathcal{O}),
$$

this space is a separable Banach space. The closure of the space $C_{0}^{\infty}(\mathcal{O})$ in the space $H^{k, p}(\mathcal{O})$ is denoted by $H_{0}^{k, p}(\mathcal{O})$. In the case $\beta \in \mathbb{R}^{+} \backslash \mathbb{N}$, the fractional order Sobolev spaces $H^{\beta, p}(\mathcal{O})$ can be defined by the complex interpolation method, i.e.,

$$
H^{\beta, p}(\mathcal{O})=\left[H^{k, p}(\mathcal{O}), H^{m, p}(\mathcal{O})\right]_{\vartheta},
$$

where $k, m \in \mathbb{N}, k<m$, and $\vartheta \in(0,1)$ satisfy $\beta=(1-\vartheta) k+\vartheta m$. It is well known, see e.g. [52, Theorem 11.1, chapter I] and [73, Theorem 1.4.3.2 on p. 317] that $H_{0}^{s, p}(\mathcal{O})=H^{s, p}(\mathcal{O})$ iff $s \leq \frac{1}{p}$.

We denote by $A$ the operator $-\Delta$ in $L^{p}(\mathcal{O})$ with the Dirichlet boundary conditions, i.e.,

$$
\left\{\begin{array}{l}
D(A)=H_{0}^{1, p}(\mathcal{O}) \cap H^{2, p}(\mathcal{O}), \\
A u=-\Delta u, \quad u \in D(A) .
\end{array}\right.
$$

Next, let us consider a separately continuous real valued functions $f$ defined on $[0, T] \times$ $\mathcal{O} \times \mathbb{R}$ satisfying the following condition. There exists a number $K>0$ such that for $t \geq 0, x \in \mathcal{O}, u \in \mathbb{R}$,

$$
-K\left(1+|u|^{q} 1_{[0, \infty)}(u)\right) \leq f(t, x, u) \leq K\left(1+|u|^{q} 1_{(-\infty, 0]}(u)\right) .
$$

It is not difficult to prove that if $f$ satisfies (4.5), then

$$
f(t, v+z) \operatorname{sgn}(v) \leq K\left(1+|z|^{q}\right), \text { for all } v, z \in \mathbb{R}, t \in[0, T] .
$$

Therefore, by [10, Proposition 6.2] the Nemytskii map $F$ defined by

$$
F(t, u)(\xi):=f(t, \xi, u(\xi)), u \in X, \xi \in \mathcal{O}, t \in[0, T],
$$

satisfies items (i) and (ii) of Assumption 4 on $X$.

Approximating $f$ by a sequence $\left(f_{n}\right)_{n=1}^{\infty}$, where for any $t \in[0, T]$ and $\xi \in \mathcal{O}$

$$
f_{n}(t, \xi, u):= \begin{cases}f(t, \xi, u), & \text { if } u \in[-n, n] \\ f(t, \xi, n), & \text { if } u \geq n, \\ f(t, \xi,-n), & \text { if } u \leq-n,\end{cases}
$$

we obtain a sequence $\left(F_{n}\right)_{n \in \mathbb{N}}$ defined by

$$
F_{n}:[0, T] \times X \ni(t, u) \mapsto\left\{\mathcal{O} \ni x \mapsto f_{n}(t, \xi, u(\xi))\right\} \in X,
$$

which satisfies Assumption 4(iii). 
Next we reformulate the noise appearing in the problem that we want to study. In view of the results of Section 2.3, see [1], to the real-valued Lévy process $L$ there corresponds a time-homogeneous Poisson random measure $\eta$ with Lévy measure $v_{\alpha}$ on $Z=\mathbb{R}$ defined by Eq. 4.1. Moreover, the Lévy processes $L=\{L(t): t \geq 0\}$ and $\tilde{L}=\{\tilde{L}(t): t \geq 0\}$, where

$$
\tilde{L}(t):=\int_{0}^{t} \int_{\mathbb{R}} z \tilde{\eta}(d z, d s), \quad t \geq 0,
$$

have the same law on $\mathbb{D}([0, \infty) ; \mathbb{R})$.

Now, let $g$ be a bounded and separately continuous function defined on $[0, T] \times \mathcal{O} \times \mathbb{R}$ and taking values in $\mathbb{R}$. Furthermore, we assume that $g(t, x, \cdot)$ is continuous uniformly w.r.t. $(t, x)$. Let $G$ be defined by

$$
G(t, u ; z)(\xi)=g(t, \xi, u(\xi)) z, \quad t \in[0, T], u \in L^{1}(\mathcal{O}), z \in \mathbb{R}, \xi \in \mathcal{O} .
$$

With this notation problem (4.2) can be rewritten in the following form

$$
\left\{\begin{array}{l}
d u(t)+A u(t) d t=F(u(t)) d t+\int_{\mathbb{R}} G(t, u, z) \tilde{\eta}(d z, d t), \quad t \in(0, T], \\
u(t)=u_{0} .
\end{array}\right.
$$

The following theorem is a corollary of Theorem 3.2.

Theorem 4.1 Assume that $\alpha \in(0,2), p \in(1,2], p>\alpha, d \in \mathbb{N}^{*}$. Let $v_{\alpha}$ be a Lévy measure on $\mathbb{R}$ given by Eq. 4.1. Assume that $q>1$ and let $r$ be a number satisfying

$$
r>\max \{q d, 2 d\} .
$$

Then, for any $u_{0} \in C_{0}(\mathcal{O})$ there exists a $C_{0}(\mathcal{O})$-valued martingale solution $(\Omega, \mathcal{F}, \mathbb{F}, \mathbb{P}, \eta, u)$ to problem (4.8) with càdlàg trajectories in $L^{r}(\mathcal{O})$ such that $\eta$ is a time-homogeneous Poisson random measure on $(\mathbb{R}, \mathcal{B}(\mathbb{R}))$ with intensity measure $v_{\alpha} \otimes$ Leb.

Proof Let us fix parameters $\alpha, p, d, q$ as in the assumptions. Next let us choose real numbers $r>\max \{q d, 2 d\}$ and $\kappa \in\left(\frac{d}{r}, \frac{1}{q}\right)$. Let us also choose $X=C_{0}(\mathcal{O}), E=H_{0}^{\kappa, r}$ and $\mathbf{B}=L^{r}$. Let us put $\delta=\frac{\kappa}{2}$. Then, since $\frac{1}{2} \leq \frac{1}{p}$ we infer that $\delta<\frac{1}{2 q}<\frac{1}{2}$ and $\delta<\frac{1}{p}$. We also deduce that

$$
q_{\max }=\frac{1}{\delta}>q .
$$

Next, we denote by $A=A_{r}$ the minus Laplace operator $-\Delta$ with the Dirichlet boundary conditions in the space $\mathbf{B}$. Since $r \geq p, r \geq 2 d$ and $p \in(1,2]$ we infer that $E$ and $\mathbf{B}$ are separable, UMD and type $p$ Banach spaces. Now it is well known that the assumptions of the first and second part of Theorem 3.2 are satisfied by $A_{r}$.

We put $Z=\mathbb{R}$ and $v=v_{\alpha}$. Then, we immediately see that

$$
C_{p}:=\int_{Z}|z|^{p} v(d z)<\infty .
$$

We define a map $\tilde{G}$ by

$$
\tilde{G}(t, \cdot): X \ni u \mapsto\{Z \ni z \mapsto z g \circ u\} \in L^{p}(Z, v ; E), t \in[0, T] .
$$


The map $\tilde{G}$ may not be defined on the whole space $X$, but the map $A_{r}^{-\delta} \tilde{G}$ is because $\delta=\frac{\kappa}{2}$. Indeed, we have the following chain of inequalities.

$$
\begin{aligned}
& \int_{Z}\left|A_{r}^{-\delta} \tilde{G}(u)(z)\right|_{E}^{p} v(d z)=\int_{Z}\left|A_{r}^{-\delta}((g \circ u) z)\right|_{E}^{p} v(d z) \\
& \quad=\int_{Z}\left|A_{r}^{-\delta}(g \circ u)\right|_{E}^{p}|z|^{p} v(d z) \leq C_{p}\left|A_{r}^{\frac{\kappa}{2}} A_{r}^{-\delta}(g \circ u)\right|_{L^{r}}^{p} \leq C_{p}|g|_{L^{\infty}}^{p} .
\end{aligned}
$$

Since the function $g$ is continuous one can easily check that the continuity condition in Assumption 3 is satisfied. Observe that

$$
\tilde{G}(t, u)(z)=G(t, u, z), t \in[0, T], u \in X, z \in Z,
$$

where $G$ is defined in Eq. 4.7.

Since $\kappa>\frac{d}{r}$ we have $E \subset X$. Moreover, it is straightforward to check that the nonlinear map $F$ defined by Eq. 4.6 satisfies Assumption 4 on $X$.

Finally, let $Y=L^{r}(\mathcal{O})$ and $A_{Y}=A_{r}$. Since $1-\frac{q}{q_{\max }}>\frac{1}{2}$ and $\frac{1}{2}>\frac{d}{r}$, we can find $\kappa_{1} \in\left(\frac{d}{r}, \frac{1}{2}\right)$ such that

$$
D\left(A_{r}^{\frac{\kappa_{1}}{2}}\right) \subset X \subset Y .
$$

Thus all the assumptions (with our choice of spaces and maps) of Theorem 3.2 are satisfied and therefore the proof of the existence of a solution with the requested properties follows.

\section{Application II: Reaction-diffusion Equations of an Arbitrary Order with Space-Time Lévy Noise}

The aim of this section is to show how Theorem 3.4 can be applied to Stochastic reactiondiffusion equations driven by the space-time Poissonian white noise. This type of noise, which is a generalization of the space-time white noise, has been treated quite often in the literature, see for instance [65, Definition 7.24] (see also [7]).

We begin by introducing the assumptions on the drift of our problem and the driving noise. In the second subsection we will present the detail about the coefficient of the noise. Finally, after a careful statement of the assumptions on our problem we will formulate and prove the existence of a martingale solution to stochastic reaction-diffusion equations driven by a space-time Poissonian noise.

\subsection{The Noise and the Deterministic Nonlinear Part of the Problem}

Let $d \geq 1, p \in(1,2]$, and $\mathcal{O}$ be a bounded open domain in $\mathbb{R}^{d}$ with boundary $\partial \mathcal{O}$ of $C^{\infty}$ class. We consider a complete filtered probability space $(\bar{\Omega}, \overline{\mathcal{F}}, \overline{\mathbb{F}}, \overline{\mathbb{P}})$ where the filtration $\overline{\mathbb{F}}=\left(\overline{\mathcal{F}}_{t}\right)_{t \geq 0}$ satisfies the usual conditions.

The assumptions on the noise in the equation that we are interested in are given below. 
Assumption 7 Let $\Pi: \bar{\Omega} \rightarrow M_{I}(\mathcal{O} \times[0, \infty))$ be the space-time Poissonian white noise on $(\mathcal{O} \times \mathbb{R}, \mathcal{B}(\mathcal{O}) \times \mathcal{B}(\mathbb{R}) \text {, Leb } \otimes \text { Leb })^{4}$ with intensity measure $v \otimes$ Leb. We assume that:

$\operatorname{supp} v \subset(-1,1)$ and there exists $p \in(1,2]: C_{p}(v):=\int_{\mathbb{R}}|z|^{p} v(d z)<\infty$.

Remark 5.1 By Theorem A.6 we can find a homogeneous Poisson random measure $\eta$ with intensity measure $\hat{v} \otimes$ Leb such that

$$
\begin{aligned}
\eta(\bar{\omega})(U \times C \times D) & =\Pi(\bar{\omega})(U \times C \times D), U \in \mathcal{B}(\mathcal{O}), \quad C \in \mathcal{B}(\mathbb{R}), \quad D \in \mathcal{B}([0, \infty)), \\
\hat{v}(C) & =\int_{\mathbb{R}} \int_{\mathcal{O}} 1_{C}(\zeta, \xi) d \zeta v(d \xi), \quad C \in \mathcal{B}(\mathcal{O}) \times \mathcal{B}(\mathbb{R}) .
\end{aligned}
$$

We fix $p$ as above for the remainder of this section. We also put $\hat{v}=\operatorname{Leb} \otimes v$, where Leb is the Lebesgue measure on $\mathcal{O}$.

Let also $k$ be a positive integer. Borrowing the presentation of [10, Section 6.3] we introduce a differential operator $\mathbf{A}$ of order $2 k$ as follows.

(a) The differential operator $\mathbf{A}$ defined by

$$
\mathbf{A} u(x)=-\sum_{|\alpha| \leq 2 k} a_{\alpha}(x) D^{\alpha} u(x), x \in \mathcal{O},
$$

is properly elliptic (see [73, Section 4.9.1]). The coefficients $a_{\alpha}$ are $C^{\infty}$ functions on the closure $\overline{\mathcal{O}}$ of $\mathcal{O}$.

(b) A system $\mathcal{B}=\left\{\mathcal{B}_{j}\right\}_{j=1}^{k}$ of differential operators on $\partial \mathcal{O}$ is given,

$$
\mathcal{B}_{j}=\sum_{|\alpha| \leq m_{j}} b_{j, \alpha} D^{\alpha}
$$

with the coefficients $b_{j, \alpha}$ being $C^{\infty}$ functions on $\partial \mathcal{O}$. The orders $m_{j}$ of the operators $\mathcal{B}_{j}$ are ordered in the following way:

$$
0 \leq m_{1}<m_{1}<\ldots<m_{k} .
$$

We assume that $m_{k}<2 k$ and

$$
\sum_{|\alpha|=m_{j}} b_{j, \alpha}(\xi) n_{\xi} \neq 0, x \in \mathcal{O}, j=1,2, \ldots, k,
$$

where $n_{\xi}$ is the unit outer normal vector to $\partial \mathcal{O}$ at $\xi \in \partial \mathcal{O}$.

(c) For any $x \in \overline{\mathcal{O}}$ and $\xi \in \mathbb{R}^{n} \backslash\{0\}$ let $a(x, \xi)=\sum_{|\alpha|=2 k} a_{\alpha}(x) \xi^{\alpha}$. We assume that

$$
(-1)^{k} \frac{a(x, \xi)}{|a(x, \xi)|} \neq-1, x \in \overline{\mathcal{O}}, \xi \in \mathbb{R}^{n} \backslash\{0\} .
$$

(d) If $b_{x, \xi}=\sum_{|\alpha|=2 k} a_{\alpha}(x) \xi^{\alpha}$ then for all $x \in \partial \mathcal{O}, \xi \in T_{x}(\partial \mathcal{O}), t \in(-\infty, 0]$ the polynomials

$$
\left\{\tau \rightarrow b_{j}\left(x, \xi+\tau n_{x}\right)\right\}, j=1, \cdots, k
$$

\footnotetext{
${ }^{4}$ Leb is the Lebesgue measure on $\mathcal{O}$ and $\mathbb{R}$ and we refer to Appendix A for the definition and facts about space-time Poissonian white noise.
} 
are linearly independent modulo polynomial $\left\{\tau \rightarrow \prod_{j=1}^{k}\left(\tau-\tau^{+}(t)\right\}\right.$. Here $T_{x}(\mathcal{O})$ is the set of all tangent vectors to $\partial \mathcal{O}$ at $x \in \partial \mathcal{O}$ and $\tau_{j}^{+}(t)$ are the roots with positive imaginary part of the polynomial defined by

$$
\mathbb{C} \ni \tau \rightarrow a\left(x, \xi+\tau n_{x}\right)-t .
$$

The differential operator $\mathbf{A}$ induces a linear unbounded map $A_{r}$ on the Banach space $L^{r}(\mathcal{O})$, $r>1$, defined by

$$
\left\{\begin{array}{l}
D\left(A_{r}\right)=\left\{u \in H^{2 k, r}:\left.\mathcal{B}_{j} u\right|_{\partial \mathcal{O}}=0 \text { for } m_{j}<2 k-\frac{1}{r}\right\}, \\
A_{r} u=\mathbf{A} u, \quad u \in D\left(A_{r}\right) .
\end{array}\right.
$$

Assume $\theta \geq 0, r \in[p, \infty)$. Let $A_{r}$ be the linear operator in the Banach space $L^{r}(\mathcal{O})$ defined in Eq. 5.7. The space $D\left(A_{r}^{\frac{\theta}{2 k}}\right)$ will be used in what follows quite often and hence it is convenient to write it down in terms of Sobolev spaces and boundary conditions, as in equality (5.7). We have, for $\theta \in[0,2 k]$,

$$
D\left(A_{r}^{\frac{\theta}{2 k}}\right)=H_{\mathcal{B}}^{\theta, r}:=\left\{u \in H^{\theta, r}:\left.\mathcal{B}_{j} u\right|_{\partial \mathcal{O}}=0 \text { for } m_{j}<\theta-\frac{1}{r}\right\},
$$

Throughout we put $E=H_{\mathcal{B}}^{\theta, r}$. It is well-known, see for instance Triebel's monograph [73, Section 4.9.1], Seeley's paper [71] or Lunardi's book [53, Section 3.2] (also [10, Section 6.3]), that $A_{r}$ satisfies parts 1(iii)-1(v) of Assumption 1 on the space $E$.

Now we introduce a nonlinear map which will play the role of the drift for our stochastic equation.

Assumption 8 Assume that a function $f:[0, T] \times \mathcal{O} \times \mathbb{R} \rightarrow \mathbb{R}$ is separately continuous and bounded. Moreover, we assume that $f(t, x, \cdot)$ is continuous uniformly w.r.t. $(t, x)$.

We denote by $F$ the Nemytskii map associated to $f$, i.e., defined by

$$
F(t, u)(x):=f(t, x, u(x)), \quad u \in L^{r}(\mathcal{O}), x \in \mathcal{O}, t \in[0, T],
$$

and assume that $F:[0, T] \times L^{r}(\mathcal{O}) \rightarrow L^{r}(\mathcal{O})$.

The restrictions of $F$ to $[0, T] \times H_{\mathcal{B}}^{\theta, r}$ will also be denoted by $F$.

\subsection{Coefficient of the Noise}

We begin this subsection with the precise statement of the assumptions on the coefficient of the noise.

Assumption 9 Let $g:[0, T] \times \mathcal{O} \times \mathbb{R} \rightarrow \mathbb{R}$ be a bounded function that is separately continuous with respect to the first and the second variables, and $g(t, x, \cdot)$ is continuous uniformly w.r.t. $(t, x)$.

We define a nonlinear map $G_{0}:[0, T] \times L^{r}(\mathcal{O}) \rightarrow L^{r}(\mathcal{O})$ to be the Nemytskii operator associated to $g$; that is,

$$
G_{0}(t, u)(x):=g(t, x, u(x)), \quad u \in L^{r}(\mathcal{O}), x \in \mathcal{O} .
$$

Because of Assumption 9, in view of the Lebesgue Dominated Convergence Theorem (DCT) for every $t \geq 0 \quad G_{0}(t, \cdot)$ is a continuous map from $L^{r}(\mathcal{O})$ into itself and for each $u \in L^{r}(\mathcal{O})$, the function $G_{0}(\cdot, u):[0, T] \rightarrow L^{r}(\mathcal{O})$ is strongly measurable. 
Let $B_{r, \infty}^{s}(\mathcal{O})$, be the Besov space as defined in Appendix B. By Proposition B.1 along with Corollary B.4 we can define a bounded linear map

$$
\Phi: L^{p}(\mathcal{O}) \rightarrow L^{p}\left(\mathcal{O} \times \mathbb{R} ; \hat{v} ; B_{r, \infty}^{-\left(d-\frac{d}{r}\right)}(\mathcal{O})\right),
$$

by setting, for $v \in L^{p}(\mathcal{O})$,

$$
[\Phi v](x, y)=\left(v(x) \delta_{x}\right) y, \quad(x, y) \in \mathcal{O} \times \mathbb{R} .
$$

Indeed, $\Phi$ is linear and by Corollary B.4 and Eq. 5.1 we have the following chain of equalities/inequalities

$$
\begin{aligned}
& \int_{\mathcal{O} \times \mathbb{R}}|[\Phi v](x, y)|_{B_{r, \infty}^{-\left(d-\frac{d}{r}\right)}(\mathcal{O})}^{p} d x v(d y)=\int_{\mathcal{O} \times \mathbb{R}}\left|\left(v(x) \delta_{x}\right) y\right|_{B_{r, \infty}^{-\left(d-\frac{d}{r}\right)}(\mathcal{O})}^{p} \hat{v}(d x, d y) \\
= & \int_{\mathcal{O}}\left|v \delta_{x}\right|_{B_{r, \infty}^{-\left(d-\frac{d}{r}\right)}(\mathcal{O})}^{p} d x \times \int_{\mathbb{R}}|y|^{p} v(d y) \leq C C_{p}(v)|v|_{L^{p}(\mathcal{O})}^{p} .
\end{aligned}
$$

Finally, by the choice of $\theta, r$, and $p$ above, the embeddings

$$
H_{\mathcal{B}}^{\theta, r} \subset L^{r}(\mathcal{O}) \subset L^{p}(\mathcal{O}),
$$

are continuous, so we can define a nonlinear map $G$ by

$$
G:=\Phi \circ G_{0}:[0, T] \times C_{0}(\mathcal{O}) \rightarrow L^{p}\left(\mathcal{O} \times \mathbb{R}, \hat{v} ; B_{r, \infty}^{-\left(d-\frac{d}{r}\right)}(\mathcal{O})\right) .
$$

In what follows we will also denote by $G$ the restriction of the previously defined map $G$ to the sets $[0, T] \times H_{\mathcal{B}}^{\theta, r}$ with $r \in(p, \infty)$ and $\theta \geq 0$. It follows from the corresponding properties of the map $G_{0}$ that for every $t \geq 0, G(t, \cdot)$ is continuous and that for each $u \in H_{\mathcal{B}}^{\theta, r}$ the function $G(\cdot, u)$ is strongly measurable.

Claim 5.1 Assume that $p \in(1,2], d \in \mathbb{N}, r \geq p, k \in \mathbb{N}$ and $\theta \geq 0$ satisfy

$$
\theta+d-\frac{d}{r}<\frac{2 k}{p}
$$

Then there exists $\delta<\frac{1}{p}$ such that the map $A_{r}^{-\delta} G$ defined on $[0, T] \times E$ is $L^{p}(Z, v, E)$ valued, bounded and continuous on $E:=H_{\mathcal{B}}^{\theta, r}$ and measurable on $[0, T]$.

Proof Let us fix $k, r, d, \theta$ and $p$ as in the assumptions. Thus, we can choose $\kappa>d-\frac{d}{r}$ such that $\delta:=\frac{\theta+\kappa}{2 k}<\frac{1}{p}$. Let us also notice that $E \subset H^{\theta, r}(\mathcal{O})$. Therefore, since $A_{r}^{-\delta}$ maps $H^{-\kappa, r}(\mathcal{O})$ into $H^{\theta, r}(\mathcal{O})$ and, by [73, Theorem 4.6.1-(a,b)], the Banach space $B_{r, \infty}^{-\left(d-\frac{d}{r}\right)}(\mathcal{O})$ is continuously embedded in $H^{-\kappa, r}(\mathcal{O})$, we infer that the map $A^{-\delta} G$ is $L^{p}(\mathcal{O} \times \mathbb{R}, \hat{v} ; E)$ valued continuous. Therefore, by the continuity and boundedness of the function $g$, the function $A^{-\delta} G$ from $[0, T] \times E$ into $L^{p}(\mathcal{O} \times \mathbb{R}, \hat{v} ; E)$ is separately continuous and bounded. Since $\delta<\frac{1}{p}$ we deduce that $G$ satisfies Assumption 3 with $\rho=\frac{1}{p}-\delta$.

Remark 5.2 If $d<\frac{2 k}{p}$, then we can find $\theta>\frac{d}{r}$ such that condition (5.13) is satisfied and the space $E$ is continuously embedded in $C_{0}(\mathcal{O})$. 


\subsection{The Formulation of the Result}

Let $\tilde{\eta}$ be the compensated Poisson random measure associated to the time-homogeneous Poisson random measure given by Remark 5.1. With the functional setting we described above, the problem that we are interested in is

$$
\begin{aligned}
d u(t)+A_{r} u(t) d t & =\int_{\mathcal{O} \times \mathbb{R}} G(u(t))[\xi, \zeta] \tilde{\eta}(d \xi \times d \zeta \times d t) \\
& +F(t, u(t)) d t, t \in(0, T], \\
u(0) & =u_{0} .
\end{aligned}
$$

Remark 5.3 A very important example of problem (5.14) is the following SPDE

$$
\begin{aligned}
\frac{\partial}{\partial t} u(t, \xi)+\mathbf{A} u(t) d t & =f(u(t, \xi))+g(u(t, \xi))[\dot{L}(\xi, t)] \xi \in \mathcal{O}, t \in(0, T], \\
u(0, \xi) & =u_{0}(\xi), \quad \xi \in \mathcal{O}, \\
u(t, \xi) & =0, \text { for } \xi \in \partial \mathcal{O}, t \in(0, T],
\end{aligned}
$$

where $\mathbf{A}$ is a second order differential operator, both $f$ and $g$ are continuous and bounded real functions defined on $\mathbb{R}$ and, roughly speaking, $\dot{L}$ denotes the Radon-Nikodym derivative of the space-time Lévy white noise ${ }^{5} L$, i.e.,

$$
\dot{L}(\xi, t):=\frac{\partial L(\xi, t)}{\partial t \partial \xi} .
$$

We are finally ready to define the concept of solution to problem (5.14).

Definition 5.4 Let $p \in(1,2]$ and $v$ a Lévy measure on $\mathbb{R}$ satisfying condition (5.1). Let $A_{r}$ be the linear operator in the Banach space $L^{r}(\mathcal{O})$ defined by Eq. 5.7. Put $E=H_{\mathcal{B}}^{\theta, r}$, for some $\theta \geq 0$ and $r \geq p$.

An $E$-valued martingale solution to Eq. 5.14 with càdlàg paths in $L^{r}(\mathcal{O})$ is a system

$$
(\Omega, \mathcal{F}, \mathbb{P}, \mathbb{F}, \eta, u),
$$

where

(i) $(\Omega, \mathcal{F}, \mathbb{F}, \mathbb{P})$ is a complete filtered probability space equipped with a filtration $\mathbb{F}=$ $\left\{\mathcal{F}_{t}\right\}_{t \geq 0}$ satisfying the usual conditions,

(ii) $\eta$ is a space-time Poissonian white noise ${ }^{6}$ on $\mathcal{O} \times \mathbb{R}$ with jump size intensity $\hat{v}=$ Leb $\otimes v$.

(iii) $\quad u$ is a $E$-valued $\mathbb{F}$-progressively measurable stochastic process such that

$$
\mathbb{E} \int_{0}^{T}|u(s)|_{E}^{p} d s<\infty .
$$

(iv) $\quad u$ is a $L^{r}(\mathcal{O})$-valued càdlàg process.

\footnotetext{
${ }^{5}$ We refer again to Appendix A for the definition of space-time Lévy noise.

${ }^{6}$ We refer to Appendix A for the definitions and facts about space-time Lévy and Poissonian noise
} 
(v) for every $t \in[0, T], u$ satisfies the following equation $\mathbb{P}$-a.s.

$$
\begin{aligned}
u(t) & =e^{-t A_{r}} u_{0}+\int_{0}^{t} e^{-(t-r) A_{r}} F(r, u(r)) d r \\
+ & \int_{0}^{t} \int_{\mathcal{O} \times \mathbb{R}} e^{-(t-r) A_{r}} G(u(r))[\xi, \zeta] \tilde{\eta}(d \xi \times d \zeta \times d r) .
\end{aligned}
$$

Remark 5.5 The last condition in Definition 5.4 should be understood that both integrals in equality (5.18) make sense as $E$-valued random variables and (5.18) holds as an equality of $E$-valued random variables.

The following result will be shown by applying Theorem 3.4.

Theorem 5.6 Let $p \in(1,2]$, v be a Lévy measure on $\mathbb{R}$ satisfying condition (5.1), and $\mathbf{A}$ be a differential operator having the properties $(a)-(d)$ in Section 5.1. For $\theta \geq 0$ and $r \geq p$ we put $E=H_{\mathcal{B}}^{\theta, r}$, where $A_{r}$ is the linear operator in the Banach space $L^{r}(\mathcal{O})$ defined in Eq. 5.7. Let $F$ and $G$ be the two maps defined in Eqs. 5.9 and 5.12, respectively, and satisfying Assumptions 8 and 9, see pages 29 and 29. In addition, let us assume that the numbers $p, r, \theta, d$ and $k$ satisfy (5.13). Then, for every $u_{0} \in H_{\mathcal{B}}^{\theta, r}$ there exists a $H_{\mathcal{B}}^{\theta, r}$-valued martingale solution $u$ to Eq. 5.14 with càdlàg trajectories in $L^{r}(\mathcal{O})$.

The above theorem can be reformulated in terms of space-time Lévy noise, but since such a result would not be significantly different from the last one, we omit it and leave as an exercise to an interested reader.

Proof of Theorem 5.6 Let us fix the numbers $d, k, p$, and $r$, the space $E$ and the operator $A_{r}$ as in the statement of the theorem. Also, let $F$ (resp. $G$ ) be defined by equality (5.9) (resp. (5.12)).

Since $r \geq p$, the separable Banach spaces $E$ and $B$ are UMD and martingale type $p$. As we mentioned above $A_{r}$ has the BIP property on $E$, is a positive operator with compact resolvent and $-A_{r}$ generates a contraction type $C_{0}$-semigroup on $E$. Owing to Claim 5.1 we can find $\rho \in\left[0, \frac{1}{p}\right)$ such that the map $A_{r}^{\rho-\frac{1}{p}} G$ defined on $[0, T] \times E$ is $L^{p}(Z, v, E)$-valued, bounded and continuous w.r.t. $E$ and measurable with respect to time. Thus all assumptions but Assumption 5 of Theorem 3.4 are satisfied. However, by Assumption 8 the Nemytskii map $F$ defined for $t \in[0, T]$ and $u \in E$ by

$$
F(t, u)(x):=f(t, x, u(x)), \quad x \in \mathcal{O},
$$

satisfies Assumption 5. Hence, since Theorem 3.4 is applicable, we infer that problem (5.14) has a $E$-valued martingale solution $u$. Since $A_{r}$ is a infinitesimal generator of a contraction $C_{0}$-semigroup on $L^{r}(\mathcal{O})$, by [77], the paths of the process $u$ are càdlàg in $L^{r}(\mathcal{O})$.

Remark 5.7 Let $\mathcal{O}$ be a bounded open domain in $\mathbb{R}^{d}$, with $d \geq 1$. Let $n$ be a fixed natural number. For each $i=1, \cdots, n$ let $v_{i}$ be a Lévy measure on $\mathbb{R}$ satisfying (5.1). For each $i=$ 
$1, \cdots, n$, let $\left\{L_{i}(t) ; t \geq 0\right\}$ be a Lévy noise with Lévy measure $v_{i}$. For a fixed $T \in(0, \infty)$ we consider the following system of SPDEs

$$
\left\{\begin{aligned}
& d u_{i}(t)+\mathbf{A}_{i} u_{i}(t) d t= \sum_{k=1}^{n} g_{i \ell}\left(t, x, u_{1}(t, x), \ldots, u_{n}(t, x)\right) d L_{\ell}(t) \\
& \quad+f_{i}\left(t, x, u_{1}(t, x), \ldots, u_{n}(t, x)\right) d t, t \in(0, T], x \in \mathcal{O}, \\
& u_{i}(0)=u_{i, 0}, x \in \mathcal{O}, \\
& \mathcal{B}_{j, i} u_{i}(t, x)=0, t \in[0, T], x \in \partial \mathcal{O},
\end{aligned}\right.
$$

where $\mathbf{A}_{i}, i=1, \cdots, n$, are differential operators of order $2 k$ satisfying conditions (a)-(d), see pages 27 and 28. Furthermore, we assume that

$$
f=\left[f_{i}\right]_{i=1}^{n}:[0, T] \times \mathcal{O} \times \mathbb{R}^{n} \rightarrow \mathbb{R}^{n}, \quad g=\left[g_{i, \ell}\right]_{i, \ell=1}^{n}:[0, T] \times \mathcal{O} \times \mathbb{R}^{n} \rightarrow \mathbb{R}^{n \times n},
$$

are separately continuous and bounded. In addition, we assume that $g(t, x, \cdot)$ is continuous uniformly w.r.t. $(t, x)$. Problem (5.19) was studied by Cerrai in [20] when each $L_{\ell}$ is a Wiener process.

We will apply the previous theorem on the Banach space $E=H_{\mathcal{B}}^{\theta, r}\left(\mathcal{O}, \mathbb{R}^{n}\right)$ to check that for any $u_{0}=\left(u_{i, 0}\right)_{i=1}^{n} \in H_{0}^{1, r}\left(\mathcal{O}, \mathbb{R}^{n}\right) \cap H_{\mathcal{B}}^{\theta, r}\left(\mathcal{O}, \mathbb{R}^{n}\right)$ there exists a $H_{0}^{1, r}\left(\mathcal{O}, \mathbb{R}^{n}\right) \cap$ $H_{\mathcal{B}}^{\theta, r}\left(\mathcal{O}, \mathbb{R}^{n}\right)$-valued martingale solution to Eq. 5.19 with càdlàg paths in $L^{r}\left(\mathcal{O}, \mathbb{R}^{n}\right)$. For this purpose we consider the diagonal matrix

$$
A=\left(\begin{array}{cccc}
A_{1} & 0 & \ldots & 0 \\
0 & A_{2} & \ldots & 0 \\
\vdots & \vdots & \ddots & \vdots \\
0 & 0 & \ldots & A_{n}
\end{array}\right)
$$

and denote by $G_{0}:[0, T] \times L^{r}\left(\mathcal{O}, \mathbb{R}^{n}\right) \rightarrow L^{r}\left(\mathcal{O}, \mathbb{R}^{n}\right)$ (resp. $F$ ) the Nemytskii operator associated to maps $g$ and respectively $f$. We also set $Z=\mathbb{R}^{n}$ and define the Lévy measure $\hat{v}$ on $\mathcal{O} \times Z$ by $\hat{v}=$ Leb $\otimes\left(v_{1} \otimes \cdots \otimes v_{n}\right)$. As above we can define a bounded linear map $\Phi: E \rightarrow L^{p}\left(\mathcal{O} \times Z ; B_{r, \infty}^{-(d-d / r)}\left(\mathcal{O}, \mathbb{R}^{n}\right)\right)$ by formula (5.11), i.e.,

$$
[\Phi v](x, y)=\left(v(x) \delta_{x}\right) y, \quad(x, y) \in \mathcal{O} \times \mathbb{R}^{n},
$$

and put $G=\Phi \circ G_{0}$. The restriction of the maps $F$ and $G$ to sets $[0, T] \times E$ are still denoted by $F$ and $G$, respectively. We denote by $\eta$ the Poisson random measure with intensity measure $\operatorname{Leb}(d t) \otimes \hat{v}(d x, d z)$ on $[0, T] \times \mathcal{O} \times Z$. Then problem (5.19) can be rewritten in the following form

$$
\left\{\begin{array}{l}
d u \quad+A u d t=F(t, u) d t+\int_{\mathcal{O} \times Z} G(t, u)[x, z] \tilde{\eta}(d x \times d z \times d t), \\
u(0)=u_{0}
\end{array}\right.
$$

The existence result we claimed earlier is now a straightforward consequence of Theorem 5.6.

\section{Application III: Stochastic Evolution Equations with Fractional Generator and Polynomial Nonlinearities}

In this section we will deal with a problem that is similar to the problem from the previous section, but with one important modification. From now on we will assume that the nonlinear term $F$ is of polynomial growth. We put $k=1$ and assume that $\mathbf{A}$ and $\mathcal{B}$ are differential operators satisfying conditions (a)-(d), see page 27 . As in the previous section we fix $r>1$ and denote by $A_{r}$ be the linear operator induced by $\mathbf{A}$ in the Banach space $L^{r}(\mathcal{O})$. 
Let $\gamma \in(0,1]$ and $A_{r}^{\gamma}$ be the fractional power of $A_{r}$. It is well known (see, for instance, [72, Theorem 4.3.3]) that

$$
D\left(A_{r}^{\gamma}\right)=H_{\mathcal{B}}^{2 k \gamma, r}=\left\{u \in H^{2 k \gamma, r}:\left.\mathcal{B}_{j} u\right|_{\partial \mathcal{O}}=0 \text { for } m_{j}<2 k \gamma-\frac{1}{r}\right\},
$$

and for any $\theta \in[0,2 k \gamma]$

$$
D\left(\left(A_{r}^{\gamma}\right)^{\frac{\theta}{2 k \gamma}}\right)=H_{\mathcal{B}}^{\theta, r}=\left\{u \in H^{\theta, r}:\left.\mathcal{B}_{j} u\right|_{\partial \mathcal{O}}=0 \text { for } m_{j}<\theta-\frac{1}{r}\right\} .
$$

We also consider a space-time Poissonian white noise $\Pi$ on $(\mathcal{O} \times \mathbb{R}, \mathcal{B}(\mathcal{O}) \times \mathcal{B}(\mathbb{R}))$ with Lévy measure $v \otimes$ Leb satisfying (5.1) with a fixed number $p \in(1,2]$. As in Remark 5.1 to $\Pi$ we can associate a time-homogeneous Poisson random measure $\eta$ with intensity measure $\tilde{v}=\operatorname{Leb} \otimes v$.

Next, let $g:[0, T] \times \mathbb{R} \times \mathcal{O} \rightarrow \mathbb{R}$ be a bounded, separately continuous w.r.t. to the first and second variables, continuous in the third variable uniformly with respect to the other two. Next, as in previous section we consider the map

$$
G:[0, T] \times L^{r}(\mathcal{O}) \rightarrow L^{p}\left(\mathcal{O} \times \mathbb{R}, \hat{v} ; B_{r, \infty}^{-\left(d-\frac{d}{r}\right)}(\mathcal{O})\right),
$$

defined by

$$
[G(t, u)](x, y)=\left[\left(g(t, u(x), x) \delta_{x}\right] y, u \in L^{r}(\mathcal{O}), \quad(x, y) \in \mathcal{O} \times \mathbb{R} .\right.
$$

We also consider a separately continuous function $f:[0, T] \times \mathcal{O} \times \mathbb{R} \rightarrow \mathbb{R}$ satisfying condition (4.5), for some $q \geq 1$. We denote by $F$ the Nemytskii operator defined by

$$
F(t, u)(x):=f(t, x, u(x)), \quad u \in C_{0}(\mathcal{O}), x \in \mathcal{O}, t \in[0, T] .
$$

We consider the following approximation of the function $f$ by a sequence $\left(f_{n}\right)_{n \in \mathbb{N}}$ of functions defined, for any $t \in[0, T], x \in \mathcal{O}$ and $n \in \mathbb{N}$, by

$$
f_{n}(t, x, u):= \begin{cases}f(t, x, u), & \text { if } u \in[-n, n] \\ f(t, x, n), & \text { if } u \geq n, \\ f(t, x,-n), & \text { if } u \leq-n .\end{cases}
$$

By setting $F_{n}(t, u)(\xi)=f_{n}(t, \xi, u(\xi))$ for $(t, u, \xi) \in[0, T] \times C_{0}(\mathcal{O}) \times \mathcal{O}$ we obtain a sequence $\left(F_{n}\right)_{n \in \mathbb{N}}$ of bounded and separately continuous maps defined on $[0, T] \times C_{0}(\mathcal{O})$ into $C_{0}(\mathcal{O})$ satisfying (3.2) uniformly in $n$, and pointwise converging to $F$ in $C_{0}(\mathcal{O})$. Hence, the nonlinear map $F$ defined by Eq. 6.3 satisfies Assumption 4 with $X=C_{0}(\mathcal{O})$.

Remark 6.1 An example of a real valued function $f$ satisfying the above conditions is

$$
f:[0, \infty) \times \mathcal{O} \times \mathbb{R} \ni(t, \xi, u) \mapsto-|u|^{q} \operatorname{sgn}(u) .
$$

With the various mappings we have introduced above we consider the following SPDEs

$$
\begin{aligned}
& d u(t)+A_{r}^{\gamma} u(t) d t=\int_{\mathcal{O} \times \mathbb{R}} G(t, u(t))[\xi, \zeta] \tilde{\eta}(d \xi \times d \zeta \times d t) \\
& +F(t, u(t)) d t, t \in(0, T] \text {, } \\
& u(0)=u_{0} .
\end{aligned}
$$

Theorem 6.2 Let $v$ be a Lévy measure on $\mathbb{R}$ satisfying the conditions (5.1) with a fixed $p \in(1,2]$. Let us also assume that $\mathbf{A}$ is a differential operator satisfying the properties $(a)$ (d) from page 27. For $\gamma \in(0,1]$, let $A_{r}^{\gamma}$ be the fractional power of the linear operator $A_{r}$ defined in Eq. 5.3. Finally, let $F$ and $G$ be the maps defined in Eqs. 6.3 and 6.2, respectively. 
In addition to the assumptions on $G$ above we also assume that $p d<2 k \gamma$ and that $F$ satisfies Assumption 4.5 with some $q \in(1, p)$. If $r>\max \left\{p, \frac{p d}{p-q}\right\}$, then for any $u_{0} \in$ $C_{0}(\mathcal{O})$ there exists a $C_{0}(\mathcal{O})$-valued martingale solution to Eq. 6.5 with càdlàg trajectories in $L^{r}(\mathcal{O})$.

Before we embark on the proof of this result let us make the following remark.

Remark 6.3 If the intensity measure $v$ of the space-time white noise is finite, then as in the proof of Theorem IV.9.1 in [45] the solution can be written as a concatenation of solutions to the deterministic reaction-diffusion equations on random intervals with the initial data being a measure-valued random variable.

To be more precise, let $\lambda:=\operatorname{Leb}(\mathcal{O}) \times v(\mathbb{R}),\left(\tau_{i}\right)_{i \in \mathbb{N}}$ be a family of independent, exponentially distributed random variables with parameter $\lambda$ and

$$
N(t)=\sum_{n=1}^{\infty} 1_{\left[T_{n}, \infty\right)}(t), \quad t \geq 0,
$$

where $T_{n}=\sum_{i=1}^{n} \tau_{i}, n \in \mathbb{N}$. Let also $\left(Y_{i}\right)_{i \in \mathbb{N}}$ be a family of independent $v / v(\mathbb{R})$ distributed random variables and $\left\{x_{i}: i \in \mathbb{N}\right\}$ be a sequence of independent and uniformly distributed random variables in $\mathcal{O}$. Then, the space-time white noise $\eta$ can be written as follows: for any $A \in \mathcal{B}(\mathcal{O}), B \in \mathcal{B}(\mathbb{R})$ and $I \in \mathcal{B}([0, \infty))$

$$
\eta(A \times B \times I)= \begin{cases}0 & \text { if } N(t)=0, \\ \sum_{i=1}^{N(t)} \delta_{x_{i}, Y_{i}, T_{i}}(A \times B \times I) & \text { if } N(t)>0 .\end{cases}
$$

Using this representation the above SPDEs can be described by a deterministic PDE with initial condition being a measure in the time intervals $\left[T_{n}, T_{n+1}\right)$, i.e., $u$ solves the deterministic PDE

$$
\left\{\begin{aligned}
\frac{\partial}{\partial t} u(t, \xi) & +\mathbf{A} u(t) d t=f(u(t, \xi)) \quad \xi \in \mathcal{O}, t \in\left(T_{n}, T_{n+1}\right), \\
u\left(T_{n}^{+}, \xi\right) & =u\left(T_{n}^{-}\right)+Y_{n} \delta_{x_{n}}, \quad \xi \in \mathcal{O}, \\
u(t, \xi) & =0, \text { for } \xi \in \partial \mathcal{O}, t \in\left(T_{n}, T_{n+1}\right) .
\end{aligned}\right.
$$

It follows that our conditions have to be stronger than the conditions in [8], which is indeed the case. In fact, for $\gamma=1$ we assume that $d<\frac{2}{q}$ which is stronger than $d \leq \frac{2}{q-1}$ imposed by Brezis and Friedman in [8].

Proof of Theorem 6.2 We just give a sketch of the proof because it is very similar to the proofs of Theorem 4.1 and Theorem 5.6. Let us fix the numbers $d, \gamma, p, q$, and $r$ as in the statement of the theorem. We denote by $A_{r}^{\gamma}$ the fractional power of the linear operator $A_{r}$ induced by $-\mathbf{A}$ on the Banach space $\mathbf{B}=L^{r}(\mathcal{O})$. Also, let $F$ be defined by equality (6.3).

By Remark 5.2 we can find $\theta>\frac{d}{r}$ such that $\theta+d-\frac{d}{r}<\frac{2 k \gamma}{p}$ and the Banach space $E=H_{\mathcal{B}}^{\theta, r}$ is continuously embedded in $X:=C_{0}(\mathcal{O})$. Thus, owing to the assumption on $g$ (resp. $f$ ) we can argue as in the proof of Claim 5.1 (resp. Claim 6.3) to prove that the map $G$ (resp. $F$ ) satisfies Assumption 3 (resp. Assumption 4) with $\rho \in\left(0, \frac{1}{p}-\frac{d}{2 k \gamma}\right)$. Since $r \geq p, E$ and $\mathbf{B}$ are separable, UMD and type $p$ Banach spaces. Finally, let $Y=L^{r}(\mathcal{O})$ and $A_{Y}=A_{r}^{\gamma}$. Since $1-\frac{q}{q_{\max }}>1-\frac{q}{p}$ and $r>\frac{p d}{p-q}$, we can find $\kappa_{1} \in\left(\frac{d}{r}, 1-\frac{q}{p}\right)$ such that

$$
H^{\kappa_{1}, r} \subset X \subset Y \text {. }
$$


Since $-A_{r}^{\gamma}$ is an infinitesimal generator of a contraction type $C_{0}$-semigroup on $Y=\mathbf{B}=$ $L^{r}(\mathcal{O})$, all the assumptions of Theorem 3.2 are satisfied by problem (5.14). Hence, we easily conclude the proof of Theorem 6.2 from the applicability of Theorem 3.2.

\section{Some Preliminary Results about Stochastic Convolution}

In this section we will state several results concerning the stochastic convolution process.

\subsection{The Stochastic Convolution}

Let us begin with listing the assumptions we will be using throughout the whole section. We assume that $E$ and $A$ are respectively a Banach space and a linear operator satisfying parts 1(i)-1(v) of Assumption 1. A real number $p \in(1,2]$ satisfies part 1(i) of Assumption 1 and $\rho \in\left(0, \frac{1}{p}\right)$ satisfies Assumption 3 .

We also assume that the following are given: a measurable space $(Z, \mathcal{Z})$, a nonnegative measure $v \in M_{+}(Z)$ on $(Z, \mathcal{Z})$, a filtered probability space $\mathfrak{P}=(\Omega, \mathcal{F}, \mathbb{F}, \mathbb{P})$ such that the right-continuous filtration $\mathbb{F}=\left(\mathcal{F}_{t}\right)_{t \geq 0}$ satisfies the usual conditions, and a timehomogeneous Poisson random measure $\eta$ with Lévy measure $v$. For any progressively measurable process $\xi:[0, \infty) \times \Omega \rightarrow L^{p}(Z, v ; E)$ such that

$$
\mathbb{E} \int_{0}^{T} \int_{Z}|\xi(s, z)|^{p} v(d z) d s<\infty, T>0,
$$

one can define the so called stochastic convolution process by the following formula

$$
\mathfrak{S}(\xi)(t):=\int_{0}^{t} \int_{Z} e^{-(t-s) A} \xi(s, z) \tilde{\eta}(d z, d s), t \geq 0 .
$$

We will frequently use the real interpolation spaces $\left(E, D\left(A^{m}\right)\right)_{\theta, q}=D_{A}^{m}(\theta, q)$, for $\theta \in(0,1), q \in[1, \infty)$ and $m \in \mathbb{N}$, defined by

$$
\begin{aligned}
D_{A}(\theta, q) & :=\left\{x \in E:|x|_{D_{A}(\theta, q)}<\infty\right\}, \\
|x|_{D_{A}(\theta, q)}^{q} & =|x|_{E}^{q}+\int_{0}^{1}\left|t^{1-\theta} A e^{-t A} x\right|^{q} \frac{d t}{t}, x \in E .
\end{aligned}
$$

Let us fix $M>0$ and $T>0$. Throughout this section we denote by $\mathcal{B}_{M}(E)$ the set of all $\mathbb{F}$-progressively measurable processes $\xi$ satisfying

$$
\int_{Z}\left|A^{\rho-\frac{1}{p}} \xi(s, z)\right|_{E}^{p} v(d z) \leq M^{p}, \text { for Leb } \otimes \mathbb{P} \text {-a.e. }(s, \omega) \in[0, T] \times \Omega .
$$

Let us recall the following two important results. A proof of the first one can be found in [13, Theorem 2.1].

Theorem 7.1 For every $\theta \in\left(0,1-\frac{1}{p}\right)$ there exists a constant $C=\hat{C}_{\theta}(E)$ such that for any $D_{A}(\theta, p)$-valued progressively measurable process $\xi$ the following inequality holds for every $T>0$,

$$
\mathbb{E} \int_{0}^{T}|\mathfrak{S}(\xi)(t)|_{D_{A}\left(\theta+\frac{1}{p}, p\right)}^{p} d t \leq C \mathbb{E} \int_{0}^{T} \int_{Z}|\xi(t, z)|_{D_{A}(\theta, p)}^{p} v(d z) d t .
$$

Before proceeding to the statement and the proof of the second result, let us state the following important remark. 
Remark Since, by part 1(v) of Assumption 1, A satisfies the BIP property, it follows from [72, Theorem 1.3.3] and [72, Theorem 1.15.3] that, for $\delta, \theta \in(0,1)$ with $\delta<\theta$, the following embeddings are continuous

$$
D_{A}(\theta, q) \subset D\left(A^{\delta}\right) .
$$

Lemma 7.2 If $\rho^{\prime} \in(0, \rho)$, then there exists a constant $C_{1}>0$ such that

$$
\mathbb{E}\left\|A^{\rho^{\prime}} \mathfrak{S}(\xi)\right\|_{L^{p}(0, T: E)}^{p} \leq C_{1} T^{2-p \delta} M^{p}, \quad \xi \in \mathcal{B}_{M}(E) .
$$

Proof of Lemma 7.2 Let us fix $\rho^{\prime} \in(0, \rho)$ and put $\delta=\rho^{\prime}+\frac{1}{p}-\rho \in\left(0, \frac{1}{p}\right)$. Let us also choose $\xi \in \mathcal{B}_{M}(E)$ and put $u=\mathfrak{S}(\xi)$. Since, by [9], a type $p$ UMD Banach space is a martingale type $p$ Banach space, by Eq. 7.2 and Theorem 2.4 we infer that the stochastic convolution process $\int_{0}^{t} \int_{Z} e^{-(t-s) A} A^{\rho-\frac{1}{p}} \xi(s, z) \tilde{\eta}(d z, d s)$ is well-defined. Moreover, by Theorem 7.1 and Eq. 7.4 it takes values in $L^{p}\left(0, T ; D\left(A^{\delta}\right)\right)$ almost surely. Since

$$
u(t)=A^{\frac{1}{p}-\rho} \int_{0}^{t} \int_{Z} e^{-(t-s) A}\left[A^{\rho-\frac{1}{p}} \xi(s, z)\right] \tilde{\eta}(d z, d s), \quad t \in[0, T],
$$

and $\delta=\rho^{\prime}+\frac{1}{p}-\rho$ we infer that $u$ belongs to $L^{p}\left(0, T ; D\left(A^{\rho \prime}\right)\right)$ almost surely. Furthermore,

$$
\begin{aligned}
\mathbb{E}\left|A^{\rho^{\prime}} u(t)\right|_{E}^{p} & =\mathbb{E}\left|\int_{0}^{t} \int_{Z} A^{\delta} e^{-(t-s) A} A^{\rho-\frac{1}{p}} \xi(s, z) \tilde{\eta}(d z, d s)\right|_{E}^{p} \\
& \leq \mathbb{E} \int_{0}^{t} \int_{Z}\left\|A^{\delta} e^{-(t-s) A}\right\|_{\mathcal{L}(E, E)}^{p}\left|A^{\rho-\frac{1}{p}} \xi(s, z)\right|_{E}^{p} v(d z) d s \\
& \leq C M^{p} \int_{0}^{t} \frac{1}{(t-s)^{p \delta}} d s=C M^{p} \frac{t^{1-p \delta}}{1-p \delta} .
\end{aligned}
$$

Since $p \delta \in(0,1)$, it follows from Eq. 7.5 and the Fubini theorem that

$$
\mathbb{E}\left\|A^{\rho^{\prime}} u\right\|_{L^{p}(0, T: E)}^{p} \leq C M^{p} \frac{T^{2-p \delta}}{(1-p \delta)(2-p \delta)} .
$$

Thus the proof of Lemma 7.2 is complete.

Lemma 7.3 Let the assumptions of Lemma (7.2) hold. Let $\rho^{\prime} \in(0, \rho)$ and put $\mathbf{B}=$ $D\left(A^{\rho^{\prime}-1}\right)$. If $\xi \in \mathcal{B}_{M}(E)$, then

(i) there exists a constant $C_{2}=C_{2}(T)>0$ such that

$$
\mathbb{E} \sup _{0 \leq t \leq T}\left|A^{\rho^{\prime}-1} \mathfrak{S}(\xi)(t)\right|^{p} \leq C_{2} M^{p}
$$

(ii) and the process $u=\mathfrak{S}(\xi)$ admits $a \mathbf{B}$-valued càdlàg modification (which will be still denoted by $\mathfrak{S}(\xi))$.

(iii) If in addition the operator $-A$ generates a contraction type semigroup on the space $D\left(A^{\rho-\frac{1}{p}}\right)$, then the parts $(i)$-(ii) are true for $\mathbf{B}=D\left(A^{\rho-\frac{1}{p}}\right)$.

Proof Let us fix $\xi \in \mathcal{B}_{M}(E)$ and set $u=\mathfrak{S}(\xi)$, and $\psi=A^{\rho-\frac{1}{p}-1} \xi$. By Eq. 7.2, we have

$$
\int_{Z}|A \psi(s, z)|^{p} v(d z)<M^{p} \text {, a.a. } s \in[0, T] .
$$


Hence, it is known, see [77, Lemma 3.3], that the process $v$ defined by

$$
v(t):=\int_{0}^{t} \int_{Z} e^{-(t-s) A} \psi(s, z) \tilde{\eta}(d z, d s), t \in[0, T],
$$

is the unique strong solution to the problem

$$
d v(t)+A v(t) d t=\int_{Z} \psi(t, z) \tilde{\eta}(d z, d t)
$$

with $v(0)=0$, and hence satisfies

$$
v(t)+\int_{0}^{t} A v(s) d s=\int_{0}^{t} \int_{Z} \psi(s, z) \tilde{\eta}(d z, d s), t \in[0, T] .
$$

Let $\delta:=\frac{1}{p}+\rho^{\prime}-\rho<1$ and $\beta:=1-\frac{1}{p}+\rho-\rho^{\prime}>0$. By applying $A^{\delta}$ to both sides of the identity (7.6) and by noticing that $A^{-1} u=A^{\frac{1}{p}-\rho} v$, we infer that for $t \in[0, T]$

$$
A^{\rho^{\prime}-1} u(t)=\int_{0}^{t} A^{\rho^{\prime}} u(s) d s+A^{-\beta} \int_{0}^{t} \int_{Z} A^{\rho-\frac{1}{p}} \xi(s, z) \tilde{\eta}(d z, d s) .
$$

Using the inequality (2.14) in Theorem 2.4 we obtain

$$
\begin{aligned}
\mathbb{E} \sup _{0 \leq t \leq T}\left|A^{-\beta} \int_{0}^{t} \int_{Z} A^{\rho-\frac{1}{p}} \xi(s, z) \tilde{\eta}(d z, d s)\right|^{p} \\
\leq C\left\|A^{-\beta}\right\|_{\mathcal{L}(E)} \mathbb{E} \int_{0}^{T} \int_{Z}\left|A^{\rho-\frac{1}{p}} \xi(s, z)\right|^{p} v(d z) d s \leq C T M^{p} .
\end{aligned}
$$

Next applying Hölder's inequality twice and invoking inequality (7.5) we get

$$
\mathbb{E} \sup _{0 \leq t \leq T}\left|\int_{0}^{t} A^{\rho^{\prime}} u(s) d s\right|^{p} \leq T^{p-1} \mathbb{E} \int_{0}^{T}\left|A^{\rho^{\prime}} u(t)\right|^{p} d t \leq C_{1} T^{p} M^{p} .
$$

This completes the proof of (i) with $C_{2}(T)=C C_{21} T+C_{1} T^{p}$.

Since, by [76, Theorem 4.1], the function

$$
\phi: C([0, T] ; E) \times \mathbb{D}([0, T] ; E) \ni(x, y) \mapsto x+y \in \mathbb{D}([0, T] ; E),
$$

is continuous, in view of the identity (7.7) and Theorem 2.4 we easily deduce that the process $u$ has a $D\left(A^{\rho-1}\right)$-valued càdlàg modification. This completes the proof of part (ii) of our lemma.

Since, by [9], any UMD Banach space of type $p$ is also an martingale type $p$ Banach space, and $\xi$ satisfies (7.2), part (iii) is easily deduced by applying [77, Corollary 5.1].

The next lemma is about estimates of $\mathfrak{S}(\xi), \xi \in \mathcal{B}_{M}(E)$ in the Besov-Slobodetskii spaces $W^{\alpha, p}(0, T ; E)$, see its definition on page 5 .

Lemma 7.4 Let the assumptions of parts (i)-(ii) of Lemma 7.3 hold. Assume that $\alpha \in(0, \rho)$. Then there exists a number $C_{3}>0$ such that

$$
\mathbb{E}\|\mathfrak{S}(\xi)\|_{W^{\alpha, p}(0, T ; E)}^{p} \leq C_{3} M^{p}, \quad \xi \in \mathcal{B}_{M}(E) .
$$

Proof Let us fix $\xi \in \mathcal{B}_{M}(E)$ and put $u=\mathfrak{S}(\xi)$. Let us fix $\alpha \in(0, \rho)$ and let us choose an auxiliary $\rho^{\prime} \in(\alpha, \rho)$. In view of Lemma 7.2 and the definition of the (2.3) it is sufficient 
to estimate the mathematical expectation of the seminorm (2.3) of $u$. For this aim, without loss of generality, we can take $s<t \in[0, T]$. As in the Gaussian case we have

$$
u(t)-u(s)=\tilde{S}_{1}(t, s)+\tilde{S}_{2}(t, s),
$$

where

$$
\tilde{S}_{1}(t, s)=\int_{s}^{t} \int_{Z} e^{-(t-r) A} \xi(r ; z) \tilde{\eta}(d z ; d r), \tilde{S}_{2}(t, s)=\left(e^{-(t-s) A}-I\right) u(s) .
$$

In view of the definition (2.3) it is sufficient to prove that there exist two positive numbers $C_{31}, C_{32}$ such that

$$
\begin{aligned}
& S_{1}:=\mathbb{E} \int_{0}^{T} \int_{0}^{t} \frac{\left|\tilde{S}_{1}(t, s)\right|^{p}}{|t-s|^{\alpha p+1}} d s d t \leq C_{31} M^{p}, \\
& S_{2}:=\mathbb{E} \int_{0}^{T} \int_{0}^{t} \frac{\left|\tilde{S}_{2}(t, s)\right|^{p}}{|t-s|^{1+\alpha p}} d s d t \leq C_{32} M^{p} .
\end{aligned}
$$

Let us begin with estimating $S_{1}$. By using the Fubini Theorem, [13, Corollary C.2], the estimate $\left\|A^{\frac{1}{p}-\rho} e^{-(t-r) A}\right\|_{\mathcal{L}(E)}^{p} \leq C(t-r)^{-p\left(\frac{1}{p}-\rho\right)}$ and the definition (7.2) of the class $\mathcal{B}_{M}(E)$, we infer that

$$
\begin{aligned}
S_{1} & \leq C \int_{0}^{T} \int_{0}^{t} \frac{d s d t}{|t-s|^{1+\alpha p}} \times \\
& \mathbb{E} \int_{s}^{t} \int_{Z}\left\|A^{\frac{1}{p}-\rho} e^{-(t-r) A}\right\|_{\mathcal{L}(E)}^{p}\left|A^{\rho-\frac{1}{p}} \xi(r, z)\right|^{p} \nu(d z) d r \\
& \leq C M^{p} \int_{0}^{T} \int_{0}^{t} \frac{d s d t}{|t-s|^{1+\alpha p}} \int_{0}^{t-s} r^{p \rho-1} d r \\
& \leq C M^{p} \int_{0}^{T} \int_{0}^{t} \frac{d s d t}{|t-s|^{1+(\alpha-\rho) p}} \leq C M^{p} T^{1+p(\rho-\alpha)} .
\end{aligned}
$$

In order to study the term $S_{2}$ let us recall, see [63, Theorem II.6.13], that there exists a $C>0$ such that

$$
\left|A^{-\gamma}\left(e^{-h A}-I\right)\right|_{\mathcal{L}(E)} \leq C h^{\gamma}, \quad h>0 .
$$

Therefore, by applying the Young inequality for convolutions we infer that

$$
\begin{aligned}
S_{2} & \leq C \mathbb{E} \int_{0}^{T} \int_{0}^{t} \frac{\left\|A^{-\rho^{\prime}}\left(e^{-(t-s) A}-I\right)\right\|_{\mathcal{L}(E)}^{p}\left|A^{\rho^{\prime}} u(s)\right|^{p}}{|t-s|^{1+p \alpha}} d s d t \\
& \leq C \mathbb{E} \int_{0}^{T}\left|A^{\rho^{\prime}} u(s)\right|^{p}\left(\int_{0}^{T-s} \frac{\left\|A^{-\rho^{\prime}}\left(e^{-\tau A}-I\right)\right\|_{\mathcal{L}(E)}^{p}}{|\tau|^{1+p \alpha}} d \tau\right) d s \\
& \leq C \mathbb{E}\left\|A^{\rho^{\prime}} u\right\|_{L^{p}(0, T ; E)}^{p} \int_{0}^{T} \tau^{-1+p\left(\rho^{\prime}-\alpha\right)} d \tau \\
& \leq C \mathbb{E}\left\|A^{\rho^{\prime}} u\right\|_{L^{p}(0, T ; E)}^{p} T^{p\left(\rho^{\prime}-\alpha\right)} .
\end{aligned}
$$

Invoking Lemma 7.2 and the estimate for $S_{1}$ concludes the proof of the lemma.

Remark 7.5 Since $\alpha \in\left(0, \frac{1}{p}\right)$ we cannot infer from the above Lemma that the process $\mathfrak{S}(\xi)$, $\xi \in \mathcal{B}_{M}(E)$, has an $E$-valued càdlàg modification. It is known, see for instance [48], that if $E$ is a Hilbert space, the driving Lévy process $L$ lives in $E$ and $\left\{e^{-t A}: t \geq 0\right\}$ is contraction type $C_{0}$-semigroup on $E$, then the stochastic convolution process $\int_{0}^{\cdot} e^{-A(\cdot-s)} d L(s)$ 
has an $E$-valued càdlàg modification. If $E$ is a Banach space, then it is sufficient to assume that either $E$ is a $p$-smooth Banach space or the semigroup $\left\{e^{-t A} ; t \geq 0\right\}$ on $E$ is analytic, see [77]. However, we should note that in our framework we do not have such a nice situation. Indeed, roughly speaking, our semigroup is analytic and contractive on a martingale type $p, p \in(1,2]$, Banach space $E$ and our noise lives in a larger space than $E$ (say $D\left(A^{-\alpha}\right), \alpha>0$ ), and in general even if $-A$ is the infinitesimal generator of an analytic semigroup of contraction type it is not known whether the stochastic convolution $\mathfrak{S}(\xi)$, $\xi \in \mathcal{M}^{p}\left(0, T, L^{p}(Z, v ; E)\right)$, has a càdlàg modification in $E$ or in a smaller space, say $D\left(A^{\gamma}\right), \gamma>0$, than $E$. This is even an open question for the case when $E$ is a Hilbert space, see for instance [66].

The next three lemmata are about tightness of the family of the laws of $\left\{\mathfrak{S}(\xi): \xi \in \mathcal{B}_{M}(E)\right\}$

Lemma 7.6 Let the assumptions of parts (i)-(ii) of Lemma 7.3 hold. Then, the family of the laws of $\left\{\mathfrak{S}(\xi): \xi \in \mathcal{B}_{M}(E)\right\}$ is tight on $L^{p}(0, T ; E)$.

Proof As in Lemma 7.3 we choose an auxiliary $\rho^{\prime} \in(0, \rho)$ and put $\mathbf{B}=D\left(A^{\rho^{\prime}-1}\right)$. Let us also put $Y=D\left(A^{\rho^{\prime}}\right)$. Since, by Assumption 1, $A$ has compact resolvent, it follows from the combination of [35, Proposition 5.8], [73, Theorem 1.15.3, pp 103] and [73, Theorem 1.16.4-2, pp 117] that the embeddings $Y \hookrightarrow E$ and $E \hookrightarrow \mathbf{B}$ are compact. Thanks to Lemma 7.2 and Lemma $7.4\left\{\mathfrak{S}(\xi): \xi \in \mathcal{B}_{M}(E)\right\}$ is uniformly bounded on $\mathcal{M}^{p}(0, T ; Y) \cap$ $L^{p}\left(\Omega ; W^{\alpha, p}(0, T ; E)\right)$. Hence, since the embedding

$$
W^{\alpha, p}(0, T ; E) \cap L^{p}\left(0, T: D\left(A^{\rho^{\prime}}\right)\right) \hookrightarrow L^{p}(0, T ; E)
$$

is compact, see [40, Step 1 of Proof of Theorem 2.1], it follows from the Chebyshev inequality and [40, Theorem 2.1] that the laws of $\left\{\mathfrak{S}(\xi): \xi \in \mathcal{B}_{M}(E)\right\}$ are tight on $L^{p}(0, T ; E)$.

Lemma 7.7 Let the assumptions of parts (i)-(ii) of Lemma 7.3 hold. Then the family of the laws of $\left\{\mathfrak{S}(\xi): \xi \in \mathcal{B}_{M}(E)\right\}$ is tight on $\mathbb{D}\left([0, T] ; D\left(A^{\rho^{\prime}-1}\right)\right)$ for any $\rho^{\prime} \in(0, \rho)$.

For the proof of this lemma we need the following general result.

Lemma 7.8 Assume that $p \in(1,2], T>0$. Assume that $E$ and $Y$ are two martingale type $p$ Banach spaces such that the embedding $E \hookrightarrow Y$ is compact. For every $\xi \in \mathcal{B}_{M}(E)$ let a process $v=\Psi(\xi)$ be defined by

$$
v(t)=\int_{0}^{t} \int_{Z} \xi(s, z) \tilde{\eta}(d z, d s), \quad t \in[0, T] .
$$

Then the family of the laws of $\left\{v=\Psi(\xi): \xi \in \mathcal{B}_{M}(E)\right\}$ on $\mathbb{D}([0, T] ; Y)$ is tight.

Proof We need to check items (a) and (b) of Corollary D.2.

By the maximal inequality (2.16) in Corollary 2.6 (see also [13, Corollary C2]) there exists $C>0$ such that for any $\xi \in \mathcal{B}_{M}(E)$ we have

$$
\mathbb{E} \sup _{s \in[0, T]}|v(s)|^{p} \leq C M^{p} .
$$


Let $\varepsilon>0$ and $K_{\varepsilon}=\left\{y \in X:|y| \leq\left(C \varepsilon^{-1}\right)^{\frac{1}{p}} M\right\}$. It follows easily from the Chebyshev inequality that

$$
\mathbb{P}\left(v(t) \notin K_{\varepsilon}, t \in[0, T]\right) \leq\left[\left(C \varepsilon^{-1}\right)^{\frac{1}{p}} M\right]^{-p} \mathbb{E} \sup _{t \in[0, T]}|v(t)|^{p} \leq \varepsilon .
$$

Corollary D.2-(a) follows from this inequality and the compactness of the embedding $E \subset$ $Y$.

Next, let us fix $0 \leq \sigma \leq \tau \leq T$. Then by [13, Corollary C.2] and the Jensen inequality

$$
\begin{aligned}
\mathbb{E} \sup _{t \in[\sigma, \tau]}|v(t)-v(\sigma)|_{Y} & =\mathbb{E} \sup _{t \in[\sigma, \tau]}\left|\int_{\sigma}^{t} \int_{Z} \xi(s, z) \tilde{\eta}(d z, d s)\right|_{Y} \\
& \leq C \mathbb{E}\left(\int_{\sigma}^{\tau} \int_{Z}|\xi(s, z)|_{Y}^{p} v(d z) d s\right)^{\frac{1}{p}} \\
& \leq C\left(\mathbb{E} \int_{\sigma}^{\tau} \int_{Z}|\xi(s, z)|_{Y}^{p} v(d z) d s\right)^{\frac{1}{p}} \leq C M(\tau-\sigma)^{\frac{1}{p}} .
\end{aligned}
$$

Thus we can apply Corollary D.2-(b) from which the sought result follows.

Proof of Lemma 7.7 Let us fix $\xi \in \mathcal{B}_{M}(E)$ and put $u=\mathfrak{S}(\xi)$. Let us fix auxiliary numbers $\rho^{\prime} \in(0, \rho)$ and $\gamma \in\left(\rho^{\prime}, \rho\right)$. Let us put $\beta=\rho-\rho^{\prime}+1-\frac{1}{p}>0$ and let us rewrite identity (7.7) as follows

$$
\begin{aligned}
A^{\rho^{\prime}-1} u(t) & =A^{\rho^{\prime}-\gamma} \int_{0}^{t} A^{\gamma} u(s) d s+A^{-\beta} \int_{0}^{t} \int_{Z} A^{\rho-\frac{1}{p}} \xi(s, z) \tilde{\eta}(d z, d s) \\
& =v_{1}(t)+v_{2}(t), \quad t \in[0, T] .
\end{aligned}
$$

It follows from Lemma 7.8 that the family of laws of $\left\{v_{2}(\xi): \xi \in \mathcal{B}_{M}(E)\right\}$ is tight on $\mathbb{D}([0, T] ; E)$.

On the other hand, since $\gamma<\rho$, by Lemma 7.2 there exists $C>0$ such that

$$
\mathbb{E} \int_{0}^{t}\left|A^{\gamma} u(s)\right|^{p} d s \leq C M^{p} .
$$

Since $\rho^{\prime}-\gamma<0$, the map $A^{\rho^{\prime}-\gamma}: E \rightarrow E$ is compact. Therefore, since $v_{1}(t)=$ $A^{\rho^{\prime}-\gamma} \int_{0}^{t} A^{\gamma} u(s) d s$ we infer that the family of laws of $\left\{v_{1}(\xi): \xi \in \mathcal{B}_{M}(E)\right\}$ are tight on $C([0, T] ; E)$. Hence we easily conclude the proof of (i) since, by [76, Theorem 4.1], the function

$$
\phi: C([0, T] ; E) \times \mathbb{D}([0, T] ; E) \ni(x, y) \mapsto x+y \in \mathbb{D}([0, T] ; E),
$$

is continuous.

We also need the following auxiliary result.

Lemma 7.9 Assume that all the assumptions in Lemma 7.3 are satisfied. Then, for every $q \in\left(p, \frac{1}{\frac{1}{p}-\rho}\right)=\left(p, \frac{p}{1-p \rho}\right)$ and every $r \in(1, p)$ there exists $C>0$ such that

$$
\mathbb{E}|\mathfrak{S}(\xi)|_{L^{q}(0, T ; E)}^{r} \leq C M^{r}, \quad \xi \in \mathcal{B}_{M}(E) .
$$

Moreover, the family of the laws of $\left\{\mathfrak{S}(\xi): \xi \in \mathcal{B}_{M}(E)\right\}$ on $L^{q}(0, T ; E)$ is tight. 
Proof of Lemma 7.9 Let us fix $q \in\left(p, \frac{1}{\frac{1}{p}-\rho}\right)$. Since $q\left(\frac{1}{p}-\rho\right)<1$ and $q>p$ we can find $\rho^{\prime} \in(0, \rho)$ such that $q\left(\frac{1}{p}-\rho\right)=1-p\left(\rho-\rho^{\prime}\right)$. Let us next put

$$
\theta:=\frac{\frac{1}{p}-\rho}{\frac{1}{p}-\rho+\rho^{\prime}}=\frac{1-p \rho}{1-p \rho+p \rho^{\prime}}=\frac{p}{q} \in(0,1),
$$

and let us define, as in proof of Lemma 7.6, $Y=D\left(A^{\rho^{\prime}}\right)$. We also put $\mathbf{B}=D\left(A^{\rho-\frac{1}{p}}\right)$. Then by the reiteration property of the complex interpolation we have,

$$
E=[\mathbf{B}, Y]_{\theta},
$$

and therefore we get, see [6],

$$
|y| \leq|y|_{\mathbf{B}}^{1-\theta}|y|_{Y}^{\theta}, \quad y \in Y .
$$

Next, let us take an arbitrary $r \in(1, p)$ and put $s=\frac{q}{r}, \frac{1}{s}+\frac{1}{s^{*}}=1$. Then $\operatorname{sr} \theta=p$ and, since $r<p, r s^{*}(1-\theta)<p$. Let us choose an auxiliary $\delta>1$ such that $\delta r s^{*}(1-\theta)=p$.

Let us fix $\xi \in \mathcal{B}_{M}(E)$ and put $u=\mathfrak{S}(\xi)$. Then, by the Hölder and Jensen inequalities,

$$
\begin{aligned}
\mathbb{E}|u|_{L^{q}(0, T ; E)}^{r} & \leq \mathbb{E}\left[|u|_{L^{q^{\theta}(0, T ; Y)}}^{r \theta}|u|_{L^{\infty}(0, T ; \mathbf{B})}^{r(1-\theta)}\right]=\mathbb{E}\left[|u|_{L^{p}(0, T ; Y)}^{r \theta}|u|_{L^{\infty}(0, T ; \mathbf{B})}^{r(1-\theta)}\right] \\
& \leq \mathbb{E}\left[|u|_{L^{p}(0, T ; Y)}^{r r \theta}\right]^{\frac{1}{s}} \mathbb{E}\left[|u|_{L^{\infty}(0, T ; \mathbf{B})}^{\delta r s^{*}(1-\theta)}\right]^{\frac{1}{\delta s^{*}}} \\
& =\mathbb{E}\left[|u|_{L^{p}(0, T ; Y)}^{p}\right]^{\frac{r}{q}} \mathbb{E}\left[|u|_{L^{\infty}(0, T ; \mathbf{B})}^{p}\right]^{\frac{1}{\delta}-\frac{r}{\delta q}} \leq C_{4} M^{r},
\end{aligned}
$$

where Lemma 7.2 and Lemma 7.3-(iii) were used to obtain the last inequality. The proof of the first part is complete.

To prove the second part we observe that by the same argument as above, given $q$ and $r$, we can find $\varepsilon>0$ and $C>0$ such that

$$
\mathbb{E}|\mathfrak{S}(\xi)|_{L^{q}\left(0, T ; D\left(A^{\varepsilon}\right)\right)}^{r} \leq C M^{r}, \quad \xi \in \mathcal{B}_{M}(E) .
$$

Moreover, by Lemma 7.4, for any fixed $\alpha \in(0, \rho)$, we can find $C_{3}>0$ such that

$$
\mathbb{E}\|\mathfrak{S}(\xi)\|_{W^{\alpha, p}(0, T ; E)}^{p} \leq C_{3} M^{p}, \quad \xi \in \mathcal{B}_{M}(E) .
$$

Since the embedding $D\left(A^{\varepsilon}\right) \hookrightarrow E$ is compact, the embedding

$$
L^{q}\left(0, T ; D\left(A^{\varepsilon}\right)\right) \cap W^{\alpha, p}(0, T ; E) \hookrightarrow L^{q}(0, T ; E)
$$

is compact. Hence the second part of the Lemma follows.

Next we will formulate an analogous result in the case when only the assumptions of parts (i) and (ii) of Lemma 7.3 are satisfied. The proof will be similar to the last lemma with the difference that instead of taking $\mathbf{B}=D\left(A^{\rho-\frac{1}{p}}\right)$ we need to take $\mathbf{B}=D\left(A^{\rho^{\prime}-1}\right)$.

Lemma 7.10 Let the assumptions of parts (i) and (ii) of Lemma 7.3 be satisfied with $\rho^{\prime} \in$ $(0, \rho)$. Then, for every $q \in\left(p, \frac{p}{1-\rho^{\prime}}\right)$ and every $r \in(1, p)$, there exists $C>0$ such that

$$
\mathbb{E}|\mathfrak{S}(\xi)|_{L^{q}(0, T ; E)}^{r} \leq C M^{r}, \quad \xi \in \mathcal{B}_{M}(E) .
$$

Moreover, the family of the laws of $\left\{\mathfrak{S}(\xi): \xi \in \mathcal{B}_{M}(E)\right\}$ on $L^{q}(0, T ; E)$ is tight. 
Proof of Lemma 10 Let $q \in\left(p, \frac{p}{1-\rho^{\prime}}\right), \rho^{\prime} \in(0, \rho)$ and define $\theta=\frac{p}{q}$. As in proof of Lemma 7.9 we let $Y=D\left(A^{\rho^{\prime}}\right)$ and $\mathbf{B}=D\left(A^{\rho^{\prime}-1}\right)$. Then by the reiteration property of the complex interpolation we have the continuous embedding,

$$
[\mathbf{B}, Y]_{\theta}=D\left(A^{\theta+\rho^{\prime}-1}\right) \subset E .
$$

Owing to Lemma 7.2 and parts (i) and (ii) of Lemma 7.3 we can argue exactly as in the proof of Lemma 7.9 and show that for any $r \in(1, p)$ we have

$$
\mathbb{E}|\mathfrak{S}(\xi)|_{L^{q}\left(0, T ; D\left(A^{\theta+\rho^{\prime}-1}\right)\right)}^{r} \leq C M^{r}, \quad \xi \in \mathcal{B}_{M}(E),
$$

which implies inequality (7.11). Thanks to Eq. 7.12 we can again use the same argument as in proof of Lemma 7.9 to deduce that the family of the laws of $\left\{\mathfrak{S}(\xi): \xi \in \mathcal{B}_{M}(E)\right\}$ on $L^{q}(0, T ; E)$ is tight.

\section{Proof of Theorem 3.4}

We begin the proof of Theorem 3.4 by introducing a sequence of approximating processes. Let us fix for the whole section a number $T>0$. Consider a sequence $\left(x_{n}\right)_{n \in \mathbb{N}} \subset E$ such that $x_{n} \rightarrow u_{0}$ strongly in $D\left(A^{\rho-\frac{1}{p}}\right)$ as $n \rightarrow \infty$. Define a function $\phi_{n}:[0, \infty) \rightarrow[0, \infty)$ by $\phi_{n}(s)=\frac{k}{2^{n}}$, if $k \in \mathbb{N}$ and $\frac{k}{2^{n}} \leq s<\frac{k+1}{2^{n}}$, i.e., $\phi_{n}(s)=2^{-n}\left[2^{n} s\right], s \geq 0$, where [t] is the integer part of $t \in \mathbb{R}$. Let us define a sequence $\left(u_{n}\right)_{n \in \mathbb{N}}$ of adapted $E$-valued processes by

$$
\begin{aligned}
u_{n}(t)= & e^{-t A} x_{n}+\int_{0}^{t} e^{-(t-s) A} F\left(s, \hat{u}_{n}(s)\right) d s \\
& +\int_{0}^{t} \int_{Z} e^{-(t-s) A} G\left(s, \hat{u}_{n}(s) ; z\right) \tilde{\eta}(d z ; d s), \quad t \in[0, T],
\end{aligned}
$$

where $\hat{u}_{n}$ is defined by

$$
\hat{u}_{n}(s):= \begin{cases}x_{n}, & \text { if } s \in\left[0,2^{-n}\right), \\ f_{\phi_{n}(s)-2^{-n}}^{\phi_{n}(s)} u_{n}(r) d r, & \text { if } s \geq 2^{-n},\end{cases}
$$

and where we have used the following shorthand notation

$$
f_{A} f(t) d t:=\frac{1}{\operatorname{Leb}(A)} \int_{A} f(t) d t, \quad A \in \mathcal{B}([0, T])
$$

(Here Leb denotes the Lebesgue measure.) The $E$-valued process $\hat{u}$ is piecewise constant adapted and hence progressively measurable. Between the grid points, Eq. 8.1 is linear, therefore, $u_{n}$ is well defined for all $n \in \mathbb{N}$.

Next we will prove certain uniform estimates for the sequence $\left(u_{n}\right)_{n \in \mathbb{N}}$. Let us recall that $F$ is a bounded nonlinear map defined on $[0, T] \times E$ and taking values in $D\left(A^{\rho-1}\right)$. Furthermore, it is separately continuous. 
Proposition 8.1 For any $\alpha \in(0, \rho)$ and $\rho^{\prime} \in(0, \rho)$, there exists a constant $C$ such that the following inequalities hold

$$
\begin{aligned}
& \sup _{n \in \mathbb{N}} \mathbb{E}\left\|A^{\rho^{\prime}} u_{n}\right\|_{L^{p}(0, T ; E)}^{p} \leq C, \\
& \sup _{n \in \mathbb{N}} \mathbb{E}\left\|A^{\rho^{\prime}} \hat{u}_{n}\right\|_{L^{p}(0, T ; E)}^{p} \leq C, \\
& \sup _{n \in \mathbb{N}} \mathbb{E}\left\|u_{n}\right\|_{W \alpha, p(0, T ; E)}^{p} \leq C .
\end{aligned}
$$

Proof Without loss of generality we take $T=1$. For each $n \in \mathbb{N}$ we divide the interval $[0,1]$ into small intervals of length $2^{-n}$ each by setting: $I_{k}=\left[\frac{k}{2^{n}}, \frac{k+1}{2^{n}}\right), k=0, \ldots, 2^{n}-1$. We also put $J_{0}=I_{0}$ and $J_{k}=\bigcup_{\ell=1}^{k} I_{\ell}, k=1, \ldots, 2^{n}-1$. Define the sequences of processes $\left\{u_{n}^{k}: k=0, \ldots, 2^{n}-1\right\}$ and $\left\{\hat{u}_{n}^{k}: k=0, \ldots, 2^{n}-1\right\}$ inductively by

$$
\begin{aligned}
& \left\{\begin{aligned}
u_{n}^{0}(t)= & e^{-t A} x_{n}+\int_{0}^{t} e^{-(t-s) A} F\left(s, x_{n}\right) d s \\
& \quad+\int_{0}^{t} \int_{Z} e^{-(t-s) A} G\left(s, x_{n}\right) \tilde{\eta}(d z, d s), \quad t \in I_{0}, \\
\hat{u}_{n}^{0}(t)= & x_{n}, \quad t \in I_{0}
\end{aligned}\right.
\end{aligned}
$$

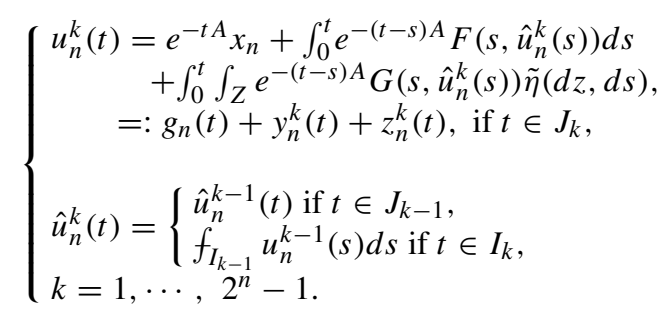

Note that, by definition, $u_{n}^{k}$ is equal to the restriction of $u_{n}$ to $J_{k}$ and $u_{n}=u_{n}^{2^{n}-1}$. Hence to prove our proposition it is sufficient to check that the estimates (8.3)-(8.5) are true and uniform w.r.t $k$ on $J_{k}$ for $u_{n}^{k}$ and $\hat{u}_{n}^{k}$ with $k=0, \ldots, 2^{n}-1$.

On the interval $J_{0}$ we have

$$
\begin{aligned}
& u_{n}^{0}(t)=e^{-t A} x_{n}+\int_{0}^{t} e^{-(t-s) A} F\left(s, x_{n}\right) d s \\
& +\int_{0}^{t} \int_{Z} e^{-(t-s) A} G\left(s, x_{n}\right) \tilde{\eta}(d z, d s) \\
& =g_{n}(t)+y_{n}^{0}(t)+z_{n}^{0}(t) .
\end{aligned}
$$

First, it follows from [28, Theorems 2 and 7] that there exists a constant $C>0$ such that for any $n \in \mathbb{N}$

$$
\mathbb{E}\left[\left\|A^{\rho} g_{n}\right\|_{L^{p}\left(J_{0} ; E\right)}^{p}+\left\|A^{\rho} y_{n}^{0}\right\|_{L^{p}\left(J_{0} ; E\right)}^{p}\right] \leq C\left|x_{n}\right|^{p}+C \mathbb{E}\left\|A^{\rho-1} F\right\|_{L^{p}\left(J_{0} ; E\right)}^{p} \leq C .
$$

Secondly, we derive from [28, Theorems 7 and 19] that for any $\alpha \in(0, \rho)$ there exists a constant $C>0$ such that for any $n \in \mathbb{N}$

$$
\mathbb{E}\left[\left\|g_{n}\right\|_{W^{\alpha, p}\left(J_{0} ; E\right)}^{p}+\left\|y_{n}^{0}\right\|_{W^{\alpha, p}\left(J_{0} ; E\right)}^{p}\right] \leq C\left|x_{n}\right|^{p}+C \mathbb{E}\left\|A^{\rho-1} F\right\|_{L^{p}\left(J_{0} ; E\right)}^{p} \leq C .
$$

Hence combining these two remarks with Lemma 7.2, Lemma 7.4 and Proposition E.1 we infer that (8.3)-(8.4) are true on $J_{0}$ for $u_{n}^{0}$ and $\hat{u}_{n}^{0}$. Using the same approach we can prove 
by induction that for each $\alpha$ and $\rho$ as above there exists a constant $C>0$ such that for any $n \in \mathbb{N}$ and $k \in\left\{0, \ldots, 2^{n}-1\right\}$ we have

$$
\begin{aligned}
& \mathbb{E}\left[\left\|A^{\rho} g_{n}\right\|_{L^{p}\left(J_{k} ; E\right)}^{p}+\left\|A^{\rho} y_{n}^{k}\right\|_{L^{p}\left(J_{k} ; E\right)}^{p}\right] \leq C\left|x_{n}\right|^{p}+C \mathbb{E}\left\|A^{\rho-1} F\left(., \hat{u}_{n}^{k}\right)\right\|_{L^{p}\left(J_{k} ; E\right)}^{p} \\
& \leq C,
\end{aligned}
$$

and

$$
\begin{aligned}
& \mathbb{E}\left[\left\|g_{n}\right\|_{W^{\alpha, p}\left(J_{k} ; E\right)}^{p}+\left\|y_{n}^{k}\right\|_{W^{\alpha, p}\left(J_{k} ; E\right)}^{p}\right] \leq C\left|x_{n}\right|^{p}+C \mathbb{E}\left\|A^{\rho-1} F\left(., \hat{u}_{n}^{k}\right)\right\|_{L^{p}\left(J_{k} ; E\right)}^{p} \\
& \leq C .
\end{aligned}
$$

With the same argument as above we check that (8.3)-(8.5) are correct and uniform w.r.t $k$ on each $J_{k}$ with $k=0, \ldots, 2^{n}-1$. With this fact and the identity $u_{n}=u_{n}^{2^{n}-1}$, we conclude the proof of our proposition.

We will also need the following result.

Proposition 8.2 Suppose that $\left(x_{n}\right)_{n \in \mathbb{N}} \subset E$ is a sequence such that

$$
\left|A^{\rho-\frac{1}{p}}\left[x_{n}-u_{0}\right]\right| \rightarrow 0 \text { as } n \rightarrow \infty .
$$

Then, the sequence $\left(g_{n}\right)_{n \in \mathbb{N}}$ defined by Eq. 8.6 is convergent (and hence the set $\left\{g_{n}: n \in \mathbb{N}\right\}$ is pre-compact) in the Banach space $C\left([0, T] ; D\left(A^{\rho-\frac{1}{p}}\right)\right) \cap L^{p}(0, T ; E)$.

Proof of Proposition 8.2 The convergence in $C\left([0, T] ; D\left(A^{\rho-\frac{1}{p}}\right)\right)$ is obvious. From [28, Theorem 2] we infer that for any $\theta \in\left[\frac{1}{p}-\rho, \frac{1}{p}\right]$ there exists some $C>0$ such that

$$
\sup _{n \in \mathbb{N}}\left\|A^{\theta}\left[g_{n}-e^{-t A} u_{0}\right]\right\|_{L^{p}(0, T ; E)}^{p} \leq C\left|A^{\rho-\frac{1}{p}}\left[x_{n}-u_{0}\right]\right|^{p},
$$

from which we derive the convergence in $L^{p}(0, T ; E)$.

After these preliminary claims we are now ready for the proof of Theorem 3.4 which will be divided into several steps. But before we go further let us define a sequence of Poisson random measures $\left\{\eta_{n}\right\}_{n \in \mathbb{N}}$ by putting $\eta_{n}=\eta$ for all $n \in \mathbb{N}$.

Step (I) The family of the laws of $\left(\left(u_{n}, \eta_{n}\right)\right)_{n \in \mathbb{N}}$ is tight on $\left[L^{p}(0, T ; E) \cap\right.$ $\left.\mathbb{D}\left([0, T] ; D\left(A^{\rho^{\prime}-1}\right)\right)\right] \times M_{I}(Z \times[0, T])$, for any $\rho^{\prime} \in(0, \rho)$.

Proof To simplify notation we set $\mathbf{B}_{0}=D\left(A^{\rho^{\prime}-1}\right)$ for any $\rho^{\prime} \in(0, \rho)$. Define three functions $f_{n}, g_{n}$ and $v_{n}$ by

$$
\begin{gathered}
f_{n}(t)=F\left(t, \hat{u}_{n}(t)\right), \quad t \in[0, T], \\
g_{n}(t ; z)=G\left(s, \hat{u}_{n}(t) ; z\right), \quad t \in[0, T], \quad z \in Z,
\end{gathered}
$$

and

$$
v_{n}(t)=\int_{0}^{t} \int_{Z} e^{-(t-s) A} G\left(s, \hat{u}_{n}(s) ; z\right) \tilde{\eta}(d z ; d s) .
$$

We argue exactly as in [10]. We recall that the space $\mathcal{M}^{p}(0, T ; E)$, the operators $\Lambda$ and $\mathcal{A}$ are defined on page 9 and 7, respectively. Since, by estimates (8.4) and Assumption 5, the 
family $\left(A^{\rho-1} f_{n}\right)_{n \in \mathbb{N}}$ is bounded in $\mathcal{M}^{p}(0, T ; E)$ and $\mathcal{A} \Lambda^{-1}$ is bounded on $L^{p}(0, T ; E)$ it follows from [10, Theorem 2.6] and Lemma 2.2 that $\Lambda^{-1} f_{n}=\Lambda^{-\rho}\left(\mathcal{A} \Lambda^{-1}\right)^{1-\rho} \mathcal{A}^{\rho-1} f_{n}$ is tight on $L^{p}(0, T ; E) \cap C([0, T] ; E)$. This fact, the compact embedding $E \subset \mathbf{B}_{0}$ and the continuity of the embedding

$$
C\left([0, T] ; \mathbf{B}_{0}\right) \subset \mathbb{D}\left(0, T ; \mathbf{B}_{0}\right)
$$

imply that $\Lambda^{-1} f_{n}$ is tight on $L^{p}(0, T ; E) \cap \mathbb{D}\left([0, T] ; \mathbf{B}_{0}\right)$. Next by estimates (8.4), Lemma 7.6 and Lemma 7.7, we infer that the laws of the family $\left(v_{n}\right)_{n \in \mathbb{N}}$ are tight on $L^{p}(0, T ; E) \cap$ $\mathbb{D}\left([0, T] ; \mathbf{B}_{0}\right)$. Finally, from Proposition 8.2 it follows that the family of functions $\left\{e^{-\cdot A} x_{n}\right.$ : $n \in \mathbb{N}\}$ is precompact in $L^{p}(0, T ; E) \cap \mathbb{D}\left([0, T] ; \mathbf{B}_{0}\right)$. Since

$$
u_{n}=v_{n}+\Lambda^{-1} f_{n}+e^{-\cdot A} x_{n}, \quad n \in \mathbb{N},
$$

we easily conclude that the laws of the family $\left(u_{n}\right)_{n \in \mathbb{N}}$ are tight on $L^{p}(0, T ; E) \cap$ $\mathbb{D}\left([0, T] ; \mathbf{B}_{0}\right)$. Since $M_{I}(Z \times[0, T])$ is a separable metric space, by [62, Theorem 3.2] the family of the laws of $\left(\eta_{n}\right)_{n \in \mathbb{N}}$ are tight on $M_{I}(Z \times[0, T])$. Consequently, the family of the laws of $\left(\left(u_{n}, \eta_{n}\right)\right)_{n \in \mathbb{N}}$ is tight on $\mathfrak{Z}_{T}$, where

$$
\mathfrak{Z}_{T}=\left[L^{p}(0, T ; E) \cap \mathbb{D}\left([0, T] ; \mathbf{B}_{0}\right)\right] \times M_{I}(Z \times[0, T]) .
$$

Remark Let us observe that the space $\mathfrak{Z}_{T}$ defined above in Eq. 8.11 differs from the space $\mathfrak{X}_{T}$ defined earlier in Eq. 3.22. From Step (I) and Prokhorov Theorem (see, for instance, [24, Theorem 2.3]) we deduce that there exist a subsequence of $\left(\left(u_{n}, \eta_{n}\right)\right)_{n \in \mathbb{N}}$, still denoted by $\left(\left(u_{n}, \eta_{n}\right)\right)_{n \in \mathbb{N}}$, and a Borel probability measure $\mu_{*}$ on $\mathfrak{Z}_{T}$ such that $\mathcal{L}\left(u_{n}, \eta_{n}\right) \rightarrow \mu_{*}$ weakly. By Theorem C. 1 there exist a probability space $(\bar{\Omega}, \overline{\mathcal{F}}, \overline{\mathbb{P}})$ and a sequence $\left(\bar{u}_{n}, \bar{\eta}_{n}\right)_{n \in \mathbb{N}}$, of $\mathfrak{Z}_{T}$-valued random variables such that

$$
\text { the laws of }\left(\bar{u}_{n}, \bar{\eta}_{n}\right) \text { and }\left(u_{n}, \eta_{n}\right) \text { on } \mathfrak{Z}_{T} \text { are equal, }
$$

and there exists a $\mathfrak{Z}_{T}$-valued random variable $\left(u_{*}, \eta_{*}\right)$ on $(\bar{\Omega}, \overline{\mathcal{F}}, \overline{\mathbb{P}})$ with

$$
\mathcal{L}\left(\left(u_{*}, \eta_{*}\right)\right)=\mu_{*},
$$

such that $\overline{\mathbb{P}}$-a.s.

$$
\left(\bar{u}_{n}, \bar{\eta}_{n}\right) \rightarrow\left(u_{*}, \eta_{*}\right) \text { in } \mathfrak{Z}_{T},
$$

and $\bar{\eta}_{n}=\eta_{*}$ for all $n \in \mathbb{N}$. The sequence $\left(\bar{u}_{n}\right)_{n \in \mathbb{N}}$ has similar properties as the original sequence $\left(u_{n}\right)_{n \in \mathbb{N}}$. Those we will use are stated in part (i) of the next step.

Step (II) The following holds

(i) $\sup _{n \in \mathbb{N}}\left\|\bar{u}_{n}\right\|_{L^{p}(\bar{\Omega} \times[0, T] ; E)}<\infty$ and

(ii) for any $r \in(1, p)$ we have

$$
\lim _{n \rightarrow \infty} \overline{\mathbb{E}}\left\|\bar{u}_{n}-u_{*}\right\|_{L^{p}(0, T ; E)}^{r}=0 .
$$

Proof Let us begin with an observation that in view of Eq. 8.12, for any $n \in \mathbb{N}$, the laws of $u_{n}$ and $\bar{u}_{n}$ on $L^{p}(0, T ; E)$ are identical. Hence,

$$
\left\|u_{n}\right\|_{L^{p}(\Omega \times[0, T] ; E)}=\left\|\bar{u}_{n}\right\|_{L^{p}(\bar{\Omega} \times[0, T] ; E)},
$$

and part (i) easily follows from estimates (8.3). 
Let us fix $r \in(1, p)$. Since $u_{*}$ is $\mathfrak{Z}_{T}$-valued, it follows from part (i) that the sequence $\left\|\bar{u}_{n}-u_{*}\right\|_{L^{p}(0, T ; E)}^{r}$ is $\overline{\mathbb{P}}$-uniformly integrable. Since by Eqs. 8.13 and 8.11 , $\left\|\bar{u}_{n}-u_{*}\right\|_{L^{p}(0, T ; E)}^{r} \rightarrow 0$ on $\bar{\Omega}$, by applying the Vitali Convergence Theorem we deduce part (ii).

Before we continue we should note that the random variables

$$
\bar{u}_{n}, u_{*}: \bar{\Omega} \rightarrow L^{p}(0, T ; E),
$$

induce two $E$-valued stochastic processes still denoted with the same symbols, see for example [18, Proposition B.4] for a proof for the space $L_{l o c}^{\infty}\left(R_{+} ; L_{l o c}^{2}\left(\mathbb{R}^{d}\right)\right)$. Now let $\overline{\mathbb{F}}=\left(\overline{\mathcal{F}}_{t}\right)_{t \geq 0}$ be the filtration defined by

$$
\overline{\mathcal{F}}_{t}=\sigma\left(\sigma\left(\bar{\eta}_{n}(s),\left\{u_{m}(s), m \in \mathbb{N}\right\}, u_{*}(s) ; 0 \leq s \leq t\right) \cup \mathcal{N}\right), t \in[0, T],
$$

where $\mathcal{N}$ denotes the set of null sets of $\overline{\mathcal{F}}$. Since $\bar{\eta}_{n}=\eta_{*}$, it is easy to show that the filtration obtained by replacing $\bar{\eta}_{n}$ with $\eta_{*}$ in Eq. 8.14 is equal to $\overline{\mathbb{F}}$.

The next two steps imply that the following two $E$-valued integrals over the filtered probability space $(\bar{\Omega}, \overline{\mathcal{F}}, \overline{\mathbb{F}}, \overline{\mathbb{P}})$

$$
\left.\int_{0}^{t} \int_{Z} e^{-(t-s) A} G\left(s, \bar{u}_{n}(s), z\right)\right) \tilde{\bar{\eta}}_{n}(d z, d s), \quad t \geq 0,
$$

and

$$
\left.\int_{0}^{t} \int_{Z} e^{-(t-s) A} G\left(s, u_{*}(s), z\right)\right) \tilde{\eta}_{*}(d z, d s), \quad t \geq 0,
$$

do exist.

\section{Step (III) The following holds}

(i) for every $n \in \mathbb{N}, \bar{\eta}_{n}$ is a time-homogeneous Poisson random measure on $\mathcal{B}(Z) \times$ $\mathcal{B}([0, T])$ over $(\bar{\Omega}, \overline{\mathcal{F}}, \overline{\mathbb{F}}, \overline{\mathbb{P}})$ with intensity measure $v \otimes$ Leb.

(ii) $\quad \eta_{*}$ is a time-homogeneous Poisson random measure on $\mathcal{B}(Z) \times \mathcal{B}([0, T])$ over $(\bar{\Omega}, \overline{\mathcal{F}}, \overline{\mathbb{F}}, \overline{\mathbb{P}})$ with intensity measure $v \otimes$ Leb;

Proof Before embarking on the proof, let us first recall that in view of Theorem C.1 we infer that $\bar{\eta}_{n}(\bar{\omega})=\eta^{*}(\bar{\omega})$ for all $\bar{\omega} \in \bar{\Omega}$ and $n \in \mathbb{N}$.

For a random measure $\mu$ on $S \times[0, T]$ and for any $U \in \mathcal{S}$ let us define an $\overline{\mathbb{N}}$-valued process $\left(N_{\mu}(t, U)\right)_{t \geq 0}$ by $N_{\mu}(t, U):=\mu(U \times(0, t]), \quad t \geq 0$. In addition, we denote by $\left(N_{\mu}(t)\right)_{t \geq 0}$ the measure-valued process defined by

$$
N_{\mu}(t)=\left\{\mathcal{S} \ni U \mapsto N_{\mu}(t, U) \in \overline{\mathbb{N}}\right\}, \quad t \in[0, T] .
$$

Proof of Step (III)-(i) Let $U_{1}, \ldots, U_{k} \in \mathcal{Z}$, be $k$ disjoint sets and $t \geq 0$. Since $\eta_{n}$ is a timehomogeneous Poisson random measure and the random variables $N_{\eta_{n}}\left(t, U_{l}\right), l \in\{1, \ldots, k\}$, are independent, we have

$$
\mathbb{E} e^{i\left(\sum_{l=1}^{k} \theta_{l} N_{\eta_{n}}\left(t, U_{l}\right)\right)}=\prod_{l=1}^{k} \mathbb{E} e^{i \theta_{l} N_{\eta_{n}}\left(t, U_{l}\right)} .
$$


Since $\bar{\eta}_{n}$ and $\eta_{n}$ have equal laws, for any $U \in \mathcal{Z}$ and $t \geq 0$, the characteristic functions of the random variables $N_{\eta_{n}}(t, U)$ and $N_{\bar{\eta}_{n}}(t, U)$ are equal. Therefore, it follows from Eq. 8.15 that

$$
\overline{\mathbb{E}} e^{i\left(\sum_{l=1}^{k} \theta_{l} N_{\eta_{n}}\left(t, U_{l}\right)\right)}=\prod_{l=1}^{k} \overline{\mathbb{E}} e^{i \theta_{l} N_{\bar{\eta}_{n}}\left(t, U_{l}\right)},
$$

from which we easily deduce that $\bar{\eta}_{n}$ satisfies Definition 2.3 (a)-(c). In order to finish the proof of part (i) of Step (III) we only need to show that $\bar{\eta}_{n}$ satisfies Definition 2.3 (d) with the filtration defined in Eq. 8.14. For this purpose let us fix $m \in \mathbb{N}, t_{0} \in[0, T]$ and $r \geq s \geq t_{0}$. It follows from the definition of $\overline{\mathbb{F}}$ that $\bar{\eta}_{n}$ is $\overline{\mathbb{F}}$-adapted and it remains to prove that $\bar{X}_{m}=N_{\bar{\eta}_{n}}(r)-N_{\bar{\eta}_{n}}(s)$ is independent of $\overline{\mathcal{F}}_{t_{0}}$. By Definition 2.3 (b) the random variable $\bar{X}_{m}=N_{\bar{\eta}_{n}}(r)-N_{\bar{\eta}_{n}}(s)$ is independent of $N_{\bar{\eta}_{n}}\left(t_{0}\right)$, so we only need to show that $\bar{X}_{m}$ is independent of $\bar{u}_{m}(\sigma)$ and $u_{*}(\sigma)$ for any $\sigma \leq t_{0}$. In what follows we fix $\sigma \in\left[0, t_{0}\right]$. Since $\mathcal{L}\left(\bar{u}_{m}, \bar{\eta}_{m}\right)=\mathcal{L}\left(u_{m}, \eta_{m}\right)$, it follows that

$$
\mathcal{L}\left(\left.\bar{u}_{m}\right|_{[0, \sigma]}, \bar{X}_{m}\right)=\mathcal{L}\left(\left.u_{m}\right|_{[0, \sigma]}, X_{m}\right),
$$

where $X_{m}=N_{\eta_{m}}(r)-N_{\eta_{m}}(s)$. Recall that $\eta_{m}=\eta_{*}$ and $u_{m}$ is the unique solution to the linear stochastic evolution Eq. 8.1, hence it is adapted to the $\sigma$-algebra generated by $\eta_{m}$. Consequently, $\left.u_{m}\right|_{[0, \sigma]}$ is independent of $X_{m}$ and we infer from this last remark and (8.16) that $\left.\bar{u}_{m}\right|_{[0, \sigma]}$ is independent of $\bar{X}_{m}$. The remaining part of the proof, which consists in showing that $\bar{X}_{m}$ is independent of $\left.u_{*}\right|_{[0, \sigma]}$, is addressed in the next lemma.

Lemma 8.3 Assume that $(\Omega, \mathcal{F}, \mathbb{P})$ is a probability space and $Y$ is a Banach space and that $\left(y_{n}\right)_{n \in \mathbb{N}}$ is a sequence of $Y$-valued random variables over $(\Omega, \mathcal{F}, \mathbb{P})$ such that $y_{n} \rightarrow y_{*}$ weakly, i.e., for all $\phi \in Y^{*}, \mathbb{E} e^{i\left\langle\phi, y_{n}\right\rangle} \rightarrow \mathbb{E} e^{i\left\langle\phi, y_{*}\right\rangle}$. If $z$ is a another $Y$-valued random variable over $(\Omega, \mathcal{F}, \mathbb{P})$ such that $y_{n}$ and $z$ are independent for all $n \geq 1$, then $y_{*}$ and $z$ are also independent.

Proof of Lemma 8.3 The random variables $y_{*}$ and $z$ are independent iff

$$
\mathbb{E} e^{i\left(\theta_{1} z+\theta_{2} y_{*}\right)}=\mathbb{E} e^{i \theta_{1} z} \mathbb{E} e^{i \theta_{2} y_{*}}, \quad \theta_{1}, \theta_{2} \in Y^{*} .
$$

The weak convergence and the independence of $z$ and $y_{n}$ for all $n \in \mathbb{N}$ justify the following chain of equalities.

$$
\mathbb{E} e^{i\left(\theta_{1} z+\theta_{2} y_{*}\right)}=\lim _{n \rightarrow \infty} \mathbb{E} e^{i\left(\theta_{1} z+\theta_{2} y_{n}\right)}=\lim _{n \rightarrow \infty} \mathbb{E} e^{i \theta_{1} z} \mathbb{E} e^{\theta_{2} y_{n}}=\mathbb{E} e^{i \theta_{1} z} \mathbb{E} e^{\theta_{2} y_{*}} .
$$

Since $\left.\bar{u}_{m}\right|_{[0, \sigma]}$ is independent from $\bar{X}_{m}$, Lemma 8.3 implies that $\left.u_{*}\right|_{[0, \sigma]}$ is independent of $\bar{X}_{m}$.

Proof of Step (III)-(ii) We have to show that $\eta_{*}$ is a time-homogeneous Poisson random measure with intensity $v \otimes$ Leb. But this will follow from Step (III)-(i), since $\eta_{*}(\omega)=$ $\bar{\eta}_{m}(\omega)$ for all $\omega \in \Omega$ and $m \in \mathbb{N}$.

Step (IV) The following holds

(i) for every $n \in \mathbb{N}$, the process $\bar{u}_{n}$ is a $\overline{\mathbb{F}}$-progressively measurable;

(ii) the E-valued process $u_{*}$ is $\overline{\mathbb{F}}$-progressively measurable. 
Proof One may suspect that there is a simpler proof by by adaptiveness and left-continuity. However, here the problem is that $u_{n} \in D([0, T] ; Y)$ with $E \subset Y$ densely and continuously. Because $G$ is only defined on $[0, T] \times E$, we want $u$ as an $E$-valued process to be progressively measurable.

As we noted earlier, one can argue as in [18, Proposition B.4] and prove that the random variables $\bar{u}_{n}, u_{*}: \bar{\Omega} \rightarrow L^{p}(0, T ; E)$ induce two $E$-valued stochastic processes still denoted with the same symbols. Here, we have to show that for each $n \in \mathbb{N}, \bar{u}_{n}$ and $u_{*}$ are $\overline{\mathbb{F}}$ progressively measurable. By definition of $\overline{\mathbb{F}}$, for fixed $n \in \mathbb{N}$ the process $\bar{u}_{n}$ is adapted to $\overline{\mathbb{F}}$ by the definition of $\overline{\mathbb{F}}$. Let us fix $r \in(1, p)$. By Step (II) the process $\bar{u}_{n}$ is bounded in $L^{r}\left(\bar{\Omega}_{T} ; E\right)$, hence, there exists a sequence of simple functions $\left(\bar{u}_{n}^{m}\right)_{m \in \mathbb{N}}$ such that $\bar{u}_{n}^{m} \rightarrow \bar{u}_{n}$ as $m \rightarrow \infty$ in $L^{r}\left(\bar{\Omega}_{T} ; E\right)$. In particularly, by using the shifted Haar projections used in [15, Appendix B] we can choose $\left(\overline{u_{n}^{m}}\right)_{m \in \mathbb{N}}$ to be progressively measurable. It follows that $\bar{u}_{n}$ is progressively measurable as a $L^{r}\left(\bar{\Omega}_{T} ; E\right)$-limit of a sequence of progressively processes. Finally, since $\bar{u}_{n} \rightarrow u_{*}$ as $n \rightarrow \infty$ also in $L^{r}\left(\bar{\Omega}_{T} ; E\right)$, it follows that $u_{*}$ is progressively measurable.

Let $\mu$ be a time-homogeneous Poisson random measure over $(\bar{\Omega}, \overline{\mathcal{F}}, \overline{\mathbb{F}}, \overline{\mathbb{P}})$ with intensity measure $v \otimes$ Leb, $v$ be an $E$-valued progressively measurable process, $u_{0} \in D\left(A^{\rho-\frac{1}{p}}\right)$ and $\mathcal{K}$ be a nonlinear map defined by

$$
\begin{aligned}
& \mathcal{K}(x, v, \mu)(t):=e^{-t A} u_{0}+\int_{0}^{t} e^{-(t-s) A} F(s, v(s)) d s \\
& \left.\quad+\int_{0}^{t} \int_{Z} e^{-(t-s) A} G(s, v(s) ; z)\right) \tilde{\mu}(d z, d s), \quad t \in[0, T] .
\end{aligned}
$$

Here, as usual, $\tilde{\mu}$ denotes the compensated Poisson random measure of $\mu$.

Step $(\mathbf{V})$ For all $t \in[0, T]$ and $n \in \mathbb{N}$ we have $\overline{\mathbb{P}}$-almost surely

$$
\bar{u}_{n}(t)-\mathcal{K}\left(x_{n}, \hat{\bar{u}}_{n}, \bar{\eta}_{n}\right)(t)=0,
$$

where $\hat{\bar{u}}_{n}$ is defined by

$$
\hat{\bar{u}}_{n}:= \begin{cases}x_{n}, & \text { if } s \in\left[0,2^{-n}\right), \\ 2^{n} \int_{\phi_{n}(s)-2^{-n}}^{\phi_{n}(s)} \bar{u}_{n}(r) d r, & \text { if } s \geq 2^{-n} .\end{cases}
$$

Proof First, let $\rho^{\prime} \in(0, \rho)$ and

$$
\mathfrak{X}_{T}^{1}=L^{p}(0, T ; E) \cap L^{\infty}\left(\mathbb{R}_{+} ; D\left(A^{\rho^{\prime}-1}\right)\right)
$$

and

$$
\mathfrak{X}_{T}^{2}=M_{I}(Z \times[0, T]) .
$$

Again for simplicity we set $\mathbf{B}_{0}=D\left(A^{\rho^{\prime}-1}\right)$. It is proved in [14] that the map $\mathcal{G}: \mathfrak{X}_{T}^{1} \rightarrow \mathfrak{X}_{T}^{1}$ defined by

$$
\mathcal{G}(u)(s):= \begin{cases}x_{n}, & \text { if } s \in\left[0,2^{-n}\right), \\ 2^{n} \int_{\phi_{n}(s)-2^{-n}}^{\phi_{n}(s)} u(r) d r, & \text { if } s \geq 2^{-n}\end{cases}
$$


is well defined, linear and bounded. Therefore, for any $n \in \mathbb{N}$, the two triplets of random variables $\left(u_{n}, \eta_{n}, \hat{u}_{n}\right)$ and $\left(\bar{u}_{n}, \bar{\eta}_{n}, \hat{\bar{u}}_{n}\right)$, where $\hat{u}_{n}=\mathcal{G}\left(u_{n}\right)$ and $\hat{\bar{u}}_{n}=\mathcal{G}\left(\bar{u}_{n}\right)$, have equal laws on $\mathfrak{X}_{T}^{1} \times \mathfrak{X}_{T}^{2}$. Second, let us define processes $\tilde{z}_{n}$ and $\breve{z}_{n}$ by

$$
\begin{aligned}
& \tilde{z}_{n}(t):=e^{-t A} x_{n}+\int_{0}^{t} e^{-(t-s) A} F\left(s, u_{n}(s)\right) d s \\
& +\int_{0}^{t} \int_{Z} e^{-(t-s) A} G\left(s, u_{n}(s, z)\right) \tilde{\eta}(d z, d s), t \in[0, T], \\
& \breve{z}_{n}(t):=e^{-t A} x_{n}+\int_{0}^{t} e^{-(t-s) A} F\left(s, \hat{u}_{n}(s)\right) d s \\
& \quad+\int_{0}^{t} \int_{Z} e^{-(t-s) A} G\left(s, \hat{u}_{n}(s, z)\right) \tilde{\eta}(d z, d s), t \in[0, T] .
\end{aligned}
$$

Let us also define processes $\tilde{\bar{z}}_{n}$ and $\breve{\bar{z}}_{n}$ by replacing $\left(u_{n}, \eta\right)$ and $\left(\hat{u}_{n}, \eta\right)$ by $\left(\bar{u}_{n}, \bar{\eta}_{n}\right)$ and $\left(\hat{\bar{u}}_{n}, \bar{\eta}_{n}\right)$ in formula (8.19) and (8.20), respectively. Thanks to the continuity of the linear map $\mathcal{G}$ and Assumption 5, it follows from [15, Theorem 1] that the quintuples of random variables $\left(u_{n}, \eta_{n}, \hat{u}_{n}, \tilde{z}_{n}, \breve{z}_{n}\right)$ and $\left(\bar{u}_{n}, \bar{\eta}_{n}, \hat{\bar{u}}_{n}, \tilde{\bar{z}}_{n}, \breve{\bar{z}}_{n}\right)$ have the same law on $\mathfrak{Z}_{T} \times \mathfrak{X}_{T}^{1} \times \mathfrak{X}_{T}^{1} \times$ $\mathfrak{X}_{T}^{1}$. Consequently $\Psi\left(u_{n}, \breve{z}_{n}\right)$ and $\Psi\left(\bar{u}_{n}, \breve{\bar{z}}_{n}\right)$ have equal laws on $\mathbb{R}$, where the continuous functional $\Psi: \mathfrak{X}_{T}^{1} \times \mathfrak{X}_{T}^{1} \rightarrow \mathbb{R}$ is defined by

$$
\Psi(v, w)=\int_{0}^{T}|v(t)-w(t)|_{B_{0}} d t, \text { for } v \in \mathfrak{X}_{T} \text { and } w \in \mathfrak{X}_{T} .
$$

Therefore, for any function $\varphi \in C_{b}\left(\mathbb{R}, \mathbb{R}_{+}\right)$we have

$$
\mathbb{E}_{n}\left[\varphi\left(\Psi\left(\bar{u}_{n}, \breve{z}_{n}\right)\right)\right]=\overline{\mathbb{E}}\left[\varphi\left(\Psi\left(\bar{u}_{n}, \breve{\bar{z}}_{n}\right)\right)\right] .
$$

Now let $\varepsilon>0$ be arbitrary and let $\phi_{\varepsilon} \in C_{b}\left(\mathbb{R}, \mathbb{R}_{+}\right)$be defined by

$$
\phi_{\varepsilon}(y)=\left\{\begin{array}{l}
\frac{y}{\varepsilon}, \text { if } y \in[0, \varepsilon), \\
\mathbf{1}_{[\varepsilon, \infty)}(y), \text { otherwise. }
\end{array}\right.
$$

It is easy to check that

$$
\begin{aligned}
\overline{\mathbb{P}}\left(\Psi\left(\bar{u}_{n}, \breve{\bar{z}}_{n}\right) \geq \varepsilon\right) \leq & \int_{\bar{\Omega}} \mathbf{1}_{[\varepsilon, \infty)}\left(\Psi\left(\bar{u}_{n}, \breve{\bar{z}}_{n}\right)\right) d \overline{\mathbb{P}} \\
& +\int_{\bar{\Omega}} \mathbf{1}_{[0, \varepsilon)}\left(\Psi\left(\bar{u}_{n}, \breve{\bar{z}}_{n}\right)\right) \frac{\Psi\left(\bar{u}_{n}, \breve{\bar{z}}_{n}\right)}{\varepsilon} d \overline{\mathbb{P}} \\
= & \overline{\mathbb{E}} \phi_{\varepsilon}\left(\Psi\left(\overline{\bar{u}}_{n}, \overline{\bar{z}}_{n}\right)\right) .
\end{aligned}
$$

The last inequality altogether with Eq. 8.21 implies that

$$
\overline{\mathbb{P}}\left(\Psi\left(\bar{u}_{n}, \breve{\bar{z}}_{n}\right) \geq \varepsilon\right) \leq \mathbb{E}_{n} \phi_{\varepsilon}\left(\Psi\left(u_{n}, \breve{z}_{n}\right)\right) .
$$

Since for any $t$ and $\mathbb{P}$-almost surely $u_{n}(t)-\breve{z}_{n}(t)=0$ we obtain that $\overline{\mathbb{P}}$ almost surely $\Psi\left(u_{n}, \breve{z}_{n}\right)=0$, which along with Eq. 8.22 yield that for any $\varepsilon>0$

$$
\overline{\mathbb{P}}\left(\Psi\left(\bar{u}_{n}, \breve{\bar{z}}_{n}\right) \geq \varepsilon\right)=0 .
$$

Since $\varepsilon>0$ is arbitrary, we infer from the last equation that $\overline{\mathbb{P}}$-a.s.,

$$
\Psi\left(\bar{u}_{n}, \breve{\bar{z}}_{n}\right)=0 .
$$

This implies that for $\overline{\mathbb{P}}$ almost all $t \in[0, T]$ and almost surely $\bar{u}_{n}(t)=\breve{\bar{z}}_{n}(t)$. Since two càdlàg functions which are equal almost all $t \in[0, T]$ must be equal for all $t \in[0, T]$, we derive that almost surely

for all $t \in[0, T]$.

$$
\bar{u}_{n}=\mathcal{K}\left(x_{n}, \hat{\bar{u}}_{n}, \tilde{\bar{\eta}}_{n}\right) \text {, }
$$


Step (VI) We have

$$
\left\|\mathcal{K}\left(x_{n}, \hat{\overline{u_{n}}}, \bar{\eta}_{n}\right)-\mathcal{K}\left(u_{0}, u_{*}, \eta_{*}\right)\right\|_{L^{p}(\bar{\Omega} \times[0, T] ; E)} \rightarrow 0 \text {, as } n \rightarrow \infty .
$$

Proof First, notice that since $\bar{\eta}_{n}=\eta_{*}$ for any $n \in \mathbb{N}$, the convergence in Step (VI) is equivalent to

$$
\overline{\mathbb{E}}\left\|\mathcal{K}\left(x_{n}, \hat{\overline{u_{n}}}, \eta_{*}\right)-\mathcal{K}\left(u_{0}, u_{*}, \eta_{*}\right)\right\|_{L^{p}(0, T ; E)}^{p} \rightarrow 0 \text { as } n \rightarrow \infty .
$$

Observe that for any any $n$

$$
\begin{aligned}
& \overline{\mathbb{E}}\left\|\mathcal{K}\left(x_{n}, \hat{\bar{u}}_{n}, \eta_{*}\right)-\mathcal{K}\left(u_{0}, u_{*}, \eta_{*}\right)\right\|_{L^{p}(0, T ; E)}^{p} \leq C\left\{\overline{\mathbb{E}} \int_{0}^{T}\left|e^{-t A}\left[x_{n}-u_{0}\right]\right|^{p} d t\right. \\
& +\overline{\mathbb{E}} \int_{0}^{T}\left|\int_{0}^{t} e^{-(t-s) A}\left[F\left(s, \hat{\bar{u}}_{n}(s)\right)-F\left(s, u_{*}(s)\right)\right] d s\right|^{p} d t \\
& +\overline{\mathbb{E}} \int_{0}^{T} \mid \int_{0}^{t} \int_{Z} e^{-(t-s) A} G\left(s, \hat{\bar{u}}_{n}(s) ; z\right) \tilde{\eta}_{*}(d z, d s) \\
& \left.\quad-\left.\int_{0}^{t} \int_{Z} e^{-(t-s) A} G\left(s, u_{*}(s) ; z\right) \tilde{\eta}_{*}(d z, d s)\right|^{p} d t\right\} \\
& =: C\left(S_{0}^{n}+S_{1}^{n}+S_{2}^{n}\right) .
\end{aligned}
$$

Since $A^{\rho-\frac{1}{p}} x_{n} \rightarrow A^{\rho-\frac{1}{p}} u_{0}$ in $E$, the Lebesgue DCT implies

$$
S_{0}^{n} \leq C_{1} \int_{0}^{T}\left\|A^{-\left(\rho-\frac{1}{p}\right)} e^{-t A}\right\|_{\mathcal{L}(E, E)}\left|\left(A^{\rho-\frac{1}{p}} u_{0}-A^{\rho-\frac{1}{p}} x_{n}\right)\right|^{p} d t \longrightarrow 0,
$$

as $n \rightarrow \infty$.

Since $\bar{u}_{n}=\mathcal{K}\left(x_{n}, \hat{\bar{u}}_{n}, \tilde{\eta}_{*}\right)$, arguing as in the proof Proposition 8.1 we can show that $\bar{u}_{n} \in W^{\alpha, p}(0, T, E) \overline{\mathbb{P}}$-a.s., for any $\alpha \in\left(0, \frac{1}{p}\right)$. Hence by inequality (E.1) it follows that $\overline{\mathbb{P}}$-a.s.

$$
\left\|\hat{\bar{u}}_{n}-u_{*}\right\|_{L^{p}(0, T ; E)}^{p} \leq C\left\|\bar{u}_{n}-u_{*}\right\|_{L^{p}(0, T ; E)}^{p}+C 2^{-n p \alpha}\left\|\bar{u}_{n}\right\|_{W^{\alpha, p}(0, T ; E)}^{p} .
$$

Hence, in view of Eq. 8.13 and the continuous embedding $E \subset X$ we infer that

$$
\lim _{n \rightarrow \infty} \hat{\overline{u_{n}}}=u_{*} \text { in } L^{p}(0, T ; X), \quad \overline{\mathbb{P}} \text { a.s. . }
$$

Next, by the Young inequality we infer that

$$
\begin{aligned}
\int_{0}^{T}\left|\int_{0}^{t} e^{-(t-s) A}\left[F\left(s, \hat{\bar{u}}_{n}(s)\right)-F\left(s, u_{*}(s)\right)\right] d s\right|^{p} d t \\
\leq C \int_{0}^{T}\left|A^{\rho-1} F\left(s, \hat{\bar{u}}_{n}(s)\right)-A^{\rho-1} F\left(s, u_{*}(s)\right)\right|^{p} d s,
\end{aligned}
$$

for some $C>0$. By Assumption 5 and since $\bar{u}_{n}, u_{*} \in L^{p}(0, T ; E) \overline{\mathbb{P}}$-a.s., there exists a constant $C>0$ such that for any $n \in \mathbb{N}$

$$
\int_{0}^{T}\left|A^{\rho-1} F\left(s, \hat{\bar{u}}_{n}(s)\right)\right|^{p} d s \leq C,
$$


and

$$
\int_{0}^{T}\left|A^{\rho-1} F\left(s, u_{*}(s)\right)\right|^{p} d s \leq C .
$$

The continuity of $F$ (see Assumption 5), the convergence (8.23) and the Lebesgue DCT imply

$$
S_{1}^{n} \rightarrow 0 \text { as } n \rightarrow \infty \text {. }
$$

In a similar way we will show that $S_{2}^{n} \rightarrow 0$ as $n \rightarrow \infty$. In particular, from Fubini Theorem and Theorem 7.1 as well as (8.13) we infer that

$$
\begin{aligned}
S_{2}^{n} \leq \overline{\mathbb{E}} \int_{0}^{T} \int_{0}^{t} \int_{Z} & \left|e^{-(t-s) A}\left[G\left(s, \hat{\bar{u}}_{n}(s) ; z\right)-G\left(s, u_{*}(s) ; z\right)\right]\right|^{p} v(d z) d s d t \\
\leq & C \overline{\mathbb{E}} \int_{0}^{T} \int_{Z}\left|A^{\rho-\frac{1}{p}} G\left(s, \hat{\bar{u}}_{n}(s) ; z\right)-A^{\rho-\frac{1}{p}} G\left(s, u_{*}(s) ; z\right)\right|^{p} v(d z) d s,
\end{aligned}
$$

where $C=\left(\int_{0}^{T}\left|A^{\frac{1}{p}-\rho} e^{-s A}\right|^{p} d s\right)$. By Assumption 3 there exists a constant $C>0$ such that

$$
\sup _{n} \int_{Z}\left|A^{\rho-\frac{1}{p}} G\left(s, \hat{\bar{u}}_{n}(s) ; z\right)\right|^{p} v(d z) \leq C,
$$

hence by the continuity of $G$, the convergence (8.23) and the Lebesgue DCT

$$
S_{2}^{n} \rightarrow 0 \text { as } n \rightarrow \infty \text {. }
$$

To establish Theorem 3.4 we need to check the following claim.

Step (VII) We have that $\overline{\mathbb{P}}$-a.s. for all $t \in[0, T] u_{*}(t)=\mathcal{K}\left(u_{0}, u_{*}, \eta_{*}\right)(t)$.

Proof Let us fix $r \in(1, p)$. From Steps (II) to (VI) we infer that $\bar{u}_{n} \rightarrow u_{*}$ in $L^{r}\left(\bar{\Omega}_{T} ; E\right)$,

$$
\bar{u}_{n}=\mathcal{K}\left(x_{n}, \hat{\overline{u_{n}}}, \bar{\eta}_{n}\right) \text { in } L^{r}\left(\bar{\Omega}_{T} ; E\right),
$$

and

$$
\mathcal{K}\left(x_{n}, \hat{u_{n}}, \bar{\eta}_{n}\right)-\mathcal{K}\left(u_{0}, u_{*}, \eta_{*}\right) \rightarrow 0 \text { in } L^{r}\left(\bar{\Omega}_{T} ; E\right) .
$$

By the uniqueness of the limit, we infer that $u_{*}=\mathcal{K}\left(u_{0}, u_{*}, \eta_{*}\right)$ in $L^{r}\left(\bar{\Omega}_{T} ; E\right)$, which implies that $\overline{\mathbb{P}}$-a.s. $u_{*}(t)=\mathcal{K}\left(u_{0}, u_{*}, \eta_{*}\right)(t)$ a.e. $t \in[0, T]$. By equality (8.13) we infer that $\overline{\mathbb{P}}-$ a.s., $u_{*} \in \mathbb{D}\left([0, T] ; \mathbf{B}_{0}\right)$. Hence by combination of Lemmata 7.2 and 7.3 , and $\left[28\right.$, Theorem 2.8] we deduce that $\overline{\mathbb{P}}-$ a.s., $\mathcal{K}\left(u_{0}, u_{*}, \eta_{*}\right)(\cdot) \in \mathbb{D}\left([0, T] ; \mathbf{B}_{0}\right)$. Hence $\overline{\mathbb{P}}$-a.s. $u_{*}(t)=\mathcal{K}\left(u_{0}, u_{*}, \eta_{*}\right)(t)$ for all $t \in[0, T]$.

It follows now from $\operatorname{Step(III)(ii),~Step(IV)(ii)~and~Step(VII)~that~the~system~}$ $\left(\bar{\Omega}, \overline{\mathcal{F}}, \overline{\mathbb{F}}, \overline{\mathbb{P}}, \eta_{*}, u_{*}\right)$ is an $E$-valued martingale solution, satisfying (3.12), to problem (3.1). The process $u_{*}$ has càdlàg paths in $\mathbf{B}_{0}:=D\left(A^{\rho^{\prime}-1}\right)$ for any $\rho^{\prime} \in(0, \rho)$. Since, by assumption, the maps $F$ and $G$ are bounded, the operator $-A$ is the infinitesimal generator of a contraction type semigroup in $D\left(A^{\rho-\frac{1}{p}}\right)$, we easily infer from Lemma 7.3-(iii) along with Eq. 3.12 that the paths of $u_{*}$ are càdlàg in $D\left(A^{\rho-\frac{1}{p}}\right)$. This completes the proof of Theorem 3.4. 


\section{Proof of Theorem 3.2}

In this section we replace the boundedness assumption on $F$ by the dissipativity of the drift $-A+F$. The spaces $E, X$ are as in Assumption 1, and we recall that $E \subset X \subset D\left(A^{\rho-1}\right)$. Before we proceed let us state the following important consequence of Assumption 4.

Lemma 9.1 (See Da Prato [23]) Assume that $X$ is a Banach space, $-A$ a generator of a $C_{0}$ semigroup of bounded linear operators on $X$ and a mapping $F:[0, T] \times X \rightarrow X$ satisfies Assumption 4. Assume that for $\tau \in[0, \infty]$ two continuous functions $z, v:[0, \tau) \rightarrow X$ satisfy

$$
z(t)=\int_{0}^{t} e^{-(t-s) A} F(s, z(s)+v(s)) d s, t<\tau .
$$

Then

$$
|z(t)|_{X} \leq \int_{0}^{t} e^{-k(t-s)} a\left(|v(s)|_{X}\right) d s, \quad 0 \leq t<\tau .
$$

Before giving the proof of Theorem 3.2 let us notice that Assumption 4 implies that

$$
|F(t, y)|_{X} \leq a\left(|y|_{X}\right), \quad t \geq 0, y \in X .
$$

Proof of Theorem 3.2 Without loss of generality we assume that $k=0$. Let $\left(F_{n}\right)_{n \in \mathbb{N}}$ be a sequence of functions from $[0, \infty) \times X$ to $X$ given by Assumption 4(iii). In particular, there exists a sequence $\left(R_{F}^{n}\right)_{n \in \mathbb{N}}$ of positive numbers, such that $\left|F_{n}(s, y)\right|_{X} \leq R_{F}^{n}$ for all $(s, x) \in$ $[0, T] \times X, n \in \mathbb{N}$, and $\left|F_{n}(s, x)-F(s, x)\right|_{X} \rightarrow 0$ as $n \rightarrow \infty$ for all $(s, x) \in[0, T] \times X$. Finally, by the continuity of the embeddings $X \hookrightarrow D\left(A^{\rho-\frac{1}{p}}\right) \subset D\left(A^{\rho-1}\right)$ the family of functions $\left(F_{n}\right)_{n \in \mathbb{N}}, F$ and $G$ satisfy all the assumptions of Theorem 3.4. Hence, we infer from the applicability of Theorem 3.4 that there exists an $E$-valued martingale solution to the following problem

$$
\left\{\begin{array}{l}
d u_{n}(t)=\left[-A u_{n}(t)+F_{n}\left(s, u_{n}(t)\right)\right] d t+\int_{Z} G\left(s, u_{n}(t) ; z\right) \tilde{\eta}_{n}(d z, d t), \\
u_{n}(0)=u_{0}
\end{array}\right.
$$

Let us denote this martingale solution by

$$
\left(\Omega_{n}, \mathcal{F}_{n}, \mathbb{P}_{n}, \mathbb{F}_{n}, \eta_{n}, n\right) .
$$

We denote by $\mathbb{E}_{n}$ the mathematical expectation on $\left(\Omega_{n}, \mathcal{F}_{n}, \mathbb{P}_{n}\right)$.

In view of Theorem 3.4, for each $n \in \mathbb{N}, u_{n}$ has càdlàg paths in $D\left(A^{\rho-\frac{1}{p}}\right)$. Moreover,

$$
u_{n}(t)=e^{-t A} u_{0}+z_{n}(t)+v_{n}(t), t \in[0, T],
$$

where

$$
\begin{aligned}
& v_{n}(t)=\int_{0}^{t} \int_{Z} e^{-(t-s) A} G\left(s, u_{n}(s) ; z\right) \tilde{\eta}_{n}(d z ; d s), \\
& \left.z_{n}(t)=\int_{0}^{t} e^{-(t-s) A} F_{n}\left(s, u_{n}(s)\right)\right) d s .
\end{aligned}
$$

Notice, that $z_{n}(t)=u_{n}(t)-v_{n}(t)-e^{-t A} u_{0}, t \in[0, T]$. Similarly to the proof of Theorem 3.4 the proof of Theorem 3.2 will be divided into several steps. The first two steps are the following. 
Step (I) Let $q_{\max }$ be defined by Eq. 3.7. Then for any $\tilde{q} \in\left(q, q_{\max }\right)$ and $r \in(1, p)$, we have

$$
\sup _{n \in \mathbb{N}} \mathbb{E}_{n}\left\|v_{n}\right\|_{L^{\tilde{q}}(0, T ; E)}^{r}<\infty .
$$

Proof Step (I) follows from Lemma 7.9 and Lemma 10.

Step (II) For any $\tilde{q} \in\left(q, q_{\max }\right)$ and $r \in(1, p)$ defined in Step(I), we have

$$
\sup _{n \in \mathbb{N}} \mathbb{E}_{n} \sup _{0 \leq t \leq T}\left|z_{n}(t)\right|_{X}^{\stackrel{r}{\tilde{q}}}<\infty .
$$

Moreover, the laws of the family $\left(z_{n}\right)_{n \in \mathbb{N}}$ are tight on $C([0, T] ; X)$.

Proof By Lemma 9.1 we infer that for any $T \geq 0$

$$
\begin{aligned}
\sup _{0 \leq t \leq T}\left|z_{n}(t)\right|_{X} \leq & \int_{0}^{T} e^{-k(t-s)} a\left(\left|v_{n}(s)\right|_{X}+\left|e^{-s A} x\right|_{X}\right) d s \\
& \leq C \int_{0}^{T}\left(1+\left|v_{n}(s)\right|_{X}^{q}+\left|e^{-s A} x\right|_{X}^{q}\right) d s .
\end{aligned}
$$

Since the embedding $E \subset X$ is continuous, it follows from Step (I) that there exists $\tilde{q}>q$ such that for any $r \in(1, p)$

$$
\sup _{n} \mathbb{E}_{n}\left|v_{n}\right|_{L^{\tilde{q}}(0, T ; X)}^{r}<\infty
$$

Therefore,

$$
\sup _{n} \mathbb{E}_{n} \sup _{0 \leq t \leq T}\left|z_{n}(t)\right|_{X}^{\frac{r}{\tilde{q}}}<\infty .
$$

Hence, we proved the first part of Step (II). Note that the last inequality implies that $\sup _{n} \mathbb{E}_{n}\left|z_{n}\right|_{L^{\tilde{q}}(0, T ; X)}^{\stackrel{r}{\tilde{q}}}<\infty$.

Before we proceed further, we recall that there exist $\theta<1-\frac{q}{q_{\max }}$ and an UMD, type $p$ and separable Banach space $Y$ such that $D\left(A_{Y}^{\theta}\right) \subset X \subset Y$. To prove the second part we use the identity $z_{n}=\Lambda_{Y}^{-1} F_{n}\left(s, u_{n}(s)\right)$, where $\Lambda_{Y}^{-1}=B+\mathcal{A}_{Y}$ with $\mathcal{A}_{Y}$ being defined as in Eq. 2.6 by replacing $A$ with $A_{Y}$, and Remark 2.1 along with Lemma 2.2. But first we need to show that for some $\tilde{p} \in\left(1, \frac{q_{\max }}{q}\right)$, the sequence $\left(\left|F_{n}\left(\cdot, u_{n}(\cdot)\right)\right|_{L^{\tilde{p}}(0, T ; Y)}\right)_{n \in \mathbb{N}}$ is bounded in probability. Let us fix $\tilde{p} \in\left(1, \frac{q_{\max }}{q}\right)$. By Lemma 9.1 and the continuity of the embedding $E \subset X$ we infer that

$$
\left|F_{n}\left(s, u_{n}(s)\right)\right|_{X}^{\tilde{p}} \leq C\left(1+\left|e^{-s A} u_{0}\right|_{X}^{\tilde{p}}+\left|v_{n}(s)\right|_{X}^{\tilde{p} q}+\left|z_{n}(s)\right|_{X}^{\tilde{p} q}\right) .
$$

From this inequality we easily deduce that

$$
\left|F_{n}\left(\cdot, u_{n}(\cdot)\right)\right|_{L^{\tilde{p}}(0, T ; X)}^{\tilde{p}} \leq C\left(1+\left|v_{n}\right|_{L^{\tilde{q}}(0, T ; X)}^{\tilde{p} q}+\left|z_{n}\right|_{L^{\infty}(0, T ; X)}^{\tilde{p} q}\right) .
$$

Taking $\tilde{q}=\tilde{p} q \in\left(q, q_{\max }\right)$ and raising to the power $\frac{r}{\tilde{q}^{2}}$ both sides of Eq. 9.10 implies that

$$
\left|F_{n}\left(\cdot, u_{n}(\cdot)\right)\right|_{L^{\tilde{p}}(0, T ; X)}^{\frac{r \tilde{p}}{\tilde{q}^{2}}} \leq C\left(1+\left|v_{n}\right|_{L^{\tilde{q}}(0, T ; X)}^{\frac{r}{\tilde{q}}}+\left|z_{n}\right|_{L^{\infty}(0, T ; X)}^{\frac{r}{\tilde{\tilde{q}}}} .\right.
$$


Since $Y \subset X$ is continuous we obtain that

$$
\left|F_{n}\left(\cdot, u_{n}(\cdot)\right)\right|_{L^{\tilde{p}}(0, T ; Y)}^{\frac{r \tilde{p}}{\tilde{q}^{2}}} \leq C\left(1++\left|v_{n}\right|_{L^{\tilde{q}}(0, T ; X)}^{\frac{r}{\tilde{q}}}+\left|z_{n}\right|_{L^{\infty}(0, T ; X)}^{\frac{r}{\tilde{q}}}\right) .
$$

By Chebyshev inequality we derive that for any $m \in \mathbb{N}$

$$
\begin{aligned}
& \mathbb{P}_{n}\left(\left|F_{n}\left(s, u_{n}(s)\right)\right|_{L^{\tilde{p}}(0, T ; Y)} \geq m\right) \leq \frac{1}{m^{\frac{r}{q \tilde{q}}}}\left(C+\mathbb{E}_{n}\left|v_{n}(s)\right|_{L^{\tilde{q}}(0, T ; X)}^{\frac{r}{\tilde{q}}}\right. \\
& \left.+\mathbb{E}_{n}\left|z_{n}(s)\right|_{L^{\tilde{q}}(0, T ; X)}^{\frac{r}{\tilde{q}}}\right) .
\end{aligned}
$$

Since $\mathbb{E}_{n}\left|v_{n}(s)\right|_{L^{\tilde{q}}(0, T ; X)}^{\stackrel{r}{\tilde{\tilde{q}}}}$ and $\mathbb{E}_{n}\left|z_{n}(s)\right|_{L^{\tilde{q}}(0, T ; X)}^{\stackrel{r}{\tilde{\tilde{q}}}}$ are uniformly bounded w.r.t $n$, we derive that $\sup _{n \in \mathbb{N}}\left|F_{n}\left(\cdot, u_{n}(\cdot)\right)\right|_{L^{\tilde{p}}(0, T ; Y)}$ is bounded in probability for any $\tilde{p} \in\left(1, \frac{q_{\max }}{q}\right)$.

Next we choose $\tilde{p} \in\left(1, \frac{q_{\max }}{q}\right)$ such that $\theta \in\left(0,1-\frac{1}{\tilde{p}}\right)$. Since $\theta<1-\frac{1}{\tilde{p}}$ and $D\left(A_{Y}^{\theta}\right) \subset X$, we can infer from Lemma 2.2 that the family of the laws of $\left(z_{n}=\Lambda^{-1} F\left(\cdot, u_{n}(\cdot)\right)\right)_{n \in \mathbb{N}}$ is tight on $C\left([0, T] ; D\left(A_{Y}^{\theta}\right)\right)$ and hence on $C([0, T] ; X)$.

Remark 9.2 Let $q$ be a number in the interval [ $p, \infty)$. It follows from Step (I) and Step (II) that for any $\tilde{q} \in\left(q, q_{\max }\right)$ and $r \in(1, p)$

$$
\sup _{n \geq 1} \mathbb{E}_{n}\left\|u_{n}\right\|_{L^{\tilde{q}}(0, T ; X)}^{\frac{r}{\tilde{\tilde{q}}}} \leq C .
$$

For $q<p$ the above inequality holds with $\tilde{q}=p$.

Remark 9.3 In [10], the first named author and Gạtarek constructed an approximation of $F$ as follows. Let $\left(F_{n}\right)_{n \in \mathbb{N}}$ be defined by

$$
F_{n}(s, x)=\left\{\begin{array}{l}
F(s, x) \text { if }|x|_{X} \leq n, \\
F\left(s, \frac{n}{|x|_{X}} x\right) \text { otherwise. }
\end{array}\right.
$$

By Eq. 9.2 $\left|F_{n}(s, y)\right| \leq a(n)$, for all $s \geq 0, y \in E$. They solved the Problem (9.3) driven by Wiener noise on the random interval $\left[0, \tau_{n} \wedge T\right]$ where the sequence of stopping times $\left\{\tau_{n}: n \geq 1\right\}$ is defined by

$$
\tau_{n}=\inf \left\{t \in[0, T]:\left|u_{n}(t)\right|_{X} \geq n\right\} .
$$

By proving that $\sup _{t \in[0, T]}\left|u_{n}(t)\right|_{X}$ is uniformly bounded, which implies that $\tau_{n} \uparrow T$ almost surely as $n \rightarrow \infty$, and then using (9.2) and Lemma 2.2 they could show that the laws of $z_{n}$ is tight on $C([0, T] ; X)$. In our framework we know a priori that $u_{n}$ is only càdlàg in $D\left(A^{\rho^{\prime}-1}\right)$ for $\rho^{\prime} \in(0, \rho)$, hence $\tau_{n}$ will not be a well defined stopping time and we will not be able to show that $\sup _{t \in[0, T]}\left|u_{n}(t)\right|_{X}$ is uniformly bounded.

In order to use Theorem C.1 we also need the following.

Step (III) For $\rho^{\prime} \in(0, \rho)$ let $\mathbf{B}_{0}=D\left(A^{\rho^{\prime}-1}\right)$. The family of laws of $\left(v_{n}\right)_{n \in \mathbb{N}}$ is tight on $L^{\tilde{q}}(0, T, E) \cap \mathbb{D}\left([0, T] ; \mathbf{B}_{0}\right)$ and that of $\left(\eta_{n}\right)_{n \in \mathbb{N}}$ is tight on $M_{I}(Z \times[0, T])$.

Proof By Lemma 7.6 and Lemma 7.7 the laws of the family $\left(v_{n}\right)_{n \in \mathbb{N}}$ are tight on $L^{\tilde{q}}(0, T, E) \cap \mathbb{D}\left([0, T], \mathbf{B}_{0}\right)$. Since $\mathbb{E}_{n} \eta_{n}(A, I)=\mathbb{E} \eta(A, I)$ for all $A \in \mathcal{B}(Z)$ and $I \in \mathcal{B}([0, T])$, the laws of $\eta_{n}$ and $\eta_{m}$ on $M_{I}(Z \times[0, T])$ are identical for any $n, m \in \mathbb{N}$. 
Hence, we can deduce from [62, Theorem 3.2] that the laws of the family $\left(\eta_{n}\right)_{n \in \mathbb{N}}$ are tight on $M_{I}(Z \times[0, T])$.

Let $\mathbf{B}_{0}$ as in Step (III). From Steps (I), (II), (III) and Prokhorov's theorem it follows that there exists a subsequence of $\left(\left(z_{n}, v_{n}, \eta_{n}\right)\right)_{n \in \mathbb{N}}$, also denoted by $\left(\left(z_{n}, v_{n}, \eta_{n}\right)\right)_{n \in \mathbb{N}}$ and a Borel probability measure $\mu_{*}$ on $\mathfrak{X}_{T}$, where the space has been defined earlier in Eq. 3.22, such that the sequence of laws of $\left(z_{n}, v_{n}, \eta_{n}\right)_{n \in \mathbb{N}}$ converges to $\mu_{*}$. Moreover, by Theorem C.1, there exists a probability space $(\hat{\Omega}, \hat{\mathcal{F}}, \hat{\mathbb{P}})$ and $\mathfrak{X}_{T}$-valued random variables $\left(z_{*}, v_{*}, \eta_{*}\right)$, $\left(\hat{z}_{n}, \hat{v}_{n}, \hat{\eta}_{n}\right), n \in \mathbb{N}$, such that $\hat{\mathbb{P}}_{-}$a.s.,

$$
\left(\hat{z}_{n}, \hat{v}_{n}, \hat{\eta}_{n}\right) \rightarrow\left(z_{*}, v_{*}, \eta_{*}\right) \text { in } \mathfrak{X}_{T}
$$

and, for all $n \in \mathbb{N}, \hat{\eta}_{n}=\eta_{*}$ and

$$
\mathcal{L}\left(\left(\hat{z}_{n}, \hat{v}_{n}, \hat{\eta}_{n}\right)\right)=\mathcal{L}\left(\left(z_{n}, v_{n}, \eta_{n}\right)\right) \text { on } \mathfrak{X}_{T} .
$$

We define a filtration $\hat{\mathbb{F}}=\left(\hat{\mathcal{F}}_{t}\right)_{t \in[0, T]}$ on $(\hat{\Omega}, \hat{\mathcal{F}})$ as the one generated by $\eta_{*}, z_{*}, v_{*}$ and the families $\left\{z_{n}: n \in \mathbb{N}\right\}$ and $\left\{v_{n}: n \in \mathbb{N}\right\}$, that is, for $t \in[0, T]$,

$$
\hat{\mathcal{F}}_{t}=\sigma\left(\sigma\left(z_{n}(s),\left\{v_{n}(s), n \in \mathbb{N}\right\}, z_{*}(s), v_{*}(s), \eta_{*}(s) ; 0 \leq s \leq t\right) \cup \mathcal{N}\right),
$$

where $\mathcal{N}$ denotes the set of null sets of $\hat{\mathcal{F}}$.

The next two steps imply that the following two Itô integrals over the filtered probability $\operatorname{space}(\hat{\Omega}, \hat{\mathcal{F}}, \hat{\mathbb{F}}, \hat{\mathbb{P}})$

$$
\left.\int_{0}^{t} \int_{Z} e^{-(t-s) A} G\left(s, \hat{v}_{n}(s)+\hat{z}_{n}(s), z\right)\right) \tilde{\hat{\eta}}_{n}(d z, d s), \quad t \in[0, T],
$$

and

$$
\left.\int_{0}^{t} \int_{Z} e^{-(t-s) A} G\left(s, v_{*}(s)+z_{*}(s), z\right)\right) \tilde{\eta}_{*}(d z, d s), \quad t \in[0, T],
$$

are well defined.

Step (IV) The following hold

(i) for all $n \in \mathbb{N}$, $\hat{\eta}_{n}$ is a time-homogeneous Poisson random measure on $\mathcal{B}(Z) \times \mathcal{B}([0, T])$ $\operatorname{over}(\hat{\Omega}, \hat{\mathcal{F}}, \hat{\mathbb{F}}, \hat{\mathbb{P}})$ with intensity measure $v \otimes$ Leb.

(ii) $\eta_{*}$ is a time-homogeneous Poisson random measure on $\mathcal{B}(Z) \times \mathcal{B}(0, T)$ over $(\hat{\Omega}, \hat{\mathcal{F}}, \hat{\mathbb{F}}, \hat{\mathbb{P}})$ with intensity measure $v \otimes$ Leb;

Step (V) The following holds

(i) for all $n \in \mathbb{N}$, the processes $\hat{v}_{n}$ and $\hat{z}_{n}$ are $\hat{\mathbb{F}}$-progressively measurable;

(ii) the processes $z_{*}$ and $v_{*}$ are $\hat{\mathbb{F}}$-progressively measurable.

The proofs of Step (IV) and (V) are the same as the proofs of Step (IV) and (V) of Theorem 3.4. Also, as earlier in the proof of Theorem 3.4, in order to complete the proof of Theorem 3.2 we have to prove the following claim.

Step (VI) Let $u_{*}=e^{-\cdot A} u_{0}+z_{*}+v_{*}$ and $\mathcal{K}$ be the mapping defined by

$$
\begin{aligned}
\mathcal{K}\left(u_{0}, u, \eta\right)(t)= & e^{-t A} u_{0}+\int_{0}^{t} e^{-(t-s) A} F(s, u(s)) d s \\
& +\int_{0}^{t} \int_{Z} e^{-(t-s) A} G(s, u(s) ; z) \tilde{\eta}(d z ; d s),
\end{aligned}
$$


for $t \in[0, T], u \in \mathbb{D}([0, T], B)$ and $\eta \in M_{I}(Z \times[0, T])$. Then $\hat{\mathbb{P}}$-a.s.,

$$
u_{*}(t)=\mathcal{K}\left(u_{0}, u_{*}, \eta_{*}\right)(t), \quad \forall t \in[0, T] .
$$

Proof Since the process $u_{n}=e^{-\cdot A} u_{0}+z_{n}+v_{n}$ is a martingale solution of problem (9.3) and

$$
\mathcal{L}\left(\left(\hat{z}_{n}, \hat{v}_{n}, \hat{\eta}_{n}\right)\right)=\mathcal{L}\left(\left(z_{n}, v_{n}, \eta_{n}\right)\right),
$$

for all $n \in \mathbb{N}$, we can argue as in Step $(\mathrm{V})$ of the previous section and prove that $\hat{\mathbb{P}}$-a.s.

$$
\hat{u}_{n}(t)=\mathcal{K}_{n}\left(u_{0}, \hat{u}_{n}, \hat{\eta}_{n}\right)(t),
$$

for all $t \in[0, T]$. Here $\mathcal{K}_{n}$ is obtained by replacing $F$ with $F_{n}$ in the definition of $\mathcal{K}$. Since the laws of $z_{n}$ and $\hat{z}_{n}$ are equal on $C([0, T] ; X)$, by Eqs. 9.8 and 9.7 we infer that

$$
\sup _{n \geq 1} \mathbb{E}_{n}\left\|\hat{z}_{n}\right\|_{C([0, T] ; X)}^{\frac{r}{\tilde{q}}}<\infty
$$

Since $v_{n}$ and $\hat{v}_{n}$ have equal laws on $L^{\tilde{q}}(0, T ; E)$, from Eq. 9.6 we deduce that

$$
\sup _{n \geq 1} \mathbb{E}_{n}\left\|\hat{v}_{n}\right\|_{L^{\tilde{q}}(0, T ; E)}^{r}<\infty .
$$

Invoking (9.12) we infer that, $\hat{\mathbb{P}}$-a.s., as $n \rightarrow \infty$,

$$
\left\|\hat{z}_{n}-z_{*}\right\|_{C([0, T] ; X)}^{\frac{r}{2 \tilde{q}}} \rightarrow 0 \text { and }\left\|\hat{z}_{n}\right\|_{C([0, T] ; X)}^{\frac{r}{2 \tilde{q}}} \rightarrow\left\|z_{*}\right\|_{C([0, T] ; X)}^{\frac{r}{2 \tilde{q}}} \cdot
$$

Thanks to Eq. 9.16 the sequence $\left\|\hat{z}_{n}\right\|_{C([0, T] ; X)}^{\frac{r}{2 \tilde{q}}}$ is $\hat{\mathbb{P}}$-uniformly integrable. Thus, the applicability of the Vitali Convergence Theorem implies that

$$
\lim _{n \rightarrow \infty} \mathbb{E}_{n}\left\|\hat{z}_{n}\right\|_{C([0, T] ; X)}^{\frac{r}{2 \tilde{q}}}=\mathbb{E}_{n}\left\|z_{*}\right\|_{C([0, T] ; X)}^{\frac{r}{2 \tilde{q}}} .
$$

Thanks to Eq. 9.16 and this last convergence we can prove, by a similar argument used as above, that

$$
\lim _{n \rightarrow \infty} \mathbb{E}_{n}\left\|\hat{z}_{n}-z_{*}\right\|_{C([0, T] ; X)}^{\frac{r}{2 \tilde{q}}}=0 .
$$

With a similar argument we can also show that

$$
\lim _{n \rightarrow \infty} \mathbb{E}_{n}\left\|\hat{v}_{n}-v_{*}\right\|_{L^{\tilde{q}}\left(0, T ; \mathbf{B}_{0}\right)}^{\frac{r}{2 \tilde{q}}}=0,
$$

and

$$
\mathbb{E}_{n}\left\|\hat{v}_{n}-v_{*}\right\|_{L^{\tilde{q}}(0, T ; E)}^{\frac{r}{2 \tilde{r}}} \rightarrow 0 .
$$

We derive from Eqs. 9.18, 9.19 and 9.20 that

$$
\lim _{n \rightarrow \infty} \mathbb{E}_{n}\left\|\hat{u}_{n}-u_{*}\right\|_{L^{\tilde{q}}\left(0, T ; \mathbf{B}_{0}\right)}^{\frac{r}{2 \tilde{q}}}=0,
$$

and

$$
\lim _{n \rightarrow \infty} \mathbb{E}_{n}\left\|\hat{u}_{n}-u_{*}\right\|_{L^{\tilde{q}}(0, T ; X)}^{\frac{r}{2 \tilde{q}}}=0,
$$


which is correct because the embedding $E \subset X$ is continuous. In the other hand, by Proposition 2.5 we have

$$
\begin{aligned}
& \mathbb{E}_{n}\left\|\mathcal{K}_{n}\left(x, \hat{u}_{n}, \hat{\eta}_{n}\right)-\mathcal{K}\left(x, u_{*}, \eta_{*}\right)\right\|_{L^{p^{*}}\left(0, T ; \mathbf{B}_{0}\right)}^{\tilde{r}} \\
& =\mathbb{E}_{n}\left\|\mathcal{K}_{n}\left(x, \hat{u}_{n}, \eta_{*}\right)-\mathcal{K}\left(x, u_{*}, \eta_{*}\right)\right\|_{L^{p^{*}}\left(0, T ; \mathbf{B}_{0}\right)}^{\tilde{r}}\left(s, \hat{u}_{*}(s)\right)-F\left(s, u_{*}(s)\right) d s \|_{L^{p^{*}}(0, T ; X)}^{\tilde{r}} \\
& \quad \leq C \mathbb{E}_{n} \| \int_{0}^{t} e^{-(t-s) A}\left(F_{n}\left(s, \hat{u}_{n}\right) \|_{\mathcal{M}^{p}(0, T ; E)}\right. \\
& \quad+C \| \int_{0}^{t} \int_{\mathcal{Z}} e^{-(t-s) A}\left(G\left(s, \hat{u}_{n}(s)\right)-G\left(s, u_{*}(s)\right)\right) \tilde{\eta}_{*}(d z, d s) \\
& \leq \quad I_{1}^{n}+I_{2}^{n},
\end{aligned}
$$

where $\tilde{r}=\frac{r}{2 \tilde{q} q}$ and $p^{*}=\min \left(\frac{\tilde{q}}{q}, p\right)$. Arguing as in Step (VI) of the previous section we can show that $I_{2}^{n} \rightarrow 0$ as $n \rightarrow 0$. To deal with $I_{1}^{n}$ we first use Assumption 4(iii) given on page 16 and (9.12) to derive that $\mathbb{P}_{n}$-a.s.

$$
\left\|F_{n}\left(\cdot, \hat{u}_{n}\right)-F\left(\cdot, u_{*}\right)\right\|_{L^{p^{*}}(0, T ; X)}^{\tilde{r}} \rightarrow 0,
$$

as $n \rightarrow \infty$. Since, by Eqs. 9.10, 9.16 and 9.17,

$$
\sup _{n \geq 1} \mathbb{E}_{n}\left\|F_{n}\left(\cdot, \hat{u}_{n}\right)\right\|_{L^{p^{*}}(0, T ; X)}^{2 \tilde{r}}<\infty,
$$

we can apply the Lebesgue DCT and deduce that $I_{1}^{n} \rightarrow 0$ as $n \rightarrow \infty$. Therefore, as $n \rightarrow \infty$

$$
\begin{aligned}
\mathbb{E}_{n}\left\|\hat{u}_{n}-u_{*}\right\|_{L^{p^{*}}\left(0, T ; \mathbf{B}_{0}\right)}^{\tilde{r}} & \rightarrow 0, \\
\mathbb{E}_{n}\left\|\mathcal{K}_{n}\left(x, \hat{u}_{n}, \hat{\eta}_{n}\right)-\mathcal{K}\left(x, u_{*}, \eta_{*}\right)\right\|_{L^{p^{*}}\left(0, T ; \mathbf{B}_{0}\right)}^{\tilde{r}} & \rightarrow 0,
\end{aligned}
$$

as $n \rightarrow \infty$. These two facts along with Eq. 9.15 implies that $\hat{\mathbb{P}}$-a.s. and for a.e. $t \in[0, T]$

$$
u_{*}(t)=\mathcal{K}\left(x, u_{*}, \eta_{*}\right)(t) .
$$

Since $u_{*}$ and $\mathcal{K}\left(x, u_{*}, \eta_{*}\right)$ are $\mathbf{B}_{0}$-valued càdlàg functions, the last equation holds for all $t \in[0, T]$.

It follows now from $\operatorname{Step}(\mathbf{I V})(\mathbf{i i}), \operatorname{Step}(\mathbf{V})(\mathbf{i i )}$ and $\operatorname{Step}(\mathbf{V I})$ that the system $\left(\hat{\Omega}, \hat{\mathcal{F}}, \hat{\mathbb{F}}, \hat{\mathbb{P}}, \eta_{*}, u_{*}\right)$ is an $X$-valued martingale solution to problem (3.1) with càdlàg paths in $\mathbf{B}_{0}:=D\left(A^{\rho^{\prime}-1}\right)$ for any $\rho^{\prime} \in(0, \rho)$. Since $-A$ is the infinitesimal generator of a contraction type $C_{0}$-semigroup on $D\left(A^{\rho-\frac{1}{p}}\right)$ and $u_{*} \in L^{\tilde{q}}(0, T ; X)$ almost surely, then we easily infer from Lemma 7.3-(iii) that the paths of $u_{*}$ are càdlàg in $D\left(A^{\rho-\frac{1}{p}}\right)$. Similar calculations as done in Steps (I) and (II), see also Remark 9.2, yield that for any $\tilde{q} \in\left(q, q_{\max }\right)$ and $r \in(1, p)$

$$
\mathbb{E}\left\|u_{*}\right\|_{L^{\tilde{q}}(0, T ; X)}^{\frac{r}{\tilde{q}}}<\infty .
$$

This completes the proof of Theorem 3.2.

Acknowledgments Open access funding provided by Montanuniversität Leoben. The research by E. Hausenblas and P. A. Razafimandimby has been funded by the FWF-Austrian Science Fund through the project P21622. The research on this paper was initiated during the visit of Hausenblas to the University of York in October 2008. She would like to thank the Mathematics Department at York for hospitality. A major part of this paper was written when Razafimandimby was an FWF Lise Meitner fellow (with project number M1487) at the Montanuniversität Leoben. He is very grateful to the FWF and the Montanuniversität Leoben for their support. Razafimandimby's current research is partially National supported by the National Research Foundation South Africa (Grant number 109355). The authors would like to thank Jerzy Zabczyk 
for discussion related to the dual predictable projection of a Poisson random measure and to Szymon Peszat for discussion related to an example from his paper [64].

We also would like thank the anonymous referees for their insightful comments and help to clarify issues from previous version of the paper; in particular for their help in clarifying the construction of stochastic integral with respect to Poisson random measure (PRM) and progressively measurable integrands.

Last but not least, the authors would like to thank Carl Chalk, Pani Fernando, Ela Motyl, Markus Riedle, Akash Panda and Nimit Rana for a careful reading of the manuscript. Earlier versions of this paper can be found on arXiv: 1010.5933 .

Open Access This article is distributed under the terms of the Creative Commons Attribution 4.0 International License (http://creativecommons.org/licenses/by/4.0/), which permits unrestricted use, distribution, and reproduction in any medium, provided you give appropriate credit to the original author(s) and the source, provide a link to the Creative Commons license, and indicate if changes were made.

\section{Appendix A: Stochastic Appendices}

\section{A.1 Space-time Poissonian White Noise}

Analogously to the space-time Gaussian white noise one can construct a space-time Lévy white noise or space-time Poissonian white noise. But before doing this, let us recall the definition of a Gaussian white noise, see for e.g. Dalang [22].

Definition A.1 Let $(\Omega, \mathcal{F}, \mathbb{P})$ be a complete probability space and let $(S, \mathcal{S}, \sigma)$ a measure space. A Gaussian white noise on $(S, \mathcal{S}, \sigma)$ is an $\mathcal{F} / \mathcal{M}(S)$ measurable map

$$
W: \Omega \rightarrow M(S)
$$

satisfying

(i) for every $U \in \mathcal{S}$ such that $\sigma(U)<\infty, W(U):=i_{U} \circ W$ is a Gaussian random variable with mean 0 and variance $\sigma(U)$;

(ii) if $U_{1}, U_{2} \in \mathcal{S}$ are disjoint, then the random variables $W\left(U_{1}\right)$ and $W\left(U_{2}\right)$ are independent and $W\left(U_{1} \cup U_{2}\right)=W\left(U_{1}\right)+W\left(U_{2}\right)$.

The space-time Gaussian white noise can be defined as follows. Let $\mathcal{O} \subset \mathbb{R}^{d}$ be a domain. Put $S=\mathcal{O} \times[0, \infty), \mathcal{S}=\mathcal{B}(\mathcal{O}) \otimes \mathcal{B}([0, \infty))$ and let $\sigma$ be the Lebesgue measure on $S$. The space-time Gaussian white noise is an $M(\mathcal{O})$-valued process $\left\{W_{s t}(r): r \geq 0\right\}$ defined by

$$
W_{s t}(r)=\{\mathcal{B}(\mathcal{O}) \ni U \mapsto W(U \times[0, r)) \in \mathbb{R}\}, \quad r \geq 0,
$$

where $W: \Omega \rightarrow M(S)$ is a Gaussian white noise on $(S, \mathcal{S}, \sigma)$. Let $(\Omega, \mathcal{F}, \mathbb{F}, \mathbb{P})$ be a filtered probability space. We say that the Gaussian white noise $W$ on $(S, \mathcal{S}, \sigma)$ is a space-time Gaussian white noise over $(\Omega, \mathcal{F}, \mathbb{F}, \mathbb{P})$ if the process $\left\{W_{s t}(r): r \geq 0\right\}$ is $\mathbb{F}$-adapted.

One can show that the $M(\mathcal{O})$-valued process $\left\{W_{s t}(r): r \geq 0\right\}$ generates, in a unique way, an $L^{2}(\mathcal{O})$-cylindrical Wiener process $\left(\hat{W}_{r}\right)_{r \geq 0}$, see [12, Definition 4.1]. In particular, for any $U \in \mathcal{B}(\mathcal{O})$ such that $\operatorname{Leb}(U)<\infty$, and any $r \geq 0, \hat{W}_{t}\left(1_{U}\right)=W(U \times[0, r))=W_{s t}(r, U)$.

Analogously, we can define a Lévy white noise and a space-time Lévy white noise.

Definition A.2 Let $(\Omega, \mathcal{F}, \mathbb{P})$ be a complete probability space, $(S, \mathcal{S}, \sigma)$ be a measurable space, $\gamma \in \mathbb{R}$ and let $v$ be a Lévy measure on $\mathbb{R}$. Then a Lévy white noise on $(S, \mathcal{S}, \sigma)$ with intensity jump size measure $v$ is an $\mathcal{F} / \mathcal{M}(S)$-measurable mapping

$$
L: \Omega \rightarrow M(S)
$$

satisfying 
(i) for all $U \in \mathcal{S}$ such that $\sigma(U)<\infty, L(U):=i_{U} \circ L$ is an infinitely divisible random variables satisfying, for all $\theta \in \mathbb{R}$,

$$
\begin{aligned}
& \mathbb{E} e^{i \theta L(U)} \\
& =\exp \left(\sigma(U)\left[i \gamma \theta+\int_{\mathbb{R}}\left(1-e^{i \theta x}-i \theta x 1_{[-1,1]}(x)\right) v(d x)\right]\right) ;
\end{aligned}
$$

(ii) if $U_{1}, U_{2} \in \mathcal{S}$ are disjoint, then the random variables $L\left(U_{1}\right)$ and $L\left(U_{2}\right)$ are independent and $L\left(U_{1} \cup U_{2}\right)=L\left(U_{1}\right)+L\left(U_{2}\right)$.

Definition A.3 Let $(\Omega, \mathcal{F}, \mathbb{P})$ be a complete probability space. Suppose that $\mathcal{O} \subset \mathbb{R}^{d}$ is a domain and let $S=\mathcal{O} \times[0, \infty), \mathcal{S}=\mathcal{B}(\mathcal{O}) \times \mathcal{B}([0, \infty))$ and $\sigma$ the Lebesgue measure on $S$. Let $v$ be a Lévy measure on $\mathbb{R}$. If $L: \Omega \rightarrow M(S)$ is a Lévy white noise on $(S, \mathcal{S}, \sigma)$ with intensity jump size measure $v$, then the $M(\mathcal{O})$-valued process $\left\{L_{s t}(r): r \geq 0\right\}$ defined by

$$
L_{s t}(r)=\{\mathcal{B}(\mathcal{O}) \ni U \mapsto L(U \times[0, r)) \in \mathbb{R}\}, \quad r \geq 0,
$$

is called a space-time Lévy noise on $\mathcal{O}$ with jump size Lévy measure $v$. We say that $L$ is a space time Lévy white noise over a filtered probability space $(\Omega, \mathcal{F}, \mathbb{F}, \mathbb{P})$ iff the corresponding measure-valued process $\left\{L_{s t}(r): r \geq 0\right\}$, is $\mathbb{F}$-adapted.

Remark A.4 If $L$ is a space-time Lévy white noise on $(S, \mathcal{S}$, Leb), then the corresponding $M(\mathcal{O})$-valued process $\left\{L_{s t}(t): t \geq 0\right\}$ is a weakly cylindrical process on $L^{2}(\mathcal{O})$, see Definition 3.2 [2].

As in the case of space-time Gaussian white noise we also introduce the following definition.

Definition A.5 Let us assume that $(\Omega, \mathcal{F}, \mathbb{P})$ is a complete probability space and $v$ be a Lévy measure on $\mathbb{R}$.

(a) A Poissonian white noise with intensity jump size measure $v$ on a measurable space $(S, \mathcal{S}, \sigma)$ is a $\mathcal{F} / \mathcal{M}\left(M_{I}(S \times \mathbb{R})\right)$ measurable mapping

$$
\eta: \Omega \rightarrow M_{I}(S \times \mathbb{R})
$$

satisfying

(i) for all $U \in \mathcal{S} \otimes \mathcal{B}(\mathbb{R})$ with $(\sigma \otimes v)(U)<\infty, \eta(U):=i_{U} \circ \eta$ is a Poisson random variable with parameter $($ Leb $\otimes v)(U)$;

(ii) if the sets $U_{1} \in \mathcal{S} \otimes \mathcal{B}(\mathbb{R})$ and $U_{2} \in \mathcal{S} \otimes \mathcal{B}(\mathbb{R})$ are disjoint, then the random variables $\eta\left(U_{1}\right)$ and $\eta\left(U_{2}\right)$ are independent and $\eta\left(U_{1} \cup U_{2}\right)=\eta\left(U_{1}\right)+\eta\left(U_{2}\right)$, almost surely.

(b) Let $\mathcal{O} \subset \mathbb{R}^{d}$ be a domain. Then, the map $\eta$ defined in Eq. A.1 is called a space-time Poissonian white noise on $\mathcal{O}$ with intensity jump size measure $v$ iff $\eta$ is a Poissonian white noise on $(S, \mathcal{S}$, Leb) with intensity jump size measure $v$, where $S=\mathcal{O} \times[0, \infty)$, $\mathcal{S}=\mathcal{B}(\mathcal{O}) \otimes \mathcal{B}([0, \infty))$.

The corresponding measure-valued process $\left\{\Pi_{t}: t \geq 0\right\}$ defined by

$$
\Pi_{t}: \mathcal{B}(\mathcal{O}) \times \mathcal{B}(\mathbb{R}) \ni(U, C) \mapsto \eta(U \times[0, t) \times C) \in \overline{\mathbb{N}}_{0},
$$

is called an (homogeneous) space-time Poissonian white noise process.

(c) A space-time Poissonian white noise $\eta$ on $\mathcal{O}$ is called a (homogeneous) spacetime Poissonian white noise over a filtered probability space $(\Omega, \mathcal{F}, \mathbb{F}, \mathbb{P})$ iff the 
measure-valued process $\left\{\Pi_{t}: t \geq 0\right\}$ defined above is $\mathbb{F}$-adapted. (Compare this definition with [65, Definition 7.2].)

Theorem A.6 Let $(\Omega, \mathcal{F}, \mathbb{P})$ be a complete probability space, v a Lévy measure on $\mathbb{R}$, $\mathcal{O} \subset \mathbb{R}^{d}$ is a bounded set. Let also

$$
\eta: \Omega \rightarrow M_{I}(S \times \mathbb{R})
$$

be a space-time Poissonian white noise on $\mathcal{O}$ with intensity jump size measure $v$, where $S=\mathcal{O} \times[0, \infty), \mathcal{S}=\mathcal{B}(\mathcal{O}) \otimes \mathcal{B}([0, \infty))$ and Leb the Lebesgue measure on $S$. Let $\mathfrak{M}$ be the random measure defined by

$$
\mathfrak{M}: \Omega \rightarrow M_{I}(\mathcal{O} \times \mathbb{R} \times[0, \infty))
$$

with

$$
\mathfrak{M}(\omega)(U \times C \times D)=\eta(\omega)(U \times C \times D), \quad U \in \mathcal{B}(\mathcal{O}), C \in \mathcal{B}(\mathbb{R}), D \in \mathcal{B}([0, \infty)) .
$$

Then, $\mathfrak{M}$ is a time-homogeneous Poisson random measure on $\mathcal{O} \times \mathbb{R}$ with the intensity measure $\mathfrak{m} \otimes$ Leb satisfying

$$
\mathfrak{m}: \mathcal{B}(\mathcal{O} \times \mathbb{R}) \ni C \mapsto \int_{\mathbb{R}} \int_{\mathcal{O}} 1_{C}(\xi, \zeta) v(d \zeta) d \xi
$$

Proof The proof is very similar to the proof of [65, Proposition 7.21], so we omit it.

Remark A.7 The compensator $\gamma$ of a homogeneous space-time Poissonian white noise with jump size intensity $v$ is a measure on $\mathcal{O} \times \mathbb{R} \times[0, \infty)$ defined by

$$
\mathcal{B}(\mathcal{O}) \times \mathcal{B}(\mathbb{R}) \times \mathcal{B}([0, \infty)) \ni(U, C, I) \mapsto \gamma(U \times C \times I)=\operatorname{Leb}(U) v(C) \operatorname{Leb}(I) .
$$

\section{Appendix B: Besov Spaces and Their Properties}

We follow the approach to the Besov spaces in Runst and Sickel [69, p. 8, Def. 2]. We are interested in the continuity of the mapping $G$ described in Section 5. To be precise, we will prove the following result.

Proposition B.1 Let $p, p^{*} \in(1, \infty)$ be two numbers satisfying $\frac{1}{p}+\frac{1}{p^{*}}=1$. Then for every $f \in \mathcal{S}\left(\mathbb{R}^{d}\right)$ and $a \in \mathbb{R}^{d}$ the tempered distribution $f \delta_{a}$ belongs to the Besov space $B_{p, \infty}^{-\frac{d}{p^{*}}}\left(\mathbb{R}^{d}\right)$ and

$$
\int_{\mathbb{R}^{d}}\left|f \delta_{a}\right|^{p} \underset{B_{p, \infty} \frac{d}{p^{*}}\left(\mathbb{R}^{d}\right)}{ } d a=(2 \pi)^{-\frac{d}{2}} 2^{-\frac{d}{p^{*}}}|f|_{L^{p}\left(\mathbb{R}^{d}\right)}^{p} .
$$

In particular, there exists a unique bounded linear map

$$
\Psi: L^{p}\left(\mathbb{R}^{d}\right) \rightarrow L^{p}\left(\mathbb{R}^{d}, B_{p, \infty}^{-\frac{d}{p^{*}}}\left(\mathbb{R}^{d}\right)\right)
$$

such that $[\Psi(f)](a)=f \delta_{a}, f \in \mathcal{S}\left(\mathbb{R}^{d}\right), a \in \mathbb{R}^{d}$. 
In what follows, for any $f \in L^{p}\left(\mathbb{R}^{d}\right)$ we denote by $f \delta_{a}$ the value of $\Lambda(f)$ at $a$. Observe that $\left\langle f \delta_{a}, \phi\right\rangle=f(a) \phi(a), \phi \in \mathcal{S}\left(\mathbb{R}^{d}\right)$ so that $f \delta_{a}=f(a) \delta_{a}$. Hence, in order to prove (B.1) it is sufficient to prove it for $f=1$, i.e.,

$$
\left|\delta_{a}\right|_{\substack{p \\ B_{p, \infty}}}=(2 \pi)^{-\frac{d}{2}} 2^{-\frac{d}{p^{*}}} .
$$

Let us recall the definition of the Besov spaces as given in [69, Definition 2, pp. 7-8]. First we choose a function $\psi \in \mathcal{S}\left(\mathbb{R}^{d}\right)$ such that $0 \leq \psi(x) \leq 1, x \in \mathbb{R}^{d}$ and

$$
\psi(x)=\left\{\begin{aligned}
1, & \text { if }|x| \leq 1 \\
0 & \text { if }|x| \geq \frac{3}{2}
\end{aligned}\right.
$$

Then put

$$
\left\{\begin{array}{l}
\phi_{0}(x)=\psi(x), x \in \mathbb{R}^{d}, \\
\phi_{1}(x)=\psi\left(\frac{x}{2}\right)-\psi(x), x \in \mathbb{R}^{d}, \\
\phi_{j}(x)=\phi_{1}\left(2^{-j+1} x\right), x \in \mathbb{R}^{d}, \quad j=2,3, \ldots
\end{array}\right.
$$

We will use the definition of the Fourier transform $\mathcal{F}=\mathcal{F}^{+1}$ and its inverse $\mathcal{F}^{-1}$ as in $\left[69\right.$, p. 6]. In particular, with $\langle\cdot, \cdot\rangle$ being the scalar product in $\mathbb{R}^{d}$, we put

$$
\left(\mathcal{F}^{ \pm 1} f\right)(\xi):=(2 \pi)^{-d / 2} \int_{\mathbb{R}^{d}} e^{\mp i\langle x, \xi\rangle} f(x) d x, \quad f \in \mathcal{S}\left(\mathbb{R}^{d}\right), \xi \in \mathbb{R}^{d} .
$$

With the choice of $\phi=\left\{\phi_{j}\right\}_{j=0}^{\infty}$ as above and $\mathcal{F}$ and $\mathcal{F}^{-1}$ being the Fourier and the inverse Fourier transformations (acting on the space $\mathcal{S}^{\prime}\left(\mathbb{R}^{d}\right)$ of Schwartz distributions) we have the following definition.

Definition B.2 Let $s \in \mathbb{R}, 0<p \leq \infty$ and $f \in \mathcal{S}^{\prime}\left(\mathbb{R}^{d}\right)$. If $0<q<\infty$ we put

$$
|f|_{B_{p, q}^{s}}=\left(\sum_{j=0}^{\infty} 2^{s j q}\left|\mathcal{F}^{-1}\left[\phi_{j} \mathcal{F} f\right]\right|_{L^{p}}^{q}\right)^{\frac{1}{q}}=\left\|\left(2^{s j}\left|\mathcal{F}^{-1}\left[\phi_{j} \mathcal{F} f\right]\right|_{L^{p}}\right)_{j \in \mathbb{N}}\right\|_{l q} .
$$

If $q=\infty$ we put

$$
|f|_{B_{p, \infty}^{s}}=\sup _{j \in \mathbb{N}} 2^{s j}\left|\mathcal{F}^{-1}\left[\phi_{j} \mathcal{F} f\right]\right|_{L^{p}}=\left\|\left(2^{s j}\left|\mathcal{F}^{-1}\left[\phi_{j} \mathcal{F} f\right]\right|_{L^{p}}\right)_{j \in \mathbb{N}}\right\| l^{\infty} .
$$

We denote by $B_{p, q}^{s}\left(\mathbb{R}^{d}\right)$ the space of all $f \in \mathcal{S}^{\prime}\left(\mathbb{R}^{d}\right)$ for which $|f|_{B_{p, q}^{s}}$ is finite.

Lemma B.3 If $\varphi \in \mathcal{S}\left(\mathbb{R}^{d}\right), \lambda>0$ and $g(x):=\varphi(\lambda x), x \in \mathbb{R}^{d}$, then

$$
\left|\mathcal{F}^{-1} g\right|_{L^{p}\left(\mathbb{R}^{d}\right)}=\lambda^{d\left(\frac{1}{p-1}\right)}\left|\mathcal{F}^{-1} \varphi\right|_{L^{p}\left(\mathbb{R}^{d}\right)} .
$$

Proof The proof follows from simple calculations so it is omitted.

Proof of Proposition B.1 As remarked earlier it is enough to show equality (B.2). Since $\mathcal{F}^{-1}(\varphi u)=(2 \pi)^{-d / 2}\left(\mathcal{F}^{-1} \varphi\right) *\left(\mathcal{F}^{-1} u\right), \varphi \in \mathcal{S}, u \in \mathcal{S}^{\prime}$ we infer that for $j \in \mathbb{N}^{*}$,

$$
\begin{aligned}
\mid \mathcal{F}^{-1}\left[\left.\phi_{j} \mathcal{F}\left(\delta_{a}\right)\right|_{L^{p}\left(\mathbb{R}^{d}\right)}\right. & =(2 \pi)^{-d / 2}\left|\left(\mathcal{F}^{-1} \phi_{j}\right) * \delta_{a}\right|_{L^{p}\left(\mathbb{R}^{d}\right)} \\
& =(2 \pi)^{-d / 2}\left|\mathcal{F}^{-1} \phi_{j}\right|_{L^{p}\left(\mathbb{R}^{d}\right)} \\
& =(2 \pi)^{-d / 2} 2^{d\left(\frac{1}{p-1}\right)} 2^{-j d\left(\frac{1}{p-1}\right)}\left|\mathcal{F}^{-1} \phi_{1}\right|_{L^{p}\left(\mathbb{R}^{d}\right)} .
\end{aligned}
$$


Hence, $\delta_{a}$ belongs to the Besov space $B_{p, \infty}^{d\left(\frac{1}{p-1}\right)}\left(\mathbb{R}^{d}\right)$ as requested and the equality (B.2) follows immediately.

Corollary B.4 Assume that $\mathcal{O}$ is a bounded and open subset of $\mathbb{R}^{d}$ with boundary $\partial \mathcal{O}$ of class $C^{\infty}$. Let $r, q \in(1, \infty)$ with $q \geq r$ then there exists a unique bounded linear map

$$
\Psi: L^{q}(\mathcal{O}) \rightarrow L^{q}\left(\mathcal{O}, B_{r, \infty}^{-\frac{d}{r}}(\mathcal{O})\right)
$$

such that $[\Psi(f)](a)=f \delta_{a}, f \in L^{q}(\mathcal{O}), a \in \mathcal{O}$. In particular, there exists a constant $C$ such that for any $f \in L^{q}(\mathcal{O})$

$$
\int_{\mathcal{O}}\left|f \delta_{a}\right|_{B_{r, \infty}^{-d\left(1-\frac{1}{r}\right)}(\mathcal{O})}^{q} d a \leq C|f|_{L^{q(\mathcal{O})}}^{q} .
$$

Proof It is enough to prove (B.3) for any $f \in C_{0}^{\infty}(\mathcal{O})$ as that set is dense in $L^{q}(\mathcal{O})$. As before, we first need to show the following version of Eq. B.2

$$
\sup _{a \in \mathcal{O}}\left|\delta_{a}\right|_{B_{r, \infty}^{-\frac{d}{r^{*}}}(\mathcal{O})}<C(r, d)
$$

for a constant $C(r, d)>0$ depending only on $r$ and $d$. For that aim let us fix $a \in \mathcal{O}$ and let us recall that according to Definition 4.2.1 from [73], $\left|\delta_{a}\right|_{B_{r, \infty}-\frac{d}{r^{*}}(\mathcal{O})}$ is equal to infimum of $|u|_{B_{r, \infty}^{-\frac{d}{r^{*}}\left(\mathbb{R}^{d}\right)}}$ over all $u \in B_{r, \infty}^{-\frac{d}{r^{*}}}\left(\mathbb{R}^{d}\right)$ such that $u_{\mid \mathcal{O}}=\delta_{a}$. Thus $\left|\delta_{a}\right|_{B_{r, \infty}\left(\frac{d}{r^{*}}(\mathcal{O})\right.} \leq\left|\delta_{a}\right|_{B_{r, \infty}}^{-\frac{d}{r^{*}}\left(\mathbb{R}^{d}\right)}$ and the result follows by applying (B.2).

Second, let $\Psi$ be the linear map defined on $L^{q}(\mathcal{O})$ by $\Psi f=f \delta_{a}$ for $f \in L^{q}(\mathcal{O})$ and $a \in \mathcal{O}$. Since, by the assumption $q \geq r, L^{q}(\mathcal{O}) \subset L^{r}(\mathcal{O})$ it follows from the first part of the proof that

$$
\begin{aligned}
\int_{\mathcal{O}}|\Psi f(a)|^{q}{ }_{B_{r, \infty}^{-\frac{d}{r^{*}}}(\mathcal{O})} d a & \leq \int_{\mathcal{O}}|f(a)|^{q}\left|\delta_{a}\right|_{B_{r, \infty}^{-\frac{d}{r^{*}}}(\mathcal{O})} d a \\
& \leq \sup _{a \in \mathcal{O}}\left|\delta_{a}\right|_{B_{r, \infty}-\frac{d}{r^{*}}(\mathcal{O})} \int_{\mathcal{O}}|f(a)|^{q} d a \\
& \leq C(r, d)^{q}|f|_{L^{q}(\mathcal{O})}^{q} .
\end{aligned}
$$

The last inequality completes the proof of Corollary B.4.

\section{Appendix C: A generalisation of the Skorohod Representation Theorem}

Within the proofs of Theorems 3.2 and 3.4 we are dealing with the limits of pairs of random variables. For us it was important that certain properties of the pairs are preserved by the Skorohod Representation Theorem. Therefore, we had to use the theorem which is a modified version of the celebrated Skorohod Representation Theorem.

Theorem C.1 Let $(\Omega, \mathcal{F}, \mathbb{P})$ be a probability space and $U_{1}, U_{2}$ be two separable metric spaces. Let $\chi_{n}: \Omega \rightarrow U_{1} \times U_{2}, n \in \mathbb{N}$, be a family of random variables, such that the sequence $\left(\mathcal{L} a w\left(\chi_{n}\right)\right)_{n \in \mathbb{N}}$ is weakly convergent on $U_{1} \times U_{2}$. 
For $i=1,2$ let $\pi_{i}: U_{1} \times U_{2}$ be the projection onto $U_{i}$, i.e.,

$$
U_{1} \times U_{2} \ni \chi=\left(\chi^{1}, \chi^{2}\right) \mapsto \pi_{i}(\chi)=\chi^{i} \in U_{i} .
$$

Finally let us assume that there exists a random variable $\rho: \Omega \rightarrow U_{1}$ such that $\mathcal{L}$ aw $\left(\pi_{1} \circ\right.$ $\left.\chi_{n}\right)=\mathcal{L} a w(\rho), \forall n \in \mathbb{N}$.

Then, there exists a probability space $(\bar{\Omega}, \overline{\mathcal{F}}, \overline{\mathbb{P}})$, a family of $U_{1} \times U_{2}$-valued random variables $\left(\bar{\chi}_{n}\right)_{n \in \mathbb{N}}$, on $(\bar{\Omega}, \overline{\mathcal{F}}, \overline{\mathbb{P}})$ and a random variable $\chi_{*}: \bar{\Omega} \rightarrow U_{1} \times U_{2}$ such that

(i) $\operatorname{L} a w\left(\overline{\chi_{n}}\right)=\mathcal{L} a w\left(\chi_{n}\right), \forall n \in \mathbb{N}$;

(ii) $\bar{\chi}_{n} \rightarrow \chi_{*}$ in $U_{1} \times U_{2} \overline{\mathbb{P}}-$ a.s.;

(iii) $\pi_{1} \circ \overline{\chi_{n}}(\bar{\omega})=\pi_{1} \circ \chi_{*}(\bar{\omega})$ for all $\bar{\omega} \in \bar{\Omega}$.

Proof of Theorem C.1. The proof is a modification of the proof of [24, Chapter 2, Theorem 2.4]. For simplicity, let us put $\mathcal{P} M U_{n}:=\mathcal{L} a w\left(\chi_{n}\right), \mathcal{P} M U_{n}^{1}:=\mathcal{L} a w\left(\pi_{1} \circ \chi_{n}\right), n \in \mathbb{N}$, and $\mathcal{P} M U_{\infty}:=\lim _{n \rightarrow \infty} \mathcal{L}\left(\chi_{n}\right)$. We will generate families of partitions of $U_{1}$ and $U_{2}$. To start with let $\left(x_{i}\right)_{i \in \mathbb{N}}$ and $\left(y_{i}\right)_{i \in \mathbb{N}}$ be dense subsets in $U_{1}$ and $U_{2}$, respectively, and let $\left(r_{i}\right)_{i \in \mathbb{N}}$ be a sequence of natural numbers converging to zero. Some additional condition on the sequence will be given below.

Now let $O_{1}^{1}:=B\left(x_{1}, r_{1}\right),{ }^{7} O_{k}^{1}:=B\left(x_{k}, r_{1}\right) \backslash\left(\cup_{i=1}^{k-1} O_{i}^{1}\right)$ for $k \geq 2$. Similarly, $C_{1}^{1}:=$ $B\left(y_{1}, r_{1}\right), C_{k}^{1}:=B\left(y_{k}, r_{1}\right) \backslash\left(\cup_{i=1}^{k-1} C_{i}^{1}\right)$ for $k \geq 2$. Inductively, we put

$$
\begin{aligned}
O_{i_{1}, \cdots, 1}^{k} & :=O_{i_{1}, \cdots, i_{k-1}}^{k-1} \cap B\left(x_{i_{k}}, r_{k}\right), \\
O_{i_{1}, \cdots, i_{k}}^{k} & :=O_{i_{1}, \cdots, i_{k-1}}^{k-1} \cap B\left(x_{i_{k}}, r_{k}\right) \backslash\left(\cup_{j=1}^{i_{k}-1} O_{i_{1}, \cdots, i_{k-1}, j}^{k}\right), \quad k \geq 2,
\end{aligned}
$$

and similarly, where we will replace " $O$ " by " $C$ "

$$
\begin{aligned}
C_{i_{1}, \cdots, 1}^{k} & :=C_{i_{1}, \cdots, i_{k-1}}^{k-1} \cap B\left(y_{i_{k}}, r_{k}\right), \\
C_{i_{1}, \cdots, i_{k}}^{k} & :=C_{i_{1}, \cdots, i_{k-1}}^{k-1} \cap B\left(y_{i_{k}}, r_{k}\right) \backslash\left(\cup_{j=1}^{i_{k}-1} C_{i_{1}, \cdots, i_{k-1}, j}^{k}\right), \quad k \geq 2 .
\end{aligned}
$$

For simplicity, we enumerate for any $k \in \mathbb{N}$ these families and call them $\left(O_{i}^{k}\right)_{i \in \mathbb{N}}$, and $\left(C_{j}^{k}\right)_{j \in \mathbb{N} \text {. }}$

Let $\bar{\Omega}:=[0,1) \times[0,1)$ and Leb be the Lebesgue measure on $[0,1) \times[0,1)$. In the first step, we will construct a family of partition consisting of rectangles in $\bar{\Omega}$.

Definition C.2 Suppose that $\mu$ is a Borel probability measure on $U=U_{1} \times U_{2}$ and $\mu^{1}$ is the marginal of $\mu$ on $U_{1}$, i.e., $\mu^{1}(O):=\mu\left(O \times U_{2}\right), O \in \mathcal{B}\left(U_{1}\right)$. Assume that $\left(O_{i}\right)_{i \in \mathbb{N}}$ and $\left(C_{i}\right)_{i \in \mathbb{N}}$ are partitions of $U_{1}$ and $U_{2}$, respectively. Define the following partition of the square $[0,1) \times[0,1)$. For $i, j \in \mathbb{N}$ we put

$$
\begin{aligned}
I_{i j}:= & {\left[\mu_{1}\left(\bigcup_{\alpha=1}^{i-1} O_{\alpha}\right), \mu_{1}\left(\bigcup_{\alpha=1}^{i} O_{\alpha}\right)\right) } \\
& \times\left[\frac{1}{\mu_{1}\left(O_{i}\right)} \mu\left(O_{i} \times \bigcup_{\alpha=1}^{j-1} C_{\alpha}\right), \frac{1}{\mu_{1}\left(O_{i}\right)} \mu\left(O_{i} \times \bigcup_{\alpha=1}^{j} C_{\alpha}\right)\right) .
\end{aligned}
$$

\footnotetext{
${ }^{7}$ For $r>0$ and $x$ let $B(x, r):=\{y,|y| \leq r\}$.
} 
Remark C.3 Obviously, if $\mu_{1}\left(O_{i}\right)=0$ for some $i \in \mathbb{N}$, then $I_{i j}=\emptyset$ for all $j \in \mathbb{N}$.

Next for fixed $l \in \mathbb{N}$ and $n \in \overline{\mathbb{N}}$, we will define a partition $\left(I_{i j}^{l, n}\right)_{i, j \in \mathbb{N}}$ of $\bar{\Omega}=$ $[0,1) \times[0,1)$ corresponding to the partitions $\left(O_{i}^{l}\right)_{i \in \mathbb{N}}$ and $\left(C_{i}^{l}\right)_{i \in \mathbb{N}}$ of the spaces $U_{1}$ and $U_{2}$, respectively.

We denote by $\mu(O \mid C)$ the conditional probability of $O$ under the condition $C$. Then, we have for $n \in \overline{\mathbb{N}}$

$$
\begin{aligned}
& I_{1,1}^{1, n}:= {\left[0, \mathcal{P} M U_{n}^{1}\left(O_{1}^{1}\right)\right) \times\left[0, \mathcal{P} M U_{n}\left(U_{1} \times C_{1}^{1} \mid O_{1}^{1} \times U_{2}\right)\right), } \\
& I_{2,1}^{1, n}:= {\left[\mathcal{P} M U_{n}^{1}\left(O_{1}^{1}\right), \mathcal{P} M U_{n}^{1}\left(O_{1}^{1}\right)+\mathcal{P} M U_{n}^{1}\left(O_{2}^{1}\right)\right) } \\
& \quad \times\left[0, \mathcal{P} M U_{n}\left(U_{1} \times C_{1}^{1} \mid O_{2}^{1} \times U_{2}\right)\right)
\end{aligned}
$$

and

$$
\begin{aligned}
I_{1,2}^{1, n} & :=\left[0, \mathcal{P} M U_{n}^{1}\left(O_{1}^{1}\right)\right) \times\left[a_{n, 1}, b_{n}\right) \\
I_{2,2}^{1, n} & :=\left[\mathcal{P} M U_{n}^{1}\left(O_{1}^{1}\right), \mathcal{P} M U_{n}^{1}\left(O_{1}^{1}\right)+\mathcal{P} M U_{n}^{1}\left(O_{2}^{1}\right)\right) \times\left[a_{n, 1}, b_{n}\right),
\end{aligned}
$$

where $a_{n, k}=\mathcal{P} M U_{n}\left(U_{1} \times C_{1}^{1} \mid O_{k}^{1} \times U_{2}\right)$ and

$$
b_{n}=\mathcal{P} M U_{n}\left(U_{1} \times C_{1}^{1} \mid O_{1}^{1} \times U_{2}\right)+\mathcal{P} M U_{n}\left(U_{1} \times C_{2}^{1} \mid O_{1}^{1} \times U_{2}\right) .
$$

More generally, for $k \in \mathbb{N}$

$$
I_{k, 2}^{1, n}:=\left[\sum_{m=1}^{k-1} \mathcal{P} M U_{n}^{1}\left(O_{k}^{1}\right), \sum_{m=1}^{k} \mathcal{P} M U_{n}^{1}\left(O_{k}^{1}\right)\right) \times\left[a_{n}, b_{n}\right),
$$

and, for $k, r \in \mathbb{N}$

$$
\begin{aligned}
& I_{k, r}^{1, n}:=\left[\sum_{m=1}^{k-1} \mathcal{P} M U_{n}^{1}\left(O_{m}^{1}\right), \sum_{m=1}^{k} \mathcal{P} M U_{n}^{1}\left(O_{m}^{1}\right)\right) \times \\
& \quad\left[\sum_{m=1}^{r-1} \mathcal{P} M U_{n}\left(U_{1} \times C_{m}^{1} \mid O_{k}^{1} \times U_{2}\right), \sum_{m=1}^{r} \mathcal{P} M U_{n}\left(U_{1} \times C_{r}^{1} \mid O_{k}^{1} \times U_{2}\right)\right) .
\end{aligned}
$$

Finally, for $k, l, r \geq 2$

$$
\begin{aligned}
I_{k, r}^{l, n}:= & {\left[\sum_{m=1}^{k-1} \mathcal{P} M U_{n}^{1}\left(O_{m}^{l}\right), \sum_{m=1}^{k} \mathcal{P} M U_{n}^{1}\left(O_{m}^{l}\right)\right) \times } \\
& {\left[\sum_{m=1}^{r-1} \mathcal{P} M U_{n}\left(U_{1} \times C_{m}^{l} \mid O_{k}^{l} \times U_{2}\right), \sum_{m=1}^{r} \mathcal{P} M U_{n}\left(U_{1} \times C_{m}^{l} \mid O_{k}^{l} \times U_{2}\right)\right) . }
\end{aligned}
$$

Let us observe that for fixed $l \in \mathbb{N}$, the rectangles $\left\{I_{k, r}^{l}: k, r \in \mathbb{N}\right\}$ are pairwise disjoint and the family $\left\{I_{k, r}^{l}: k, r \in \mathbb{N}\right\}$ is a covering of $\bar{\Omega}$. Therefore, we conclude that for any 
$n \in \mathbb{N} \cup\{\infty\}$ we have $\mathcal{P} M U_{n}\left(U_{1} \times U_{2}\right)=1$ and $\sum_{m \in \mathbb{N}} \mathcal{P} M U_{n}\left(U_{1} \times C_{m}^{l} \mid O_{k}^{l}\right)=1$. Consequently, it follows that for fixed $l, n \in \mathbb{N}$ the family of sets $\left\{I_{k, r}: k, r \in \mathbb{N}\right\}$ is a covering of $[0,1) \times[0,1)$ and consists of disjoint sets.

The next step is to construct the random variables $\bar{\chi}_{n}: \bar{\Omega} \rightarrow U_{1} \times U_{2}$, such that $\operatorname{Law}\left(\bar{\chi}_{n}\right)=\operatorname{L} a w\left(\chi_{n}\right)$. We assume that $r_{m}$ is chosen in such a way, that the measure of the boundaries of the covering $\left(O_{j}\right)_{j \in \mathbb{N}}$ and $\left(C_{j}\right)_{j \in \mathbb{N}}$ are zero. In each non-empty sets $\operatorname{int}\left(O_{j}^{m}\right)$ and $\operatorname{int}\left(C_{j}^{m}\right)$ we choose points $x_{j}^{m}$ and $y_{j}^{m}$, respectively, from the dense subsets $\left(x_{i}\right)_{i \in \mathbb{N}}$ and $\left(y_{i}\right)_{i \in \mathbb{N}}$ and define the following random variables. First, we put for $m \in \mathbb{N}$

$$
\begin{aligned}
& Z_{n, m}^{1}(\bar{\omega})=x_{k}^{m} \text { if } \bar{\omega} \in I_{k, r}^{m, n}, \\
& Z_{n, m}^{2}(\bar{\omega})=y_{r}^{m} \text { if } \bar{\omega} \in I_{k, r}^{m, n}, \quad n \in \mathbb{N} \cup\{\infty\},
\end{aligned}
$$

and then, for $n \in \mathbb{N} \cup\{\infty\}$

$$
\begin{aligned}
& \bar{\chi}_{n}^{1}(\bar{\omega})=\lim _{m \rightarrow \infty} Z_{n, m}^{1}(\bar{\omega}), \\
& \bar{\chi}_{n}^{2}(\bar{\omega})=\lim _{m \rightarrow \infty} Z_{n, m}^{2}(\bar{\omega}) .
\end{aligned}
$$

Due to the construction of the partition, the limits above exist. To be precise, for any $n \in$ $\mathbb{N} \cup\{\infty\}$ and $\bar{\omega} \in \bar{\Omega}$, we have

$$
\left|Z_{n, m}^{i}(\bar{\omega})-Z_{n, k}^{i}(\bar{\omega})\right| \leq r_{m}, \quad k \geq m, i=1,2,
$$

and therefore $\left(Z_{n, m}(\bar{\omega})\right)_{m \geq 1}$ is a Cauchy-sequence for all $\bar{\omega} \in \bar{\Omega}=[0,1) \times[0,1)$. Hence, $Z_{n}^{i}(\bar{\omega}), i, n$, is well defined. Furthermore, $\chi_{n}$ is measurable, since $Z_{n, m}^{i}$ are simple functions, hence measurable. Therefore, $Z_{n}^{i}(\bar{\omega}), i, n$, is a random variable.

Finally, we have to proof that the random variables $\bar{\chi}$ and $\bar{\chi}_{n}:=\left(\bar{\chi}_{n}{ }^{1}, \bar{\chi}_{n}{ }^{2}\right)$ have the following properties:

(i) $\mathcal{L} a w\left(\overline{\chi_{n}}\right)=\mathcal{L} a w\left(\chi_{n}\right), \forall n \in \mathbb{N}$,

(ii) $\chi_{n} \rightarrow \chi$ a.s. in $U_{1} \times U_{2}$,

(iii) $\pi_{1} \circ \chi_{n}(\omega)=\pi_{1} \circ \chi_{*}(\omega)$.

Proof of (i) The following identity holds

$$
\begin{aligned}
\operatorname{Leb}\left(I_{k, r}^{l, n}\right) & =\operatorname{Leb}\left(\overline{\chi_{n}} \in O_{k}^{l} \times C_{k}^{l}\right) \\
& =\mathcal{P} M U_{n}^{1}\left(O_{k}^{l}\right) \times \mathcal{P} M U_{n}\left(U_{1} \times C_{r}^{l} \mid O_{k}^{l} \times U_{2}\right) \\
& =\mathcal{P} M U_{n}\left(O_{k}^{l} \times U_{2}\right) \times \mathcal{P} M U_{n}\left(U_{1} \times C_{r}^{l} \mid O_{k}^{l} \times U_{2}\right) \\
& =\mathcal{P} M U_{n}\left(u^{1} \in O_{k}^{l} \text { and } u^{2} \in C_{r}^{l}\right) \\
& =\mathcal{P} M U_{1}\left(\left(u^{1}, u^{2}\right) \in O_{k}^{l} \times C_{r}^{l}\right) .
\end{aligned}
$$

Using the fact that the set of rectangles of $[0,1) \times[0,1)$ form a $\pi$-system in $\mathcal{B}([0,1) \times[0,1))$ and that Leb and $\mathcal{P} M U_{1}$ are identical on the set of rectangles, we derive from [47, Lemma 1.17, Chapter 1], Leb and $\mathcal{P} M U$ are equal on $\mathcal{B}([0,1) \times[0,1))$.

Proof of (ii) We will first prove that there exists a random variable $\chi=\left(\chi^{1}, \chi^{2}\right)$ such that ${\overline{\chi_{n}}}^{1} \rightarrow \chi^{1}$ and ${\overline{\chi_{n}}}^{2} \rightarrow \chi^{2}$ Leb-a.s. for $n \rightarrow \infty$. For this purpose it is enough to show that the sequences $\left(\bar{\chi}_{n}^{1}\right)_{n \in \mathbb{N}}$ and $\left(\bar{\chi}_{n}^{2}\right)_{n \in \mathbb{N}}$ are Leb-a.s. Cauchy sequences. From the triangle inequality we infer that for all $n, m, j \in \mathbb{N}, i=1,2$

$$
\left|\bar{\chi}_{n}^{i}-\bar{\chi}_{m}^{i}\right| \leq\left|\bar{\chi}_{n}^{i}-Z_{n, j}^{i}\right|+\left|Z_{n, j}^{i}-Z_{m, j}^{i}\right|+\left|Z_{m, j}^{i}-\bar{\chi}_{m}^{i}\right| \text {. }
$$


Let us first observe that by Eq. C.3, for any $n \in \mathbb{N}$, the sequences $\left(Z_{n, j}^{1}\right)_{j \in \mathbb{N}}$ and $\left(Z_{n, j}^{2}\right)_{j \in \mathbb{N}}$ converge uniformly on $\bar{\Omega}$ to ${\overline{\chi_{n}}}^{1}$ and $\overline{\chi_{n}}{ }^{2}$, respectively. Hence, it suffices to show that for all $\varepsilon>0$ there exists a number $n_{0}$ such that (see [34, Lemma 9.2.4])

$$
\operatorname{Leb}\left(\left\{\omega \in[0,1) \times[0,1) \mid Z_{n, l}(\omega) \neq Z_{m, l}(\Omega), n, m \geq n_{0}\right\}\right) \leq \varepsilon .
$$

Since $\left\{\mu_{n}, n \in \mathbb{N}\right\}$ converges weakly, for any $\delta>0$ there exists a number $n_{0} \in \mathbb{N}$ such that ${ }^{8}$ $\rho\left(\mu_{n}, \mu_{m}\right) \leq \varepsilon$ for all $n, m \geq n_{0}$. Hence, for any $\delta>0$ we can find a number $n_{0} \in \mathbb{N}$ such that

$$
\left|a_{k, r}^{i, l, n}-a_{k, r}^{i, l, m}\right|,\left|b_{k, r}^{i, l, n}-b_{k, r}^{i, l, m}\right| \leq \delta, \quad k, r, l \in \mathbb{N}, i=1,2, n, m \geq n_{0},
$$

where

$$
I_{k, r}^{l, n}=\left[a_{k, r}^{1, l, n}, b_{k, r}^{1, l, n}\right) \times\left[a_{k, r}^{2, l, n}, b_{k, r}^{2, l, n}\right),
$$

and

$$
I_{k, r}^{l, m}=\left[a_{k, r}^{1, l, m}, b_{k, r}^{1, l, m}\right) \times\left[a_{k, r}^{2, l, m}, b_{k, r}^{2, l, m}\right) .
$$

In fact, by the construction of $I_{k, r}^{l, n}$ and $I_{k, r}^{l}$, we have

$$
\begin{aligned}
& a_{k, r}^{1, l, n}=\mu_{n}^{1}\left(\cup_{j=1}^{k-1} O_{j}^{l}\right), n, m \in \mathbb{N}, r, k \in \mathbb{N}, \\
& a_{k, r}^{1, l, m}=\mu_{m}^{1}\left(\cup_{j=1}^{k-1} O_{j}^{l}\right), n, m \in \mathbb{N}, r, k \in \mathbb{N} .
\end{aligned}
$$

Let us now fix $\delta>0$ and let us choose $n_{0}$ such that $\rho\left(\mu_{n}, \mu_{m}\right) \leq \delta, n, m \geq n_{0}$. Then,

$$
\mu_{n}^{1}\left(\cup_{j=1}^{k-1} O_{j}^{l}\right) \leq \mu_{n}^{1}\left(\left(\cup_{j=1}^{k-1} O_{j}^{l}\right)^{\delta}\right) \leq \mu_{m}^{1}\left(\cup_{j=1}^{k-1} O_{j}^{l}\right)+\delta, \quad r, k, l \in \mathbb{N} .
$$

On the other hand, by symmetricity of the Prokhorov metric,

$$
\mu_{m}^{1}\left(\cup_{j=1}^{k-1} O_{j}^{l}\right) \leq \mu_{m}^{1}\left(\left(\cup_{j=1}^{k-1} O_{j}^{l}\right)^{\delta}\right) \leq \mu_{n}^{1}\left(\cup_{j=1}^{k-1} O_{j}^{l}\right)+\delta, \quad r, k \in \mathbb{N} .
$$

Hence, we infer that

$$
\left|a_{k, r}^{1, l, n}-a_{k, r}^{1, l, m}\right| \leq \delta, \quad r, k, l \in \mathbb{N}, \quad n, m \geq n_{0} .
$$

The second inequality in Eq. C.4 can be proved in a similar way.

Since the sequence $\left(\mu_{n}\right)_{n \in \mathbb{N}}$ is tight on $U_{1} \times U_{2}$, we can find a compact set $K_{1} \times K_{2}$ such that

$$
\sup _{n}\left(\mu_{n}\left(\left(U_{1} \times U_{2}\right) \backslash\left(K_{1} \times K_{2}\right)\right)\right) \leq \frac{\varepsilon}{2} .
$$

Let us fix $l \in \mathbb{N}$. Since the set $K_{1} \times K_{2}$ is compact, from the covering $\left(O_{k}^{l} \times C_{r}^{l}\right)_{k \in \mathbb{N}, r \in \mathbb{N}}$ of $U_{1} \times U_{2}$ there exists a finite covering $\left(O_{k}^{l} \times C_{r}^{l}\right)_{k=1, \cdots, K, j=1, \cdots, R}$ of $K_{1} \times K_{2}$. Next, observe that the estimate (C.4) is uniformly for all $n, m \geq n_{0}$. Therefore we can use estimate (C.4) with $\delta=\varepsilon / 2(K R)$ and infer that

$$
\sum_{k, r=1}^{K, R} \operatorname{Leb}\left(I_{k, r}^{l, n} \triangle I_{k, r}^{l, m}, n, m \geq n_{0}\right) \leq \frac{\varepsilon}{2} .
$$

${ }^{8} \rho$ denotes the Prokhorov metric on measurable space $(U, \mathcal{U})$, i.e., $\rho(F, G):=\inf \left\{\varepsilon>0: F\left(O^{\varepsilon}\right) \leq\right.$ $G(O)+\varepsilon, O \in \mathcal{U}\}$. 
Moreover, since

$$
\begin{aligned}
& \operatorname{Leb}\left(\left\{\omega \in[0,1) \times[0,1): Z_{n, l}(\omega) \neq Z_{m, l}(\omega), n, m \geq n_{0}\right\}\right) \leq \\
& \quad \leq \sum_{k, r=1} \operatorname{Leb}\left(I_{k, r}^{l, n} \Delta I_{k, r}^{l, m}, n, m \geq n_{0}\right)+\operatorname{Leb}\left(\left(U_{1} \times U_{2}\right) \backslash\left(K_{1} \times K_{2}\right)\right),
\end{aligned}
$$

it follows that

$$
\operatorname{Leb}\left(\left\{\omega \in[0,1) \times[0,1): Z_{n, l}(\omega) \neq Z_{m, l}(\omega), n, m \geq n_{0}\right\}\right) \leq \frac{\varepsilon}{2}+\frac{\varepsilon}{2} .
$$

Summarizing, we proved that for any $\varepsilon>0$ there exists a number $n_{0} \in \mathbb{N}$ such that $\left\{\omega \in[0,1) \times[0,1) \mid Z_{n, l}(\omega) \neq Z_{m, l}(\Omega), n, m \geq n_{0}\right\} \leq \varepsilon$. From [34, Lemma 9.2.4] we infer (ii).

Proof of (iii) Let us denote

$$
J_{k}^{l, n}:=\left[\sum_{m=1}^{k-1} \mathcal{P} M U_{n}^{1}\left(O_{k}^{l}\right), \sum_{m=1}^{k} \mathcal{P} M U_{n}^{1}\left(O_{k}^{l}\right)\right), \quad k=1, \cdots, N_{l}^{1} .
$$

Since the laws of $\pi_{1} \circ \chi_{n}$ and $\pi_{1} \circ \chi_{n}, n, m \in \overline{\mathbb{N}}$ are equal, $J_{k}^{l, n}=J_{k}^{l, m}, n, m \in \overline{\mathbb{N}}$. Let us denote these (equal) sets $J_{k}^{l}$. Since for each $J_{k}^{l}$ we can find a set $O_{k}^{l}$ satisfying (C.6) for all $n \in \overline{\mathbb{N}}$, we infer that for any $m \in \mathbb{N}$,

$$
Z_{n, m}^{1}(\bar{\omega})=Z_{1, m}^{1}(\bar{\omega}), \quad \bar{\omega} \in \Omega, n \in \overline{\mathbb{N}} .
$$

Let $n \in \mathbb{N}$ be fixed. Considering the limit of the sequence $\left(Z_{n, m}^{1}(\bar{\omega})\right)_{m \in \mathbb{N}}$ as $m \rightarrow \infty$ and keeping in mind that $\left(Z_{n, m}^{1}(\bar{\omega})\right)_{m \in \mathbb{N}}$ is a Cauchy sequence implies the assertion (iii).

\section{Appendix D: A Tightness Criteria in $\mathbb{D}([0, T] ; Y)$}

Let $Y$ be a separable and complete metric space and $T>0$. The space $\mathbb{D}([0, T] ; Y)$ denotes the space of all right continuous functions $x:[0, T] \rightarrow Y$ with left limits. The space of continuous function is usually equipped with the uniform topology. But, since $\mathbb{D}([0, T] ; Y)$ is complete but not separable in the uniform topology, we equip it with the $J_{1}$-Skorohod topology, i.e., the finest among all Skorohod topologies, with which $\mathbb{D}([0, T] ; Y)$ is both separable and complete. For more information about Skorohod space and its topology we refer to Billingsley's book [5] or Ethier and Kurtz [36]. In this appendix we only state the following tightness criterion which is necessary for our work.

Theorem D.1 ${ }^{9}$ A subset $C$ of the space $\mathcal{P}(\mathbb{D}([0, T] ; Y))$ of all Borel probability measures on $\mathbb{D}([0, T] ; Y)$ is tight, iff

a.) for any $\varepsilon>0$ there exists a compact set $K \subset Y$ such that for every $F \in C$

$$
F(\{x \in \mathbb{D}([0, T] ; Y): x(t) \in K \forall t \in[0, T]\}) \geq 1-\varepsilon ;
$$

\footnotetext{
${ }^{9}$ Compare with [5, Chapter III, Theorem 13.5, p. 142].
} 
b.) there exist two real numbers $\gamma>0, c>0$ and a nondecreasing continuous function $g:[0, T] \rightarrow \mathbb{R}_{+}$such that for all $t_{1} \leq t \leq t_{2}, n \geq 0$ and $\lambda>0$

$$
\begin{gathered}
F\left(\left\{x \in \mathbb{D}([0, T] ; Y):\left|x(t)-x\left(t_{1}\right)\right| \geq \lambda,\left|x(t)-x\left(t_{2}\right)\right| \geq \lambda\right\}\right) \\
\leq \frac{c}{\lambda^{2 \gamma}}\left[g\left(t_{2}\right)-g\left(t_{1}\right)\right], \quad \forall F \in C .
\end{gathered}
$$

Corollary D.2 Let $\left\{x_{n}: n \in \mathbb{N}\right\}$ be a sequence of $Y$-valued càdlàg processes, each of the process defined on a probability space $\left(\Omega_{n}, \mathcal{F}_{n}, \mathbb{P}_{n}\right)$. Then the sequence of the laws of $\left\{x_{n}: n \in \mathbb{N}\right\}$ is tight on $\mathbb{D}([0, T] ; Y)$ if

(a) for any $\varepsilon>0$ there exists a compact set $K_{\varepsilon} \subset Y$ such that

$$
\mathbb{P}_{n}\left(x_{n}(t) \in K_{\varepsilon}, t \in[0, T]\right) \geq 1-\varepsilon, \forall n \in \mathbb{N} ;
$$

(b) there exist two constants $c>0$ and $\gamma>0$ and a real number $r>0$ such that for all $\theta>0, t \in[0, T-\theta]$, and $n \geq 0$

$$
\mathbb{E}_{n} \sup _{t \leq s \leq t+\theta}\left|x_{n}(t)-x_{n}(s)\right|^{r} \leq c \theta^{\gamma}
$$

Proof Corollary D.2-(a) and the Chebyshev inequality imply Theorem D.1-(a). Now fix $t_{1} \leq t \leq t_{2}$. Then

$$
\begin{aligned}
& \mathbb{P}_{n}\left(\left|x_{n}(t)-x_{n}\left(t_{1}\right)\right| \geq \lambda,\left|x_{n}(t)-x_{n}\left(t_{2}\right)\right| \geq \lambda\right) \\
& \quad \leq \mathbb{P}_{n}\left(\sup _{t_{1} \leq s \leq t_{2}}\left|x_{n}(s)-x_{n}\left(t_{1}\right)\right| \geq \lambda\right) .
\end{aligned}
$$

Estimating the RHS by the Chebyshev inequality and using Corollary D.2-(b) leads to Theorem D.1-(b). This completes the proof of the corollary.

\section{Appendix E: An Inequality}

Let $Y$ be a Banach space with norm $|\cdot|, T>0$ and $f:(0, T] \rightarrow Y$ is a Bochner integrable function such that

$$
\int_{0}^{T}|f(s)|^{p} d s<\infty .
$$

For fixed $n$ let $I_{k}=\left(\frac{k}{2^{n}}, \frac{k+1}{2^{n}}\right]$ and $\hat{f}_{n}:(0, T] \rightarrow Y$ be the function defined by $\hat{f}_{n}(s)=0$ for $s \in I_{0}$ and $\hat{f}_{n}(s)=2^{n} \int_{I_{k}} f(t) d t$ for $s \in I_{k+1}, k=0,1,2, \cdots$. We have the following facts.

Proposition E.1 (i) If $f$ belongs to $L^{p}(0, T ; Y)$, then $\hat{f}_{n} \in L^{p}(0, T ; Y)$ for each $n$. (ii) Let $\alpha \in\left(0, \frac{1}{p}\right)$. Then, there exists $C>0$ such that for all $f \in W^{\alpha, p}(0, T ; Y)$ and $n$

$$
\left\|f(s)-\hat{f}_{n}(s)\right\|_{L^{p}(0, T ; Y)} \leq C 2^{-n \alpha}\|f\|_{W^{p, \alpha}(0, T ; Y)} .
$$


Proof Without loss of generality we take $T=1$ and set $t_{j}^{n}=\frac{j}{2^{n}}$. We have

$$
\begin{aligned}
\int_{0}^{1}\left|\hat{f}_{n}(s)\right|^{p} d s & =\sum_{j=1}^{2^{n}-1} \int_{t_{j}^{n}}^{t_{j+1}^{n}}\left|\hat{f}_{n}(s)\right|^{p} d s \\
& =\sum_{j=1}^{2^{n}-1} \frac{1}{2^{n}}\left|\hat{f}_{n}\left(t_{j}^{n}\right)\right|^{p} .
\end{aligned}
$$

From this last equation and the definition of $\hat{f}$ we derive

$$
\begin{aligned}
\int_{0}^{1}\left|\hat{f}_{n}(s)\right|^{p} d s & \leq \sum_{j=1}^{2^{n}-1} \frac{1}{2^{n}}\left|2^{n} \int_{t_{j-1}^{n}}^{t_{j}^{n}} f(s) d s\right|^{p} \\
& \leq \sum_{j=1}^{2^{n}-1} \int_{t_{j-1}^{n}}^{t_{j}^{n}}|f(s)|^{p} d s .
\end{aligned}
$$

Therefore

$$
\int_{0}^{1}\left|\hat{f}_{n}(s)\right|^{p} d s \leq \int_{0}^{1-\frac{1}{2^{n}}}|f(s)|^{p} d s,
$$

which ends the proof of (i).

Next we will prove item (ii). Let $s \in[0,1], \alpha \in\left(0, \frac{1}{p}\right)$ and $f \in W^{\alpha, p}(0, T ; Y)$. Since the intervals $I_{k}, k=0,1, \ldots, 2^{n}-1$, form a partition of $[0,1]$ then either $s \in I_{0}$ or $s \in\left(2^{-n}, 1\right]$. In one hand if $s \in\left(2^{-n}, 1\right]$ then there exists $k \geq 1$ such that $s \in I_{k}$. In this case we have by Hölder's inequality that

$$
\begin{array}{r}
\left|f(s)-\hat{f}_{n}(s)\right| \leq 2^{n} \mid \int_{I_{k-1}} \frac{(f(s)-f(r))}{|s-r|^{\frac{1}{p}+\alpha} \times|s-r|^{\frac{1}{p}+\alpha} d r \mid} \\
\leq 2^{n}\left[\int_{I_{k-1}}|s-r|^{\frac{p}{p-1}(1+\alpha)-1} d r\right]^{\frac{p-1}{p}}\left[\int_{I_{k-1}} \frac{|f(s)-f(r)|^{p}}{|s-r|^{1+p \alpha}} d r\right]^{\frac{1}{p}} \\
\leq 2^{-n p \alpha}\left[\int_{I_{k-1}} \frac{|f(s)-f(r)|^{p}}{|s-r|^{1+p \alpha}} d r\right]^{\frac{1}{p}} .
\end{array}
$$

Therefore

$$
\begin{gathered}
\int_{2^{-n}}^{1}\left|f(s)-\hat{f}_{n}(s)\right|^{p} d s \leq 2^{-n p \alpha} \int_{2^{-n}}^{1}\left[\sum_{k=1}^{2^{n}-1} \int_{I_{k-1}} \frac{|f(s)-f(r)|^{p}}{|s-r|^{1+p \alpha}} d r\right] d s \\
\leq 2^{-n p \alpha} \int_{0}^{1} \int_{0}^{1} \frac{|f(s)-f(r)|^{p}}{|s-r|^{1+p \alpha}} d r d s \leq 2^{-n p \alpha}\|f\|_{W^{\alpha, p}(0, T ; Y)}^{p} .
\end{gathered}
$$

On the other hand, by making use of the Hölder Inequality we obtain

$$
\begin{aligned}
\int_{0}^{2^{-n}}\left|f(s)-\hat{f}_{n}(s)\right|^{p} d s & =\int_{0}^{2^{-n}}|f(s)|^{p} d s \\
& \leq 2^{-n p \alpha}\left[\int_{0}^{2^{-n}}|f(s)|^{q} d s\right]^{\frac{p}{q}},
\end{aligned}
$$


where $q=\frac{p}{1-p \alpha}$. Since $W^{\alpha, p}\left(I_{0}\right) \subset L^{r}\left(I_{0}\right)$ for any $r \in\left[1, \frac{p}{1-p \alpha}\right]$ we infer from the last inequality that there exists $C>0$ such that

$$
\int_{0}^{2^{-n}}\left|f(s)-\hat{f}_{n}(s)\right|^{p} d s \leq C 2^{-n p \alpha}\|f\|_{W^{\alpha, p}(0, T ; Y)}^{p} .
$$

Now inequality (E.1) follows from inequalities (E.2) and (E.3). This completes the proof of our proposition.

\section{References}

1. Applebaum, D.: Lévy processes and stochastic integrals in Banach spaces. Probab. Math. Statist 27(1), 77-88 (2007)

2. Applebaum, D., Riedle, M.: Cylindrical Lévy Processes in Banach Spaces. Proc. Lond. Math. Soc. (3) 101, 697-726 (2010)

3. Bandini, E., Russo, F.: Special weak Dirichlet processes and BSDEs driven by a random measure. arXiv:1512.06234v2 (2015)

4. Barbu, V.: Private Communication. Innsbruck (2011)

5. Billingsley, P.: Convergence of Probability Measures, 2nd edn. Wiley Series in Probability and Statistics. Probability and Statistics. A Wiley-Interscience Publication. Wiley, New York (1999)

6. Bergh, J., Löfström, J.: Interpolation Spaces: An Introduction, Volume 223 of Die Grundlehren der mathematischen Wissenschaften. Springer Verlag, Berlin (1976)

7. Saint Loubert Bié, E.: Étude d'une EDPS conduite par un bruit poissonnien. (Study of a stochastic partial differential equation driven by a Poisson noise). Probab. Theory Relat. Fields 111, 287-321 (1998)

8. Brézis, H., Friedman, A.: Nonlinear parabolic equations involving measures as initial conditions. J. Math. Pures Appl. (9) 62, 173-97 (1983)

9. Brzeźniak, Z.: Stochastic partial differential equations in M-type 2 Banach spaces. Potential Anal. 4, $1-45$ (1995)

10. Brzeźniak, Z., Gảtarek, D.: Martingale solutions and invariant measures for stochastic evolution equations in Banach spaces. Stoch. Process Appl. 84, 187-225 (1999)

11. Brzeźniak, Z., Goldys, B., Imkeller, P., Peszat, S., Priola, E., Zabczyk, J.: Time irregularity of generalized Ornstein-Uhlenbeck processes. C. R. Math. Acad. Sci. Paris 348(5-6), 273-276 (2010)

12. Brzeźniak, Z., Peszat, S.: Stochastic two dimensional Euler equations. Ann. Probab. 29, 1796-1832 (2001)

13. Brzeźniak, Z., Hausenblas, E.: Maximal regularity of stochastic convolution with Lévy noise. Probab. Theory Related Fields 145, 615-637 (2009)

14. Brzeźniak, Z., Hausenblas, E.: Uniqueness in Law of the Iô integral driven by Lévy noise. In: Proceedings of the Workshop Stochastic Analysis, Random Fields and Applications - VI, Ascona, Switzerland Progr. Probab, vol. 63, p. 2011. Birkhäuser/Springer Basel AG, Basel (2008)

15. Brzeźniak, Z., Hausenblas, E., Motyl, E.: Uniqueness in Law of the stochastic convolution driven by Lévy noise. Electron. J. Probab. 18, 1-15 (2013)

16. Brzeźniak, Z., Hausenblas, E., Razafimandimby, P.: A note on the Lévy-Itô decomposition in Banach spaces (in preparation)

17. Brzeźniak, Z., Hausenblas, E., Zhu, J.: 2D stochastic Navier-Stokes equations driven by jump noise. Nonlinear Anal. 79, 122-139 (2013)

18. Brzezńiak, Z., Ondreját, M.: Stochastic geometric wave equations with values in compact Riemannian homogeneous spaces. Ann. Probab. 41, 1938-1977 (2013)

19. Brzeźniak, Z., Zabczyk, J.: Regularity of Ornstein-Uhlenbeck processes driven by a Lévy white noise. Potential Anal. 32, 153-188 (2010)

20. Cerrai, S.: Stochastic reaction-diffusion systems with multiplicative noise and non-Lipschitz reaction term. Probab. Theory Related Fields 125(2), 271-304 (2003)

21. Chalk, C.: Nonlinear evolutionary equations in Banach spaces with fractional time derivative. $\mathrm{PhD}$ thesis, The University of Hull, Kingston upon Hull, UK (2006)

22. Dalang, R.: Level sets and excursions of the Brownian sheet. Topics in spatial stochastic processes (Martina Franca, 2001), 167208, Lecture Notes in Math, vol. 1802. Springer, Berlin (2003) 
23. Da Prato, G.: Applications croissantes et équations d'évolution dans les espaces de Banach. Academic Press, London (1976)

24. Da Prato, G., Zabczyk, J.: Stochastic Equations in Infinite Dimensions, Volume 44 of Encyclopedia of Mathematics and Its Applications. Cambridge University Press, Cambridge (1992)

25. Debussche, A., Högele, M., Imkeller, P.: Asymptotic first exit times of the Chafee-Infante equation with small heavy-tailed Lévy noise. Electron. Commun. Probab. 16, 213-225 (2011)

26. Debussche, A., Högele, M., Imkeller, P.: The Dynamics of Nonlinear Reaction-Diffusion Equations with Small Lévy noise. Lecture Notes in Mathematics, vol. 2085. Springer, Berlin (2013)

27. Dettweiler, E.: Representation of Banach space valued martingales as stochastic integrals. In: Probability in Banach Spaces, 7 (Oberwolfach, 1988) Progr. Probab., vol. 21, pp. 43-62 (1990)

28. Di Blasio, G.: Linear parabolic evolution equations in $L^{p}$-spaces. Ann. Mat. Pura Appl. (4) 138, 55-104 (1984)

29. Diestel, J., Uhl, J.J., Jr.: Vector measures, with a Foreword by B. J. Pettis Mathematical Surveys, vol. 15. American Mathematical Society, Providence (1977)

30. Dirksen, S.: Itô isomorphisms for $L^{p}$-valued Poisson stochastic integrals. Ann. Probab. 42(6), 25952643 (2014)

31. Dong, Z., Xu, T.G.: One-dimensional stochastic Burgers' equations driven by Lévy processes. J. Funct. Anal. 243, 631-678 (2007)

32. Dong, Z., Xie, Y.: Global solutions of stochastic 2D Navier-Stokes equations with Lèvy noise. Sci. China Ser. A 52(7), 1497-1524 (2009)

33. Dore, G., Venni, A.: On the closedness of the sum of two closed operators. Math. Z. 196, 189-201 (1987)

34. Dudley, R.: Real Analysis and Probability. Cambridge Studies in Advanced Mathematics, vol. 74. Cambridge University Press, Cambridge (2002)

35. Engel, K.-J., Nagel, R.: One-parameter semigroups for linear evolution equations. In: Graduate Texts in Mathematics, vol. 194. Springer-Verlag, New York (2000)

36. Ethier, S., Kurtz, T.: Markov Processes, Characterization and Convergence. Wiley Series in Probability and Mathematical Statistics: Probability and Mathematical Statistics. Wiley, New York (1986)

37. Fernando, B.P.W., Sritharan, S.: Nonlinear Filtering of Stochastic Navier-Stokes Equation with ItoLévy Noise. Stoch. Anal. Appl. 31(3), 381-426 (2013)

38. Friedman, A.: Partial Differential Equations. Holt, Rinehart and Winston, Inc., New York (1969)

39. Giga, Y., Sohr, H.: Abstract $L p$ estimates for the Cauchy problem with applications to the Navier-Stokes equations in exterior domains. J. Funct. Anal. 102, 72-94 (1991)

40. Flandoli, F., Ga̧tarek, D.: Martingale and stationary solutions for stochastic Navier-Stokes equations. Probab. Theory Related Fields 102, 367-391 (1995)

41. Hausenblas, E., Giri, A.: Stochastic Burgers equation with polynomial nonlinearity driven by Lévy process. Commun. Stoch. Anal. 7, 91-112 (2013)

42. Hausenblas, E.: Existence, uniqueness and regularity of parabolic SPDEs driven by poisson random measure. Electron. J. Probab. 10, 1496-1546 (2005)

43. Hausenblas, E.: SPDEs driven by Poisson Random measure with non Lipschitz coefficients. Probab. Theory Related Fields 137, 161-200 (2007)

44. Hausenblas, E., Razafimandimby, P., Sango, M.: Martingale solution to equations for differential type fluids of grade two driven by random force of Lévy type. Potential Anal. 38(04), 1291-1331 (2013)

45. Ikeda, N., Watanabe, S.: Stochastic Differential Equations and Diffusion Processes, Volume 24 of North-Holland Mathematical Library, 2nd ed. North-Holland Publishing Co., Amsterdam (1989)

46. Jacod, J., Shiryaev, A.: Limit theorems for stochastic processes. In: Grundlehren der Mathematischen Wissenschaften [Fundamental Principles of Mathematical Sciences]. 2nd edn., vol. 288. Springer-Verlag, Berlin (2003)

47. Kallenberg, O.: Foundations of Modern Probability. Probability and its Applications (New York), 2nd edn. Springer-Verlag, New York (2002)

48. Kotelenez, P.: A submartingale type inequality with applications to stochastic evolution equations. Stochastics 8(2), 139-151 (1982/83)

49. Kahane, J.P.: Some Random Series of Functions, 2nd edn. Cambridge University Press, Cambridge (1985)

50. Kwapień, S., Woyczyński, W.A.: Random Series and Stochastic Integrals: Single and Multiple. Birkhäuser, Boston (1992)

51. Linde, W.: Probability in Banach Spaces - Stable and Infinitely Divisible Distributions, 2nd edn. A Wiley-Interscience Publication (1986)

52. Lions, J.L., Magenes, E.: Non-homogeneous Boundary Value Problems and Applications. Vol. I, Grundlehren der Mathematischen Wissenschaften [Fundamental Principles of Mathematical Sciences], p. 181. Springer-Verlag, New York (1972) 
53. Lunardi, A.: Analytic Semigroups and Optimal Regularity in Parabolic Problems. [2013 reprint of the 1995 original]. Modern Birkhäuser Classics. Birkhäuser/Springer, Basel (1995)

54. Mandrekar, V., Rüdiger, B.: Existence and uniqueness of path wise solutions for stochastic integral equations driven by Lévy noise on separable Banach spaces. Stochastics 78, 189-212 (2006)

55. Marinelli, C., Röckner, M.: Well-posedness and asymptotic behavior for stochastic reaction-diffusion equations with multiplicative Poisson noise. Electron. J. Probab. 15, 1528-1555 (2010)

56. Metivier, M.: Semimartingales: A Course on Stochastic Processes. de Gruyter, Berlin (1982)

57. Motyl, E.: Stochastic Navier-Stokes equations driven by Lévy noise in unbounded 3D domains. Potential Anal. 38, 863-912 (2013)

58. Mueller, C.: The heat equation with Lévy noise. Stoch. Process Appl. 74, 67-82 (1998)

59. Mueller, C., Mytnik, L., Stan, A.: The heat equation with time-independent multiplicative stable Lévy noise. Stoch. Process Appl. 116, 70-100 (2006)

60. Mytnik, L.: Stochastic partial differential equation driven by stable noise. Probab. Theory Related Fields 123, 157-201 (2002)

61. Jacob, N., Potrykus, A., Wu, J.-L.: Solving a non-linear stochastic pseudo-differential equation of Burgers type. Stoch. Process Appl. 120, 2447-2467 (2010)

62. Parthasarathy, K.R.: Probability measures on metric spaces. Academic Press, New York (1967)

63. Pazy, A.: Semigroups of Linear Operators and Applications to Partial Differential Equations, Volume 44 of Applied Mathematical Sciences. Springer-Verlag, New York (1983)

64. Peszat, S.: Stochastic partial differential equations with Lévy noise (a few aspects). In: Dalang, R., et al. (eds.) Stochastic Analysis: A Series of Lectures, Centre Interfacultaire Bernoulli, January-June 2012, Progress in Probability 68, pp. 333-357. Springer, Berlin (2015)

65. Peszat, S., Zabczyk, J.: Stochastic Partial Differential Equations with Lévy Noise, Volume 113 of Encyclopedia of Mathematics and its Applications. Cambridge University Press, Cambridge (2007)

66. Peszat, S., Zabczyk, J.: Time regularity of solutions to linear equations with Lévy noise in infinite dimensions. Stoch. Process Appl. 123, 719-751 (2013)

67. Röckner, M., Zhang, T.: Stochastic evolution equations of jump type: existence, uniqueness and large deviation principles. Potential Anal. 26, 255-279 (2007)

68. Reed, M., Simon, B.: Methods of Modern Mathematical Physics. II. Fourier Analysis, Self-adjointness. Academic Press [Harcourt Brace Jovanovich Publishers], New York (1975)

69. Runst, T., Sickel, W.: Sobolev Spaces of Fractional Order, Nemytskii Operators and Nonlinear Partial Differential Equations., Volume 3 of de Gruyter Series in Nonlinear Analysis and Applications. de Gruyter, Berlin (1996)

70. Sato, K.-I.: Lévy Processes and Infinite Divisible Distributions. Cambridge Studies in Advanced Mathematics, vol. 68. Cambridge University Press, Cambridge (2005)

71. Seeley, R.: Norms and domains of the complex powers $A_{B}^{z}$. Amer. J. Math. 93, 299-309 (1971)

72. Triebel, H.: Interpolation Theory, Function Spaces, Differential Operators, North-Holland Mathematical Library, vol. 18. North-Holland Publishing Co., Amsterdam-New York (1978)

73. Triebel, H.: Interpolation Theory, Function Spaces, Differential Operators. 2nd rev. a. enl ed. Barth, Leipzig (1995)

74. Truman, A., Wu, J.-J.: Fractal Burgers' Equation Driven by Lévy Noise. Stochastic Partial Differential Equations and Applications-VII, Volume 245 Lect. Notes Pure Appl. Math., pp. 295-310. Chapman \& Hall/CRC, Boca Raton (2006)

75. Truman, A., Wu, J.-L.: Stochastic Burgers' Equation with Lévy Space-time White Noise. Probabilistic Methods in Fluids, vol. 298-323. World Sci. Publ., River Edge (2003)

76. Whitt, W.: Some useful functions for functional limit theorems. Math. Oper. Res. 5, 67-85 (1980)

77. Zhu, J., Brzeźniak, Z., Hausenblas, E.: Maximal inequality of stochastic convolution driven by compensated Poisson random measures in Banach spaces. Ann. Inst. H. Poincaré Probab. Statist. 53(2), 937-956 (2017). https://doi.org/10.1214/16-AIHP743 